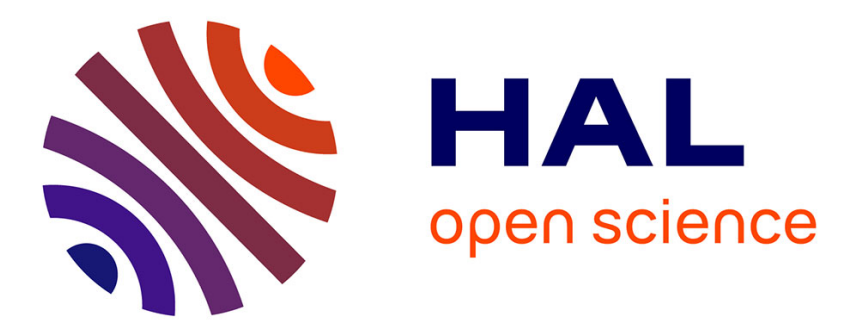

\title{
Partial regularity for fractional harmonic maps into spheres
}

Vincent Millot, Marc Pegon, Armin Schikorra

\section{To cite this version:}

Vincent Millot, Marc Pegon, Armin Schikorra. Partial regularity for fractional harmonic maps into spheres. Archive for Rational Mechanics and Analysis, 2021, 242 (2), pp.747-825. 10.1007/s00205021-01693-w . hal-02297231v3

\section{HAL Id: hal-02297231 \\ https://hal.science/hal-02297231v3}

Submitted on 12 Oct 2020

HAL is a multi-disciplinary open access archive for the deposit and dissemination of scientific research documents, whether they are published or not. The documents may come from teaching and research institutions in France or abroad, or from public or private research centers.
L'archive ouverte pluridisciplinaire HAL, est destinée au dépôt et à la diffusion de documents scientifiques de niveau recherche, publiés ou non, émanant des établissements d'enseignement et de recherche français ou étrangers, des laboratoires publics ou privés. 


\title{
PARTIAL REGULARITY FOR FRACTIONAL HARMONIC MAPS INTO SPHERES
}

\author{
VINCENT MILLOT, MARC PEGON, AND ARMIN SCHIKORRA
}

\begin{abstract}
This article addresses the regularity issue for stationary or minimizing fractional harmonic maps into spheres of order $s \in(0,1)$ in arbitrary dimensions. It is shown that such fractional harmonic maps are $C^{\infty}$ away from a small closed singular set. The Hausdorff dimension of the singular set is also estimated in terms of $s \in(0,1)$ and the stationarity/minimality assumption.
\end{abstract}

\section{CONTEnts}

1. Introduction

2. Functional spaces, fractional operators, and compensated compactness

3. Fractional harmonic maps and weighted harmonic maps with free boundary $\quad 15$

4. Small energy Hölder regularity

5. Small energy Lipschitz regularity

6. Higher order regularity

7. Partial regularity for stationary and minimizing $s$-harmonic maps 38

Appendix A. On the degenerate Laplace equation $\quad 49$

$\begin{array}{lll}\text { Appendix B. A Lipschitz estimate for } s \text {-harmonic functions } & 50\end{array}$

Appendix C. An embedding theorem between generalized $\mathcal{Q}_{\alpha}$-spaces 51

References

\section{INTRODUCTION}

The theory of fractional harmonic maps into a manifold is quite recent. It is has been initiated some years ago by F. Da Lio and T. Rivière in $[9,10]$. In those first articles, they have introduced and studied 1/2-harmonic maps from the real line into a smooth and compact closed submanifold $\mathcal{N} \subseteq \mathbb{R}^{d}$. A map $u: \mathbb{R} \rightarrow \mathcal{N}$ is said to be a 1/2-harmonic map into $\mathcal{N}$ if it is a critical point of the $1 / 2$-Dirichlet energy

$$
\mathcal{E}_{\frac{1}{2}}(u, \mathbb{R}):=\frac{1}{2} \int_{\mathbb{R}}\left|(-\Delta)^{\frac{1}{4}} u\right|^{2} \mathrm{~d} x=\frac{1}{4 \pi} \iint_{\mathbb{R} \times \mathbb{R}} \frac{|u(x)-u(y)|^{2}}{|x-y|^{2}} \mathrm{~d} x \mathrm{~d} y,
$$

among all maps with values into $\mathcal{N}$, or equivalently, if it satisfies the Euler-Lagrange equation

$$
(-\Delta)^{\frac{1}{2}} u \perp \operatorname{Tan}(u, \mathcal{N})
$$

in the distributional sense. Here $(-\Delta)^{s}$ denotes the integro-differential (multiplier) operator associated to the Fourier symbol $(2 \pi|\xi|)^{2 s}, s \in(0,1)$. The notion of $1 / 2$-harmonic map into $\mathcal{N}$ appears in several geometrical problems, such as free boundary minimal surfaces or Steklov eigenvalue problems, see [8] and references therein. The special case $\mathcal{N}=\mathbb{S}^{d-1}$ is important for both geometrical and analytical issues. From the analytical point of view, it enlightens the internal structure of equation (1.1). Indeed, the Lagrange multiplier associated to the constraint to be $\mathbb{S}^{d-1}$-valued takes a very simple form, and (1.1) reduces to the equation

$$
(-\Delta)^{\frac{1}{2}} u(x)=\left(\frac{1}{2 \pi} \int_{\mathbb{R}} \frac{|u(x)-u(y)|^{2}}{|x-y|^{2}} \mathrm{~d} y\right) u(x),
$$

which is in clear analogy with the equation for usual harmonic maps from a $2 \mathrm{~d}$-domain into the sphere. In particular, there is a similar analytical issue concerning regularity of solutions since the right hand side of (1.2) has a priori no better integrability than $L^{1}$, and elliptic linear theory does not apply. In their pioneering work [9], F. Da Lio and T. Rivière proved 
complete smoothness of 1/2-harmonic maps through a reformulation of equation (1.2) in terms of algebraic quantities, the "3-terms commutators", exhibiting some compensation phenomena. In [10] (dealing with arbitrary targets), smoothness of 1/2-harmonic maps follows from a more general compensation result for nonlocal systems with antisymmetric potential, in the spirit of [39]. In the same stream of ideas, K. Mazowiecka and the third author obtained in [29] a new proof of the regularity of $1 / 2$-harmonic maps, very close to the original argument of F. Hélein [24] to prove smoothness of harmonic maps from surfaces into spheres (see also [25]). Once again, the key point in [29] is to rewrite the right hand side of (1.2) to discover a suitable "fractional div-curl structure". From the new form of the equation, they deduce that $(-\Delta)^{\frac{1}{2}} u$ belongs (essentially) to the Hardy space $\mathcal{H}^{1}$ by applying their main result [29, Theorem 2.1], a generalization to the fractional setting of the div-curl estimate of R. Coifman, P.L. Lions, Y. Meyer, and S. Semmes [4]. Continuity of solutions is then a consequence of CalderónZygmund theory, from which it is possible to deduce $C^{\infty}$-regularity.

Several generalizations of the regularity result of $[9,10]$ have been obtained, e.g. for critical points of higher order or/and $p$-power type energies (still in the corresponding critical dimension), see $[7,11,12,29,44,45,46]$. The regularity theory for $1 / 2$-harmonic maps into a manifold in higher dimensions has been addressed in [36] and [32] (see also [30]). In higher dimensions, the theory provides partial regularity (i.e. regularity away from a "small" singular set) for stationary 1/2-harmonic maps (i.e. critical points for both inner and outer variations), and energy minimizing 1/2-harmonic maps. It can be seen as the analogue of the partial regularity theory for harmonic maps by R. Schoen and K. Uhlenbeck [48, 49] in the minimizing case, and by L.C. Evans [17] and F. Bethuel [1] in the stationary case. In [32], the argument consists in considering the harmonic extension to the upper half space in one more dimension provided by the convolution with the Poisson kernel. The extended map is then harmonic and satisfies a nonlinear Neumann boundary condition which fits within the (previously known) theory of harmonic maps with partially free boundary, see [15, 16, 21, 22, 43].

The purpose of this article is to extend the regularity theory for fractional harmonic maps in arbitrary dimensions to the context of $s$-harmonic maps, i.e., when the operator $(-\Delta)^{\frac{1}{2}}$ is replaced by $(-\Delta)^{s}$ with arbitrary power $s \in(0,1)$. As a first attempt in this direction, we only consider the case where the target manifold $\mathcal{N}$ is the standard unit sphere $\mathbb{S}^{d-1}$ of $\mathbb{R}^{d}, d \geqslant 2$. We now describe the functional setting.

Given $s \in(0,1)$ and $\Omega \subseteq \mathbb{R}^{n}$ a bounded open set, the fractional $s$-Dirichlet energy in $\Omega$ of a measurable map $u: \mathbb{R}^{n} \rightarrow \mathbb{R}^{d}$ is defined by

$$
\mathcal{E}_{s}(u, \Omega):=\frac{\gamma_{n, s}}{4} \iint_{\left(\mathbb{R}^{n} \times \mathbb{R}^{n}\right) \backslash\left(\Omega^{c} \times \Omega^{c}\right)} \frac{|u(x)-u(y)|^{2}}{|x-y|^{n+2 s}} \mathrm{~d} x \mathrm{~d} y,
$$

where $\Omega^{c}$ denotes the complement of $\Omega$, i.e. $\Omega^{c}:=\mathbb{R}^{n} \backslash \Omega$. The normalisation constant $\gamma_{n, s}>0$, whose precise value is given by $(2.1)$, is chosen in such a way that

$$
\mathcal{E}_{s}(u, \Omega)=\frac{1}{2} \int_{\mathbb{R}^{n}}\left|(-\Delta)^{\frac{s}{2}} u\right|^{2} \mathrm{~d} x \quad \forall u \in \mathscr{D}\left(\Omega ; \mathbb{R}^{d}\right) .
$$

Following $[32,33]$, we denote by $\widehat{H}^{s}\left(\Omega ; \mathbb{R}^{d}\right)$ the Hilbert space made of $L_{\text {loc }}^{2}\left(\mathbb{R}^{n}\right)$-maps $u$ such that $\mathcal{E}_{s}(u, \Omega)<\infty$, and we set

$$
\widehat{H}^{s}\left(\Omega ; \mathbb{S}^{d-1}\right):=\left\{u \in \widehat{H}^{s}\left(\Omega ; \mathbb{R}^{d}\right): u(x) \in \mathbb{S}^{d-1} \text { for a.e. } x \in \mathbb{R}^{n}\right\} .
$$

We then define weakly $s$-harmonic maps in $\Omega$ as critical points of $\mathcal{E}_{s}(u, \Omega)$ in the (nonlinear) space $\widehat{H}^{s}\left(\Omega ; \mathbb{S}^{d-1}\right)$. More precisely, we say that a map $u \in \widehat{H}^{s}\left(\Omega ; \mathbb{S}^{d-1}\right)$ is a weakly $s$-harmonic map in $\Omega$ into $\mathbb{S}^{d-1}$ if

$$
\left[\frac{\mathrm{d}}{\mathrm{d} t} \mathcal{E}_{s}\left(\frac{u+t \varphi}{|u+t \varphi|}, \Omega\right)\right]_{t=0}=0 \quad \forall \varphi \in \mathscr{D}\left(\Omega, \mathbb{R}^{d}\right) .
$$

Exactly as (1.2), the Euler-Lagrange equation reads

$$
(-\Delta)^{s} u(x)=\left(\frac{\gamma_{n, s}}{2} \int_{\mathbb{R}^{n}} \frac{|u(x)-u(y)|^{2}}{|x-y|^{n+2 s}} \mathrm{~d} y\right) u(x) \quad \text { in } \mathscr{D}^{\prime}(\Omega),
$$


where $(-\Delta)^{s}$ is the integro-differential operator given by

$$
(-\Delta)^{s} u(x):=\text { p.v. }\left(\gamma_{n, s} \int_{\mathbb{R}^{n}} \frac{u(x)-u(y)}{|x-y|^{n+2 s}} \mathrm{~d} y\right),
$$

and the notation p.v. means that the integral is taken in the Cauchy principal value sense. We refer to Section 2 and 3 for the precise weak (variational) formulation of equation (1.4).

Once again, the right hand side in (1.4) has a priori no better integrability than $L^{1}$, and linear elliptic theory does not apply to determine the regularity of solutions. However, in the case $n \leqslant 2 s$, that is $n=1$ and $s \in[1 / 2,1)$, the equation is subcritical. For $n=1$ and $s=1 / 2$, this is the result of $[9,10]$. For $n=1$ and $s \in(1 / 2,1)$, solutions are at least Hölder continuous by the embedding $H^{s} \hookrightarrow C^{0, s-1 / 2}$, and this is enough to reach $C^{\infty}$-smoothness by applying Schauder type estimates for the fractional Laplacian.

Theorem 1.1. Assume that $n=1$ and $s \in[1 / 2,1)$. If $u \in \widehat{H}^{s}\left(\Omega ; \mathbb{S}^{d-1}\right)$ is a weakly s-harmonic map in $\Omega$, then $u \in C^{\infty}(\Omega)$.

On the other hand, the case $n>2 s$ is supercritical, and by analogy with (usual) weakly harmonic maps in dimension at least 3, we do not expect any regularity without further assumptions. Indeed, in his groundbreaking article [38], T. Rivière has constructed a weakly harmonic map from the 3-dimensional ball into $\mathbb{S}^{2}$ which is everywhere discontinuous. A natural extra assumption to assume on a weakly $s$-harmonic map is stationarity, that is

$$
\left[\frac{\mathrm{d}}{\mathrm{d} t} \mathcal{E}_{s}\left(u \circ \phi_{t}, \Omega\right)\right]_{t=0}=0 \quad \forall X \in C_{c}^{1}\left(\Omega ; \mathbb{R}^{n}\right),
$$

where $\left\{\phi_{t}\right\}_{t \in \mathbb{R}}$ denotes the integral flow of the vector field $X$. According to the standard terminology in calculus of variations, a weakly $s$-harmonic map in $\Omega$ is a critical point of $\mathcal{E}_{s}(\cdot, \Omega)$ with respect to outer variations (i.e. in the target), a stationary map is a critical point of $\mathcal{E}_{s}(\cdot, \Omega)$ with respect to inner variations (i.e. in the domain), and thus a stationary weakly $s$-harmonic map in $\Omega$ is a critical point of $\mathcal{E}_{s}(\cdot, \Omega)$ with respect to both inner and outer variations.

Our second main result provides partial regularity for such maps. In its statement, the singular set of $u$ in $\Omega$ is defined as

$$
\operatorname{sing}(u):=\Omega \backslash\{x \in \Omega: u \text { is continuous in a neighborhood of } x\},
$$

$\operatorname{dim}_{\mathcal{H}}$ denotes the Hausdorff dimension, and $\mathcal{H}^{n-1}$ is the $(n-1)$-dimensional Hausdorff measure.

Theorem 1.2. Assume that $s \in(0,1)$ and $n>2 s$. If $u \in \widehat{H}^{s}\left(\Omega ; \mathbb{S}^{d-1}\right)$ is a stationary weakly $s$-harmonic map in $\Omega$, then $u \in C^{\infty}(\Omega \backslash \operatorname{sing}(u))$ and

(1) for $s>1 / 2$ and $n \geqslant 3, \operatorname{dim}_{\mathcal{H}} \operatorname{sing}(u) \leqslant n-2$;

(2) for $s>1 / 2$ and $n=2, \operatorname{sing}(u)$ is locally finite in $\Omega$;

(3) for $s=1 / 2$ and $n \geqslant 2, \mathcal{H}^{n-1}(\operatorname{sing}(u))=0$;

(4) for $s<1 / 2$ and $n \geqslant 2, \operatorname{dim}_{\mathcal{H}} \operatorname{sing}(u) \leqslant n-1$;

(5) for $s<1 / 2$ and $n=1, \operatorname{sing}(u)$ is locally finite in $\Omega$.

The other common assumption to consider is energy minimality. We say that a map $u \in$ $\widehat{H}^{s}\left(\Omega ; \mathbb{S}^{d-1}\right)$ is a minimizing $s$-harmonic map in $\Omega$ if

$$
\mathcal{E}_{s}(u, \Omega) \leqslant \mathcal{E}_{s}(v, \Omega)
$$

for every competitor $v \in \widehat{H}^{s}\left(\Omega ; \mathbb{S}^{d-1}\right)$ such that $v-u$ is compactly supported in $\Omega$. Notice that minimality implies criticality with respect to both inner and outer variations, so that a minimizing $s$-harmonic map in $\Omega$ is in particular a stationary weakly $s$-harmonic map in $\Omega$. However, minimality implies a stronger partial regularity, at least for $s \in(0,1 / 2)$.

Theorem 1.3. Assume that $s \in(0,1)$ and $n>2 s$. If $u \in \widehat{H}^{s}\left(\Omega ; \mathbb{S}^{d-1}\right)$ is a minimizing s-harmonic map in $\Omega$, then $u \in C^{\infty}(\Omega \backslash \operatorname{sing}(u))$ and

(1) for $n \geqslant 3, \operatorname{dim}_{\mathcal{H}} \operatorname{sing}(u) \leqslant n-2$;

(2) for $n=2, \operatorname{sing}(u)$ is locally finite in $\Omega$;

(3) for $n=1, \operatorname{sing}(u)=\emptyset$ (i.e., $u \in C^{\infty}(\Omega)$ ). 
Before describing the way we prove Theorem 1.2 and Theorem 1.3, let us comment on the sharpness of the results above.

Remark 1.4. In the case $s \in(0,1 / 2)$, essentially no better regularity than the one coming from the energy space can be expected from a weakly $s$-harmonic map in $\Omega$. Indeed, for an arbitrary set $E \subseteq \mathbb{R}^{n}$ such that the characteristic function $\chi_{E}$ belongs to $\widehat{H}^{s}(\Omega)$, consider the function $u:=\chi_{E}-\chi_{E^{c}}$. Identifying $\mathbb{R}^{2}$ with the complex plane $\mathbb{C}$, we can see $u$ as a map from $\mathbb{R}^{n}$ into $\mathbb{S}^{1}$, and it belongs to $\widehat{H}^{s}\left(\Omega ; \mathbb{S}^{1}\right)$. It has been observed in [33, Remark 1.7] that $u$ is a weakly $s$-harmonic map in $\Omega$ into $\mathbb{S}^{1}$, i.e., it satisfies (1.4). For $s=1 / 2$, we believe that, in the spirit of [38], it should be possible to construct an example of a 1/2-harmonic map from the 2-dimensional disc into $\mathbb{S}^{1}$ which is discontinuous everywhere using the material in [31]. However, for $s \in(1 / 2,1)$ and $n=2$, it remains open whether or not such pathological example do exist.

Remark 1.5. For $s \in(0,1 / 2)$, the partial regularity for stationary weakly $s$-harmonic maps is sharp in the sense that the size of the singular set can not be improved. Following Remark 1.4 above and [33, Remark 1.7], for a set $E \subseteq \mathbb{R}^{n}$ such that $\chi_{E} \in \widehat{H}^{s}(\Omega)$, the map $u:=\chi_{E}-\chi_{E^{c}}$ is a weakly $s$-harmonic map in $\Omega$ into $\mathbb{S}^{1}$, and

$$
\mathcal{E}_{s}(u, \Omega)=\gamma_{n, s} P_{2 s}(E, \Omega),
$$

where $P_{2 s}(E, \Omega)$ is the fractional $2 s$-perimeter of $E$ in $\Omega$ introduced by L. Caffarelli, J.M. Roquejoffre, and O. Savin in [2], and it is given by

$$
P_{2 s}(E, \Omega)=\left(\iint_{(E \cap \Omega) \times\left(E^{c} \cap \Omega\right)}+\iint_{\left(E \cap \Omega^{c}\right) \times\left(E^{c} \cap \Omega\right)}+\iint_{(E \cap \Omega) \times\left(E^{c} \cap \Omega^{c}\right)}\right) \frac{\mathrm{d} x \mathrm{~d} y}{|x-y|^{n+2 s}} .
$$

Therefore, $u$ is a stationary weakly $s$-harmonic map in $\Omega$ if and only if $E$ is stationary in $\Omega$ for the shape functional $P_{2 s}(\cdot, \Omega)$ (see [33]). This includes the case where $\partial E$ is a nonlocal minimal surface in the sense of [2]. In particular, if $E$ is a half space, then $u$ is a stationary weakly $s$-harmonic map in $\Omega$, and $\operatorname{sing}(u)=\partial E \cap \Omega$ is an hyperplane.

Remark 1.6. For arbitrary spheres, Theorem 1.3 is sharp for $s=1 / 2$, see Example 7.15. This example is built on the minimality of the map $x /|x|$ from the plane $\mathbb{R}^{2}$ into $\mathbb{S}^{1}$ proved in $[30$, Theorem 1.4]. The minimality of $x /|x|$ for $s \neq 1 / 2$ is open, but one can check that it is at least a stationary $s$-harmonic map into $\mathbb{S}^{1}$ in the unit disc $D_{1} \subseteq \mathbb{R}^{2}$, showing that Theorem 1.2 is sharp also for $s \in[1 / 2,1)$.

For arbitrary $s \in(0,1)$, the following classical example suggests that Theorem 1.3 might be sharp anyway. Consider the minimization problem (still in dimension $n=2$ ),

$$
\min \left\{\mathcal{E}_{s}\left(u, D_{1}\right): u \in \widehat{H}^{s}\left(D_{1}, \mathbb{S}^{1}\right), u(x)=x /|x| \text { in } \mathbb{R}^{2} \backslash D_{1}\right\} .
$$

Existence of solutions follows easily from the direct method of calculus of variations, and any solution is obviously a minimizing $s$-harmonic map in $D_{1}$. Since $x /|x|$ does not admit any $\mathbb{S}^{1}$-valued continuous extension to $D_{1}$, any solution must have at least one singular point in $\bar{D}_{1}$.

Remark 1.7. For $s=1 / 2$ and $d \geqslant 3$ (i.e., for $\mathbb{S}^{2}$ or higher dimensional target spheres), the size of the singular set of a minimizing $1 / 2$-harmonic map can be reduced. It has been proved in $[30$, Theorem 1.3] that in this case, $\operatorname{sing}(u)=\emptyset$ for $n=2$, it is locally finite for $n=3$, and $\operatorname{dim}_{\mathcal{H}} \operatorname{sing}(u) \leqslant n-3$ for $n \geqslant 4$. It would be interesting to know if this improvement persists for $s \neq 1 / 2$.

The proofs of Theorems 1.1, 1.2, and 1.3 rely on several ingredients that we now briefly describe. The first one consists in applying the so-called Caffarelli-Silvestre extension procedure [3] to the open half space $\mathbb{R}_{+}^{n+1}:=\mathbb{R}^{n} \times(0,+\infty)$. This extension (which may have originated in the probability literature [37]) allows us to represent $(-\Delta)^{s}$ as the Dirichlet-to-Neumann operator associated with the degenerate elliptic operator $L_{s}:=-\operatorname{div}\left(z^{1-2 s} \nabla \cdot\right)$, where $z \in(0,+\infty)$ denotes the extension variable. In this way (after extension), we can reformulate the $s$-harmonic map equation as a degenerate harmonic map equation with partially free boundary, very much like in $[32,33]$. Under the stationarity assumption, the extended map satisfies a fundamental monotonicity formula, which in turn implies local controls in the space BMO (bounded mean oscillation) of the $s$-harmonic map under consideration by its energy. 
Probably the main step in the proof is an epsilon-regularity result where we show that under a (standard) smallness assumption on the energy $\mathcal{E}_{s}$ in a ball, then a (stationary) $s$-harmonic map is Hölder continuous in a smaller ball. The strategy we follow here is quite inspired from the argument of L.C. Evans [17] making use of the conservation laws discovered by F. Hélein [24] and the duality $\mathcal{H}^{1} /$ BMO. In our fractional setting, we make use of the fractional conservation laws together with the "fractional div-curl lemma" of K. Mazowiecka and the third author [29]. A main difference with [17] lies in the fact that an additional "error term" appears when rewriting the $s$-harmonic map equation in the suitable form where compensation can be seen. To control this error term in arbitrary dimensions, we make use of a recent embedding result between Triebel-Lizorkin-Morrey type spaces [26] and various characterizations of these spaces [42, 57].

Once Hölder continuity is obtained, we prove Lipschitz continuity in an even smaller ball using an adjustment of the classical "harmonic replacement" technique, see [47]. More precisely, using the extension, we adapt an argument due to J. Roberts [40] in the case of degenerate harmonic maps with free boundary (i.e., with homogeneous - degenerate - Neumann boundary condition). With Lipschitz continuity in hands, we are then able to derive $C^{\infty}$-regularity from Schauder estimates for the fractional Laplacian.

To obtain the bounds on the size of the singular set, we follow somehow the usual dimension reduction argument of Almgren \& Federer for harmonic maps (see [53]), which is based on the strong compactness of blow-ups around points. Here compactness (for $s \neq 1 / 2$ ) is obtained as in [33], and it is a consequence of the monotonicity formula together with Marstrand's Theorem (see e.g. [28]). Finally, in the minimizing case and $s \in(0,1 / 2)$, we obtain an improvement on the size of the singular set (compared to the stationary case) from the triviality of the so-called "tangent maps" (i.e. blow-up limits), a consequence of the regularity of minimizing $s$-harmonic maps in one dimension proved in [34].

Notation. Throughout the paper, $\mathbb{R}^{n}$ is often identified with $\partial \mathbb{R}_{+}^{n+1}=\mathbb{R}^{n} \times\{0\}$. More generally, sets $A \subseteq \mathbb{R}^{n}$ can be identified with $A \times\{0\} \subseteq \partial \mathbb{R}_{+}^{n+1}$. Points in $\mathbb{R}^{n+1}$ are written $\mathbf{x}=(x, z)$ with $x \in \mathbb{R}^{n}$ and $z \in \mathbb{R}$. We shall denote by $B_{r}(\mathbf{x})$ the open ball in $\mathbb{R}^{n+1}$ of radius $r$ centered at $\mathbf{x}=(x, z)$, while $D_{r}(x):=B_{r}(\mathbf{x}) \cap \mathbb{R}^{n}$ is the open ball (or disc) in $\mathbb{R}^{n}$ centered at $x$. If $\mathbf{x}=0$, we simply write $B_{r}$ and $D_{r}$, respectively. For an arbitrary set $G \subseteq \mathbb{R}^{n+1}$, we write

$$
G^{+}:=G \cap \mathbb{R}_{+}^{n+1} \quad \text { and } \quad \partial^{+} G:=\partial G \cap \mathbb{R}_{+}^{n+1} .
$$

If $G \subseteq \mathbb{R}_{+}^{n+1}$ is a bounded open set, we shall say that $G$ is admissible whenever

- $\partial G$ is Lipschitz regular;

- the (relative) open set $\partial^{0} G \subseteq \partial \mathbb{R}_{+}^{n+1}$ defined by

$$
\partial^{0} G:=\left\{\mathbf{x} \in \partial G \cap \partial \mathbb{R}_{+}^{n+1}: B_{r}^{+}(\mathbf{x}) \subseteq G \text { for some } r>0\right\},
$$

is non empty and has Lipschitz boundary;

- $\partial G=\partial^{+} G \cup \overline{\partial^{0} G}$.

Finally, we usually denote by $C$ a generic positive constant which only depends on the dimension $n$ and $s \in(0,1)$, and possibly changing from line to line. If a constant depends on additional given parameters, we shall write those parameters using the subscript notation.

\section{Functional spaces, fractional operators, And COMPEnsated COMPACtness}

2.1. Fractional $H^{s}$-spaces. For an open set $\Omega \subseteq \mathbb{R}^{n}$, the Sobolev-Slobodeckij space $H^{s}(\Omega)$ is made of all functions $u \in L^{2}(\Omega)$ such that ${ }^{1}$

$$
[u]_{H^{s}(\Omega)}^{2}:=\frac{\gamma_{n, s}}{2} \iint_{\Omega \times \Omega} \frac{|u(x)-u(y)|^{2}}{|x-y|^{n+2 s}} \mathrm{~d} x \mathrm{~d} y<\infty, \quad \gamma_{n, s}:=s 2^{2 s} \pi^{-\frac{n}{2}} \frac{\Gamma\left(\frac{n+2 s}{2}\right)}{\Gamma(1-s)} .
$$

It is a separable Hilbert space normed by $\|\cdot\|_{H^{s}(\Omega)}^{2}:=\|\cdot\|_{L^{2}(\Omega)}^{2}+[\cdot]_{H^{s}(\Omega)}^{2}$. The space $H_{\mathrm{loc}}^{s}(\Omega)$ denotes the class of functions whose restriction to any relatively compact open subset $\Omega^{\prime}$ of $\Omega$ belongs to $H^{s}\left(\Omega^{\prime}\right)$. The linear subspace $H_{00}^{s}(\Omega) \subseteq H^{s}\left(\mathbb{R}^{n}\right)$ is in turn defined by

$$
H_{00}^{s}(\Omega):=\left\{u \in H^{s}\left(\mathbb{R}^{n}\right): u=0 \text { a.e. in } \mathbb{R}^{n} \backslash \Omega\right\} \text {. }
$$

\footnotetext{
${ }^{1}$ The normalization constant $\gamma_{n, s}$ is chosen in such a way that $[u]_{H^{s}\left(\mathbb{R}^{n}\right)}^{2}=\int_{\mathbb{R}^{n}}(2 \pi|\xi|)^{2 s}|\widehat{u}|^{2} \mathrm{~d} \xi$, where $\widehat{u}$ denotes the (ordinary frequency) Fourier transform of $u$.
} 
Endowed with the induced norm, $H_{00}^{s}(\Omega)$ is also a Hilbert space, and

$$
[u]_{H^{s}\left(\mathbb{R}^{n}\right)}^{2}=\frac{\gamma_{n, s}}{2} \iint_{\left(\mathbb{R}^{n} \times \mathbb{R}^{n}\right) \backslash\left(\Omega^{c} \times \Omega^{c}\right)} \frac{|u(x)-u(y)|^{2}}{|x-y|^{n+2 s}} \mathrm{~d} x \mathrm{~d} y=2 \mathcal{E}_{s}(u, \Omega) \quad \forall u \in H_{00}^{s}(\Omega),
$$

where $\mathcal{E}_{s}(\cdot, \Omega)$ is the $s$-Dirichlet energy defined in (1.3).

If $\Omega$ is bounded and its boundary is smooth enough (e.g. if $\partial \Omega$ is Lipschitz regular), then

$$
H_{00}^{s}(\Omega)=\overline{\mathscr{D}(\Omega)}\|\cdot\|_{H^{s}\left(\mathbb{R}^{n}\right)}
$$

(see [20, Theorem 1.4.2.2]) . The topological dual space of $H_{00}^{s}(\Omega)$ is denoted by $H^{-s}(\Omega)$.

We are mostly interested in the class of functions

$$
\widehat{H}^{s}(\Omega):=\left\{u \in L_{\mathrm{loc}}^{2}\left(\mathbb{R}^{n}\right): \mathcal{E}_{s}(u, \Omega)<\infty\right\} .
$$

The following properties hold for any open subsets $\Omega$ and $\Omega^{\prime}$ of $\mathbb{R}^{n}$ :

- $\widehat{H}^{s}(\Omega)$ is a linear space;

- $\widehat{H}^{s}(\Omega) \subseteq \widehat{H}^{s}\left(\Omega^{\prime}\right)$ whenever $\Omega^{\prime} \subseteq \Omega$, and $\mathcal{E}_{s}\left(\cdot, \Omega^{\prime}\right) \leqslant \mathcal{E}_{s}(\cdot, \Omega)$;

- if $\Omega^{\prime}$ is bounded, then $\widehat{H}^{s}(\Omega) \cap H_{\text {loc }}^{s}\left(\mathbb{R}^{n}\right) \subseteq \widehat{H}^{s}\left(\Omega^{\prime}\right)$;

- if $\Omega$ is bounded, then $H_{\mathrm{loc}}^{s}\left(\mathbb{R}^{n}\right) \cap L^{\infty}\left(\mathbb{R}^{n}\right) \subseteq \widehat{H}^{s}(\Omega)$,

where the third item follows from Lemma 2.1 below. Still by Lemma 2.1, $\widehat{H}^{s}(\Omega)$ is a Hilbert space for the scalar product induced by the norm $u \mapsto\|u\|_{\widehat{H}^{s}(\Omega)}:=\left(\|u\|_{L^{2}(\Omega)}^{2}+\mathcal{E}_{s}(u, \Omega)\right)^{1 / 2}$ (see e.g. [33] and [32, proof of Lemma 2.1]).

Lemma 2.1. Let $x_{0} \in \Omega$ and $\rho>0$ be such that $D_{\rho}\left(x_{0}\right) \subseteq \Omega$. There exists a constant $C_{\rho}=C_{\rho}(\rho, n, s)>0$ such that

$$
\int_{\mathbb{R}^{n}} \frac{|u(x)|^{2}}{\left(\left|x-x_{0}\right|+1\right)^{n+2 s}} \mathrm{~d} x \leqslant C_{\rho}\left(\mathcal{E}_{s}\left(u, D_{\rho}\left(x_{0}\right)\right)+\|u\|_{L^{2}\left(D_{\rho}\left(x_{0}\right)\right)}^{2}\right)
$$

for every $u \in \widehat{H}^{s}(\Omega)$.

Remark 2.2. Assume that $\Omega$ is bounded open set with a Lipschitz boundary. From the Hilbertian structure of $\widehat{H}^{s}(\Omega)$, it follows that any bounded sequence $\left\{u_{k}\right\}$ in $\widehat{H}^{s}(\Omega)$ admits a subsequence converging weakly in $\widehat{H}^{s}(\Omega)$. In addition, if $u_{k} \rightarrow u$ weakly in $\widehat{H}^{s}(\Omega)$, then $u_{k} \rightarrow u$ strongly in $L^{2}(\Omega)$ by the compact embedding $H^{s}(\Omega) \hookrightarrow L^{2}(\Omega)$ (see e.g. [20, Theorem 1.4.3.2]). In particular, $\left\|u_{k}\right\|_{L^{2}(\Omega)} \rightarrow\|u\|_{L^{2}(\Omega)}$. Since $\liminf _{k}\left\|u_{k}\right\|_{\hat{H}^{s}(\Omega)} \geqslant\|u\|_{\hat{H}^{s}(\Omega)}$, it follows that $\lim \inf _{k} \mathcal{E}_{s}\left(u_{k}, \Omega\right) \geqslant \mathcal{E}_{s}(u, \Omega)$.

2.2. Fractional operators and compensated compactness. Given an open set $\Omega \subseteq \mathbb{R}^{n}$, the fractional Laplacian $(-\Delta)^{s}$ in $\Omega$ is defined as the continuous linear operator $(-\Delta)^{s}$ : $\widehat{H}^{s}(\Omega) \rightarrow\left(\widehat{H}^{s}(\Omega)\right)^{\prime}$ induced by the quadratic form $\mathcal{E}_{s}(\cdot, \Omega)$. In other words, the weak form of the fractional Laplacian $(-\Delta)^{s} u$ of a given function $u \in \widehat{H}^{s}(\Omega)$ is defined through its action on $\widehat{H}^{s}(\Omega)$ by

$$
\left\langle(-\Delta)^{s} u, \varphi\right\rangle_{\Omega}:=\frac{\gamma_{n, s}}{2} \iint_{\left(\mathbb{R}^{n} \times \mathbb{R}^{n}\right) \backslash\left(\Omega^{c} \times \Omega^{c}\right)} \frac{(u(x)-u(y))(\varphi(x)-\varphi(y))}{|x-y|^{n+2 s}} \mathrm{~d} x \mathrm{~d} y .
$$

Notice that the restriction of the linear form $(-\Delta)^{s} u$ to the subspace $H_{00}^{s}(\Omega)$ belongs to $H^{-s}(\Omega)$ with the estimate $\left\|(-\Delta)^{s} u\right\|_{H^{-s}(\Omega)}^{2} \leqslant 2 \mathcal{E}_{s}(u, \Omega)$.

Remark 2.3. Notice the operator $(-\Delta)^{s}$ has the following local property: if $u \in \widehat{H}^{s}(\Omega)$ and $\Omega^{\prime} \subseteq \Omega$ is an open subset, then

$$
\left\langle(-\Delta)^{s} u, \varphi\right\rangle_{\Omega}=\left\langle(-\Delta)^{s} u, \varphi\right\rangle_{\Omega^{\prime}} \quad \forall \varphi \in H_{00}^{s}\left(\Omega^{\prime}\right) .
$$

Following [29], we now relate the fractional Laplacian $(-\Delta)^{s}$ to suitable notions of fractional gradient and fractional divergence. To this purpose, we first need to recall from [29] the notion of (fractional) "s-vector field" over a domain. The space of $s$-vector fields in $\Omega$, that we shall denote by $L_{\text {od }}^{2}(\Omega)$ (in agreement with [29]), is defined as the Lebesgue space of $L^{2}$-scalar functions over 
the open set $\left(\mathbb{R}^{n} \times \mathbb{R}^{n}\right) \backslash\left(\Omega^{c} \times \Omega^{c}\right) \subseteq \mathbb{R}^{2 n}$ with respect to the measure $|x-y|^{-n} \mathrm{~d} x \mathrm{~d} y$. In other words,

$$
L_{\text {od }}^{2}(\Omega):=\left\{F:\left(\mathbb{R}^{n} \times \mathbb{R}^{n}\right) \backslash\left(\Omega^{c} \times \Omega^{c}\right) \rightarrow \mathbb{R}:\|F\|_{L_{\text {od }}^{2}(\Omega)}<\infty\right\}
$$

with

$$
\|F\|_{L_{\mathrm{od}}^{2}(\Omega)}^{2}:=\iint_{\left(\mathbb{R}^{n} \times \mathbb{R}^{n}\right) \backslash\left(\Omega^{c} \times \Omega^{c}\right)} \frac{|F(x, y)|^{2}}{|x-y|^{n}} \mathrm{~d} x \mathrm{~d} y .
$$

We endow $L_{\text {od }}^{2}(\Omega)$ with the (pointwise) product operator $\odot: L_{\text {od }}^{2}(\Omega) \times L_{\text {od }}^{2}(\Omega) \times \rightarrow L^{1}(\Omega)$ given by

$$
F \odot G(x):=\int_{\mathbb{R}^{n}} \frac{F(x, y) G(x, y)}{|x-y|^{n}} \mathrm{~d} y .
$$

Note that $\odot$ is a continuous bilinear operator thanks to Fubini's theorem, and it plays the role of "pointwise scalar product" between two $s$-vector fields. With this respect, we define the (pointwise) "squared modulus" of a $s$-vector field $F \in L_{\text {od }}^{2}(\Omega)$ by

$$
|F|^{2}:=F \odot F \in L^{1}(\Omega) .
$$

The (fractional) $s$-gradient is defined in [29] as a linear operator from the space of scalar valued functions $\widehat{H}^{s}(\Omega)$ into the space of $s$-vector fields over $\Omega$. More precisely, we define it as the continuous linear operator $\mathrm{d}_{s}: \widehat{H}^{s}(\Omega) \rightarrow L_{\text {od }}^{2}(\Omega)$ given by

$$
\mathrm{d}_{s} u(x, y):=\frac{\sqrt{\gamma_{n, s}}}{\sqrt{2}} \frac{u(x)-u(y)}{|x-y|^{s}} .
$$

Obviously, one has

$$
\left\|\mathrm{d}_{s} u\right\|_{L_{\mathrm{od}}^{2}(\Omega)}^{2}=2 \mathcal{E}_{s}(u, \Omega) \quad \text { and } \quad\left\|\left|\mathrm{d}_{s} u\right|^{2}\right\|_{L^{1}(\Omega)} \leqslant 2 \mathcal{E}_{s}(u, \Omega)
$$

for every $u \in \widehat{H}^{s}(\Omega)$.

In turn, the (fractional) $s$-divergence, denoted by $\operatorname{div}_{s}$, is defined by duality as the adjoint operator to the $s$-gradient operator restricted to $H_{00}^{s}(\Omega)$. To do so, the main observation is that for $F \in L_{\text {od }}^{2}(\Omega)$, we have

$$
F \odot \mathrm{d}_{s} \varphi \in L^{1}\left(\mathbb{R}^{n}\right) \quad \text { for every } \varphi \in H_{00}^{s}(\Omega),
$$

with

$$
\left\|F \odot \mathrm{d}_{s} \varphi\right\|_{L^{1}\left(\mathbb{R}^{n}\right)} \leqslant\|F\|_{L_{\text {od }}^{2}(\Omega)}[\varphi]_{H^{s}\left(\mathbb{R}^{n}\right)} .
$$

In this way, we can indeed $\operatorname{define}_{\operatorname{div}_{s}}: L_{\text {od }}^{2}(\Omega) \rightarrow H^{-s}(\Omega)$ as the continuous linear operator given by

$$
\left\langle\operatorname{div}_{s} F, \varphi\right\rangle_{\Omega}:=\int_{\mathbb{R}^{n}} F \odot \mathrm{d}_{s} \varphi \mathrm{d} x \quad \forall \varphi \in H_{00}^{s}(\Omega),
$$

which satisfies the estimate $\left\|\operatorname{div}_{s} F\right\|_{H^{-s}(\Omega)} \leqslant\|F\|_{L_{\text {od }}^{2}(\Omega)}$ for all $F \in L_{\text {od }}^{2}(\Omega)$.

From the definition of $\mathrm{d}_{s}$ and $\operatorname{div}_{s}$, it readily follows that

Proposition 2.4. We have $(-\Delta)^{s}=\operatorname{div}_{s}\left(\mathrm{~d}_{s}\right)$, i.e.,

$$
\left\langle(-\Delta)^{s} u, \varphi\right\rangle_{\Omega}=\int_{\mathbb{R}^{n}} \mathrm{~d}_{s} u \odot \mathrm{d}_{s} \varphi \mathrm{d} x
$$

for every $u \in \widehat{H}^{s}(\Omega)$ and every $\varphi \in H_{00}^{s}(\Omega)$.

One of the main results in [29] is a compensated compactness result relative to the $s$-gradient and $s$-divergence operators in the spirit of the classical "div-curl" lemma [4]. To present this result, let us recall that the space $\operatorname{BMO}\left(\mathbb{R}^{n}\right)$ is defined as the set of all $u \in L_{\text {loc }}^{1}\left(\mathbb{R}^{n}\right)$ such that

$$
[u]_{\mathrm{BMO}\left(\mathbb{R}^{n}\right)}:=\sup _{D_{r}(y)} f_{D_{r}(y)}\left|u-(u)_{y, r}\right| \mathrm{d} x<+\infty,
$$

where $(u)_{y, r}$ denotes the average of $u$ over the ball $D_{r}(y)$. The following theorem corresponds to [29, Proposition 2.4]. 
Theorem 2.5. Let $F \in L_{\text {od }}^{2}(\Omega)$ be such that

$$
\operatorname{div}_{s} F=0 \quad \text { in } H^{-s}(\Omega) .
$$

There exist a universal $\Lambda>1$ such that for every ball $D_{r}\left(x_{0}\right)$ satisfying $D_{\Lambda r}\left(x_{0}\right) \subseteq \Omega$,

$$
\left|\int_{\mathbb{R}^{n}}\left(F \odot \mathrm{d}_{s} u\right) \varphi \mathrm{d} x\right| \leqslant C\|F\|_{L_{\text {od }}^{2}(\Omega)} \sqrt{\mathcal{E}_{s}(u, \Omega)}\left([\varphi]_{\mathrm{BMO}\left(\mathbb{R}^{\mathrm{n}}\right)}+r^{-n}\|\varphi\|_{L^{1}\left(\mathbb{R}^{n}\right)}\right)
$$

for every $u \in \widehat{H}^{s}(\Omega)$ and $\varphi \in \mathscr{D}\left(D_{r}\left(x_{0}\right)\right)$, and a constant $C=C(n, s)$.

Remark 2.6. In the statement of [29, Proposition 2.4], the $s$-vector field $F$ is assumed to be $s$-divergence free in the whole $\mathbb{R}^{n}$ and $u \in H^{s}\left(\mathbb{R}^{n}\right)$. However, a careful reading of the proof reveals that only the assumptions in Theorem 2.5 on $F$ and $u$ are used.

2.3. Weighted Sobolev spaces. For an open set $G \subseteq \mathbb{R}^{n+1}$, we consider the weighted $L^{2}$ space

normed by

$$
L^{2}\left(G,|z|^{a} \mathrm{~d} \mathbf{x}\right):=\left\{v \in L_{\mathrm{loc}}^{1}(G):|z|^{\frac{a}{2}} v \in L^{2}(G)\right\} \quad \text { with } a:=1-2 s,
$$

$$
\|v\|_{L^{2}\left(G,|z|^{a} \mathrm{~d} \mathbf{x}\right)}^{2}:=\int_{G}|z|^{a}|v|^{2} \mathrm{~d} \mathbf{x} .
$$

Accordingly, we introduce the weighted Sobolev space

$$
H^{1}\left(G,|z|^{a} \mathrm{~d} \mathbf{x}\right):=\left\{v \in L^{2}\left(G,|z|^{a} \mathrm{~d} \mathbf{x}\right): \nabla v \in L^{2}\left(G,|z|^{a} \mathrm{~d} \mathbf{x}\right)\right\},
$$

normed by

$$
\|v\|_{H^{1}\left(G,|z|^{a} \mathrm{~d} \mathbf{x}\right)}:=\|v\|_{L^{2}\left(G,|z|^{a} \mathrm{~d} \mathbf{x}\right)}+\|\nabla v\|_{L^{2}\left(G,|z|^{a} \mathrm{~d} \mathbf{x}\right)} .
$$

Both $L^{2}\left(G,|z|^{a} \mathrm{~d} \mathbf{x}\right)$ and $H^{1}\left(G,|z|^{a} \mathrm{~d} \mathbf{x}\right)$ are separable Hilbert spaces when equipped with the scalar product induced by their respective Hilbertian norms.

On $H^{1}\left(G,|z|^{a} \mathrm{~d} \mathbf{x}\right)$, we define the weighted Dirichlet energy $\mathbf{E}_{s}(\cdot, G)$ by setting

$$
\mathbf{E}_{s}(v, G):=\frac{\boldsymbol{\delta}_{s}}{2} \int_{G}|z|^{a}|\nabla v|^{2} \mathrm{~d} \mathbf{x} \quad \text { with } \boldsymbol{\delta}_{s}:=2^{2 s-1} \frac{\Gamma(s)}{\Gamma(1-s)} .
$$

The relevance of the normalisation constant $\boldsymbol{\delta}_{s}>0$ will be revealed in Section 2.4 (see (2.16)).

Some relevant remarks about $H^{1}\left(G,|z|^{a} \mathrm{~d} \mathbf{x}\right)$ are in order. For a bounded admissible open set $G \subseteq \mathbb{R}_{+}^{n+1}$, the space $L^{2}\left(G,|z|^{a} \mathrm{~d} \mathbf{x}\right)$ embeds continuously into $L^{\gamma}(G)$ for every $1 \leqslant \gamma<\frac{1}{1-s}$ whenever $s \in(0,1 / 2)$ by Hölder's inequality. For $s \in[1 / 2,1)$, we have $L^{2}\left(G,|z|^{a} \mathrm{~d} \mathbf{x}\right) \hookrightarrow L^{2}(G)$ continuously since $a \leqslant 0$. In any case, it implies that

$$
H^{1}\left(G,|z|^{a} \mathrm{~d} \mathbf{x}\right) \hookrightarrow W^{1, \gamma}(G)
$$

continuously for every $1<\gamma<\min \left\{\frac{1}{1-s}, 2\right\}$. As a first consequence, $H^{1}\left(G,|z|^{a} \mathrm{~d} \mathbf{x}\right) \hookrightarrow L^{1}(G)$ with compact embedding. Secondly, for such $\gamma$ 's, the compact linear trace operator

$$
v \in W^{1, \gamma}(G) \mapsto v_{\mid \partial^{\circ} G} \in L^{1}\left(\partial^{0} G\right)
$$

induces a compact linear trace operator from $H^{1}\left(G,|z|^{a} \mathrm{~d} \mathbf{x}\right)$ into $L^{1}\left(\partial^{0} G\right)$, extending the usual trace of smooth functions. We shall denote by $v_{\mid \partial^{0} G}$ the trace of $v \in H^{1}\left(G,|z|^{a} \mathrm{~d} \mathbf{x}\right)$ on $\partial^{0} G$, or simply by $v$ if it is clear from the context. We may now recall the following Poincaré's inequality, see e.g. [33, Lemma 2.5].

Lemma 2.7. If $v \in H^{1}\left(B_{r}^{+},|z|^{a} \mathrm{~d} \mathbf{x}\right)$, then

$$
\left\|v-(v)_{r}\right\|_{L^{1}\left(D_{r}\right)} \leqslant C r^{\frac{n+2 s}{2}}\|\nabla v\|_{L^{2}\left(B_{r}^{+},|z|^{a} \mathrm{~d} \mathbf{x}\right)},
$$

for a constant $C=C(n, s)$, where $(v)_{r}$ denotes the average of $v$ over $D_{r}$.

The next lemma states that the trace $v_{\mid \partial^{\circ} G}$ has actually $H^{s}$-regularity, at least locally.

Lemma 2.8. If $v \in H^{1}\left(B_{2 r}^{+},|z|^{a} \mathrm{~d} \mathbf{x}\right)$, then the trace of $v$ on $\partial^{0} B_{r}^{+} \simeq D_{r}$ belongs to $H^{s}\left(D_{r}\right)$, and

for a constant $C=C(n, s)$.

$$
[v]_{H^{s}\left(D_{r}\right)}^{2} \leqslant C \mathbf{E}_{s}\left(v, B_{2 r}^{+}\right),
$$


Proof. The proof follows exactly the one in [34, Lemma 2.3] which is stated only in dimension $n=1$. We reproduce the proof (in arbitrary dimension) for convenience of the reader, slightly anticipating a well-known identity presented in Section 2.4 (see (2.16)).

Rescaling variables, we can assume that $r=1$. Moreover, we may assume without loss of generality that $v$ has a vanishing average over the half ball $B_{2}^{+}$. Let $\zeta \in C^{\infty}\left(B_{2} ;[0,1]\right)$ be a cut-off function such that $\zeta(\mathbf{x})=1$ for $|\mathbf{x}| \leqslant 1, \zeta(\mathbf{x})=0$ for $|\mathbf{x}| \geqslant 3 / 2$. The function $v_{*}:=\zeta v$ belongs to $H^{1}\left(\mathbb{R}_{+}^{n+1},|z|^{a} \mathrm{~d} \mathbf{x}\right)$, and Poincaré's inequality in $H^{1}\left(\mathbb{R}_{+}^{n+1},|z|^{a} \mathrm{~d} \mathbf{x}\right)$ (see e.g. [18]) yields

$$
\int_{\mathbb{R}_{+}^{n+1}} z^{a}\left|\nabla v_{*}\right|^{2} \mathrm{~d} \mathbf{x} \leqslant 2 \mathbf{E}_{s}\left(v, B_{2}^{+}\right)+C \int_{B_{2}^{+}} z^{a}|v|^{2} \mathrm{~d} \mathbf{x} \leqslant C \mathbf{E}_{s}\left(v, B_{2}^{+}\right),
$$

for a constant $C=C(\zeta, n, s)$. On the other hand, it follows from (2.16) in Section 2.4 below that

$$
\iint_{D_{1} \times D_{1}} \frac{|v(x)-v(y)|^{2}}{|x-y|^{n+2 s}} \mathrm{~d} x \mathrm{~d} y \leqslant \iint_{\mathbb{R}^{n} \times \mathbb{R}^{n}} \frac{\left|v_{*}(x)-v_{*}(y)\right|^{2}}{|x-y|^{n+2 s}} \mathrm{~d} x \mathrm{~d} y \leqslant C \mathbf{E}_{s}\left(v_{*}, \mathbb{R}_{+}^{n+1}\right) .
$$

Gathering (2.9) and (2.10) leads to the announced estimate.

2.4. Fractional harmonic extension and the Dirichlet-to-Neumann operator. Let us consider the so-called fractional Poisson kernel $\mathbf{P}_{n, s}: \mathbb{R}_{+}^{n+1} \rightarrow[0, \infty)$ defined by

$$
\mathbf{P}_{n, s}(\mathbf{x}):=\sigma_{n, s} \frac{z^{2 s}}{|\mathbf{x}|^{n+2 s}} \quad \text { with } \sigma_{n, s}:=\pi^{-\frac{n}{2}} \frac{\Gamma\left(\frac{n+2 s}{2}\right)}{\Gamma(s)}
$$

where $\mathbf{x}:=(x, z) \in \mathbb{R}_{+}^{n+1}:=\mathbb{R}^{n} \times(0, \infty)$. The choice of the constant $\sigma_{n, s}$ is made in such a way that $\int_{\mathbb{R}^{n}} \mathbf{P}_{n, s}(x, z) \mathrm{d} x=1$ for every $z>0$ (see e.g. the computation in Remark 7.14). As shown in [3] (see also [37]), the function $\mathbf{P}_{n, s}$ solves

$$
\begin{cases}\operatorname{div}\left(z^{a} \nabla \mathbf{P}_{n, s}\right)=0 & \text { in } \mathbb{R}_{+}^{n+1}, \\ \mathbf{P}_{n, s}=\delta_{0} & \text { on } \partial \mathbb{R}_{+}^{n+1},\end{cases}
$$

where $\delta_{0}$ denotes the Dirac distribution at the origin.

From now on, for a measurable function $u$ defined over $\mathbb{R}^{n}$, we shall denote by $u^{\mathrm{e}}$ its extension to the half-space $\mathbb{R}_{+}^{n+1}$ given by the convolution (in the $x$-variable) of $u$ with $\mathbf{P}_{n, s}$, i.e.,

$$
u^{\mathrm{e}}(x, z):=\sigma_{n, s} \int_{\mathbb{R}^{n}} \frac{z^{2 s} u(y)}{\left(|x-y|^{2}+z^{2}\right)^{\frac{n+2 s}{2}}} \mathrm{~d} y .
$$

Notice that $u^{\mathrm{e}}$ is well defined if $u$ belongs to the Lebesgue space $L^{1}$ over $\mathbb{R}^{n}$ with respect to the probability measure

$$
\mathfrak{m}_{s}:=\sigma_{n, s}\left(1+|y|^{2}\right)^{-\frac{n+2 s}{2}} \mathrm{~d} y .
$$

In particular, $u^{\mathrm{e}}$ can be defined whenever $u \in \widehat{H}^{s}(\Omega)$ for some non-empty open set $\Omega \subseteq \mathbb{R}^{n}$ by Lemma 2.1. Moreover, if $u \in L^{\infty}\left(\mathbb{R}^{n}\right)$, then $u^{\mathrm{e}} \in L^{\infty}\left(\mathbb{R}_{+}^{n+1}\right)$ and

$$
\left\|u^{\mathrm{e}}\right\|_{L^{\infty}\left(\mathbb{R}_{+}^{n+1}\right)} \leqslant\|u\|_{L^{\infty}\left(\mathbb{R}^{n}\right)} .
$$

For a function $u \in L^{1}\left(\mathbb{R}^{n}, \mathfrak{m}_{s}\right)$, the extension $u^{\mathrm{e}}$ has a pointwise trace on $\partial \mathbb{R}_{+}^{n+1} \simeq \mathbb{R}^{n}$ which is equal to $u$ at every Lebesgue point. In addition, $u^{\mathrm{e}}$ solves the equation

$$
\left\{\begin{array}{ll}
\operatorname{div}\left(z^{a} \nabla u^{\mathrm{e}}\right)=0 & \text { in } \mathbb{R}_{+}^{n+1}, \\
u^{\mathrm{e}}=u & \text { on } \partial \mathbb{R}_{+}^{n+1}
\end{array} .\right.
$$

By analogy with the standard case $s=1 / 2$ (for which (2.15) reduces to the Laplace equation), the map $u^{\mathrm{e}}$ is referred to as the fractional harmonic extension of $u$.

It has been proved in [3] that $u^{\mathrm{e}}$ belongs to the weighted space $H^{1}\left(\mathbb{R}_{+}^{n+1},|z|^{a} \mathrm{~d} \mathbf{x}\right)$ whenever $u \in H^{s}\left(\mathbb{R}^{n}\right)$. Extending a well-known identity for $s=1 / 2$, the $H^{s}$-seminorm of $u$ coincides up to a multiplicative constant with the weighted $L^{2}$-norm of $\nabla u^{\mathrm{e}}$, and $u^{\mathrm{e}}$ turns out to minimize the weighted Dirichlet energy among all possible extensions. In other words,

$$
[u]_{H^{s}\left(\mathbb{R}^{n}\right)}^{2}=\mathbf{E}_{s}\left(u^{\mathrm{e}}, \mathbb{R}_{+}^{n+1}\right)=\inf \left\{\mathbf{E}_{s}\left(v, \mathbb{R}_{+}^{n+1}\right): v \in H^{1}\left(\mathbb{R}_{+}^{n+1},|z|^{a} \mathrm{~d} \mathbf{x}\right), v=u \text { on } \mathbb{R}^{n}\right\}
$$

for every $u \in H^{s}\left(\mathbb{R}^{n}\right)$ (thanks to the choice of the normalisation factor $\boldsymbol{\delta}_{s}$ in $(2.6)$ ). 
If $u \in \widehat{H}^{s}(\Omega)$ for some open set $\Omega \subseteq \mathbb{R}^{n}$, we have the following estimates on $u^{\mathrm{e}}$, somehow extending the first equality in (2.16) to the localized setting.

Lemma 2.9. Let $\Omega \subseteq \mathbb{R}^{n}$ be an open set. For every $u \in \widehat{H}^{s}(\Omega)$, the extension $u^{\mathrm{e}}$ given by $(2.12)$ belongs to $H^{1}\left(G,|z|^{a} \mathrm{~d} \mathbf{x}\right) \cap L_{\text {loc }}^{2}\left(\overline{\mathbb{R}_{+}^{n+1}},|z|^{a} \mathrm{~d} \mathbf{x}\right)$ for every bounded admissible open set $G \subseteq \mathbb{R}_{+}^{n+1}$ satisfying $\overline{\partial^{0} G} \subseteq \Omega$. In addition, for every point $\mathbf{x}_{0}=\left(x_{0}, 0\right) \in \Omega \times\{0\}$ and $r>0$ such that $D_{3 r}\left(x_{0}\right) \subseteq \Omega$,

$$
\left\|u^{\mathrm{e}}\right\|_{L^{2}\left(B_{r}^{+}\left(\mathbf{x}_{0}\right),|z|^{a} \mathrm{~d} \mathbf{x}\right)}^{2} \leqslant C\left(r^{2} \mathcal{E}_{s}\left(u, D_{2 r}\left(x_{0}\right)\right)+r^{2-2 s}\|u\|_{L^{2}\left(D_{2 r}\left(x_{0}\right)\right)}^{2}\right),
$$

and

$$
\mathbf{E}_{s}\left(u^{\mathrm{e}}, B_{r}^{+}\left(\mathbf{x}_{0}\right)\right) \leqslant C \mathcal{E}_{s}\left(u, D_{2 r}\left(x_{0}\right)\right),
$$

for a constant $C=C(n, s)$.

Proof. Translating and rescaling variables, we can assume that $x_{0}=0$ and $r=1$. Then (2.17) follows from [33, Lemma 2.10] (which is stated for $s \in(0,1 / 2)$, but the proof is in fact valid for any $s \in(0,1))$. Denote by $\bar{u}$ the average of $u$ over $D_{2}$. Noticing that $(u-\bar{u})^{\mathrm{e}}=u^{\mathrm{e}}-\bar{u}$, and applying [33, Lemma 2.10] to $u-\bar{u}$ yields

$$
\mathbf{E}_{s}\left(u^{\mathrm{e}}, B_{1}^{+}\right) \leqslant C\left(\mathcal{E}_{s}\left(u, D_{2}\right)+\|u-\bar{u}\|_{L^{2}\left(D_{2}\right)}^{2}\right) .
$$

On the other hand, by Poincaré's inequality in $H^{s}\left(D_{2}\right)$, we have

$$
\|u-\bar{u}\|_{L^{2}\left(D_{2}\right)}^{2} \leqslant C[u]_{H^{s}\left(D_{2}\right)}^{2} \leqslant C \mathcal{E}_{s}\left(u, D_{2}\right)
$$

and (2.18) follows.

Corollary 2.10. Let $\Omega \subseteq \mathbb{R}^{n}$ be an open set, and $G \subseteq \mathbb{R}_{+}^{n+1}$ a bounded admissible open set such that $\overline{\partial^{0} G} \subseteq \Omega$. The extension operator $u \mapsto u^{\mathrm{e}}$ defines a continuous linear operator from $\widehat{H}^{s}(\Omega)$ into $H^{1}\left(G,|z|^{a} \mathrm{~d} \mathbf{x}\right)$.

Proof. Set $\delta:=\operatorname{dist}\left(\partial^{0} G, \Omega^{c}\right)$, and

$$
\begin{gathered}
h_{1}:=\min \left\{\frac{\delta}{12}, \inf \left\{\operatorname{dist}\left(\mathbf{x}, \partial \mathbb{R}_{+}^{n+1}\right): \mathbf{x}=(x, z) \in G, \operatorname{dist}\left((x, 0), \partial^{0} G\right) \geqslant \delta / 2\right\}\right\}>0, \\
h_{2}:=\sup \left\{\operatorname{dist}\left(\mathbf{x}, \partial \mathbb{R}_{+}^{n+1}\right): \mathbf{x}=(x, z) \in G\right\}<+\infty .
\end{gathered}
$$

We also consider a large radius $R>0$ in such a way that $G \subseteq D_{R} \times \mathbb{R}$, and we define

$$
\omega:=\left\{x \in \mathbb{R}^{n}: \operatorname{dist}\left((x, 0), \partial^{0} G\right)<\delta / 2\right\},
$$

and

$$
G_{*}:=\left(\omega \times\left(0, h_{1}\right]\right) \cup\left(D_{R} \times\left(h_{1}, h_{2}\right)\right) .
$$

By construction, $G_{*}$ is a bounded admissible open set satisfying $\overline{\partial^{0} G_{*}} \subseteq \Omega$ and $G \subseteq G_{*}$. Therefore, it is enough to show that the extension operator is continuous from $\widehat{H}^{s}(\Omega)$ into $H^{1}\left(G_{*},|z|^{a} \mathrm{~d} \mathbf{x}\right)$. In other words, we can assume without loss of generality that $G=G_{*}$.

Covering $\omega \times\left(0, h_{1}\right]$ by finitely many half balls $B_{\delta / 6}^{+}\left(\mathbf{x}_{\mathbf{i}}\right)$ with $\mathbf{x}_{i} \in \omega \times\{0\}$, and applying Lemma 2.9 in those balls, we infer that $u^{\mathrm{e}} \in H^{1}\left(\omega \times\left(0, h_{1}\right),|z|^{a} \mathrm{~d} \mathbf{x}\right)$, and

$$
\left\|u^{\mathrm{e}}\right\|_{H^{1}\left(\omega \times\left(0, h_{1}\right),|z|^{a} \mathrm{~d} \mathbf{x}\right)}^{2} \leqslant C_{G}\left(\mathcal{E}_{s}(u, \Omega)+\|u\|_{L^{2}(\Omega)}^{2}\right),
$$

for a constant $C_{G}=C_{G}(G, n, s)$.

On the other hand, one may derive from formula (2.12) and Jensen's inequality that

$$
\left|u^{\mathrm{e}}(\mathbf{x})\right|^{2}+\left|\nabla u^{\mathrm{e}}(\mathbf{x})\right|^{2} \leqslant C_{G} \int_{\mathbb{R}^{n}} \frac{|u(y)|^{2}}{\left(|x-y|^{2}+h_{1}^{2}\right)^{\frac{n+2 s}{2}}} \mathrm{~d} y \quad \forall \mathbf{x}=(x, z) \in D_{R} \times\left(h_{1}, h_{2}\right) .
$$

It then follows from Lemma 2.1 that $u^{\mathrm{e}} \in H^{1}\left(D_{R} \times\left(h_{1}, h_{2}\right),|z|^{a} \mathrm{~d} \mathbf{x}\right)$ with

$$
\left\|u^{\mathrm{e}}\right\|_{H^{1}\left(D_{R} \times\left(h_{1}, h_{2}\right),|z|^{a} \mathrm{~d} \mathbf{x}\right)}^{2} \leqslant C_{G}\left(\mathcal{E}_{s}(u, \Omega)+\|u\|_{L^{2}(\Omega)}^{2}\right),
$$

which completes the proof.

Another useful fact about the extension by convolution with $\mathbf{P}_{n, s}$, is that it preserves some local Hölder continuity. It is very classical and follows from the explicit formula (and regularity) of $\mathbf{P}_{n, s}$. Details are left to the reader. 
Lemma 2.11. If $u \in L^{\infty}\left(\mathbb{R}^{n}\right) \cap C^{0, \beta}\left(D_{R}\right)$ for some $\beta \in(0, \min (1,2 s))$, then $u^{\mathrm{e}} \in C^{0, \beta}\left(B_{R / 4}^{+}\right)$, and

$$
R^{\beta}\left[u^{\mathrm{e}}\right]_{C^{0, \beta}\left(B_{R / 4}^{+}\right)} \leqslant C_{\beta}\left(R^{\beta}[u]_{C^{0, \beta}\left(D_{R}\right)}+\|u\|_{L^{\infty}\left(\mathbb{R}^{n}\right)}\right),
$$

for a constant $C_{\beta}=C_{\beta}(\beta, n, s)$.

Let us now assume that $\Omega \subseteq \mathbb{R}^{n}$ is a bounded open set with Lipschitz boundary. If $u \in \widehat{H}^{s}(\Omega)$, the divergence free vector field $z^{a} \nabla u^{\mathrm{e}}$ admits a distributional normal trace on $\Omega$, that we denote by $\Lambda^{(2 s)} u$. More precisely, we define $\boldsymbol{\Lambda}^{(2 s)} u$ through its action on a test function $\varphi \in \mathscr{D}(\Omega)$ by setting

$$
\left\langle\Lambda^{(2 s)} u, \varphi\right\rangle_{\Omega}:=\int_{\mathbb{R}_{+}^{n+1}} z^{a} \nabla u^{\mathrm{e}} \cdot \nabla \Phi \mathrm{d} \mathbf{x}
$$

where $\Phi$ is any smooth extension of $\varphi$ compactly supported in $\mathbb{R}_{+}^{n+1} \cup \Omega$. Note that the righthand side of (2.20) is well defined by Lemma 2.9. By the divergence theorem, it is routine to check that the integral in (2.20) does not depend on the choice of the extension $\Phi$. It can be thought of as a fractional Dirichlet-to-Neumann operator. Indeed, whenever $u$ is smooth, the distribution $\boldsymbol{\Lambda}^{(2 s)} u$ is the pointwise-defined function given by

$$
\boldsymbol{\Lambda}^{(2 s)} u(x)=-\lim _{z \downarrow 0} z^{a} \partial_{z} u^{\mathrm{e}}(x, z)=2 s \lim _{z \downarrow 0} \frac{u^{\mathrm{e}}(x, 0)-u^{\mathrm{e}}(x, z)}{z^{2 s}}
$$

at each point $x \in \Omega$.

In the case $\Omega=\mathbb{R}^{n}$, it has been proved in [3] that $\boldsymbol{\Lambda}^{(2 s)}$ coincides with $(-\Delta)^{s}$, up to the multiplicative factor $\boldsymbol{\delta}_{s}$. In the localized setting, this identity still holds, see e.g. [33, Lemma 2.12] and [32, Lemma 2.9].

Lemma 2.12. If $\Omega \subseteq \mathbb{R}^{n}$ is a bounded open set with Lipschitz boundary, then

$$
(-\Delta)^{s}=\boldsymbol{\delta}_{s} \boldsymbol{\Lambda}^{(2 s)} \text { on } \widehat{H}^{s}(\Omega) .
$$

One of the main consequences of Lemma 2.12 is a local counterpart of (2.16) concerning the minimality of $u^{\mathrm{e}}$. This is the purpose of Corollary 2.13 below, inspired from [2, Lemma 7.2], and taken from [33, Corollary 2.13].

Corollary 2.13. Let $\Omega \subseteq \mathbb{R}^{n}$ be a bounded open set, and $G \subseteq \mathbb{R}_{+}^{n+1}$ an admissible bounded open set such that $\overline{\partial^{0} G} \subseteq \Omega$. Let $u \in \widehat{H}^{s}\left(\Omega ; \mathbb{R}^{d}\right)$, and let $u^{\mathrm{e}}$ be its fractional harmonic extension to $\mathbb{R}_{+}^{n+1}$ given by $(2.12)$. Then,

$$
\mathbf{E}_{s}(v, G)-\mathbf{E}_{s}\left(u^{\mathrm{e}}, G\right) \geqslant \mathcal{E}_{s}(v, \Omega)-\mathcal{E}_{s}(u, \Omega)
$$

for all $v \in H^{1}\left(G ; \mathbb{R}^{d},|z|^{a} \mathrm{~d} \mathbf{x}\right)$ such that $v-u^{\mathrm{e}}$ is compactly supported in $G \cup \partial^{0} G$. In the right-hand side of (2.21), the trace of $v$ on $\partial^{0} G$ is extended by $u$ outside $\partial^{0} G$.

2.5. Inner variations, monotonicity formula, and density functions. In this section, our main goal is to present the monotonicity formula satisfied by critical points of $\mathcal{E}_{s}(\cdot, \Omega)$ under inner variations, i.e., by stationary points. We start recalling the notion of first inner variation, and then give an explicit formula to represent it.

Definition 2.14. Let $\Omega \subseteq \mathbb{R}^{n}$ be a bounded open set. Given a map $u \in \widehat{H}^{s}\left(\Omega ; \mathbb{R}^{d}\right)$ and a vector field $X \in C^{1}\left(\mathbb{R}^{n} ; \mathbb{R}^{n}\right)$ compactly supported in $\Omega$, the first (inner) variation of $\mathcal{E}_{s}(\cdot, \Omega)$ at $u$ and evaluated at $X$ is defined as

$$
\delta \mathcal{E}_{s}(u, \Omega)[X]:=\left[\frac{\mathrm{d}}{\mathrm{d} t} \mathcal{E}_{s}\left(u \circ \phi_{-t}, \Omega\right)\right]_{t=0},
$$

where $\left\{\phi_{t}\right\}_{t \in \mathbb{R}}$ denotes the integral flow on $\mathbb{R}^{n}$ generated by $X$, i.e., for every $x \in \mathbb{R}^{n}$, the map $t \mapsto \phi_{t}(x)$ is defined as the unique solution of the ordinary differential equation

$$
\left\{\begin{array}{l}
\frac{\mathrm{d}}{\mathrm{d} t} \phi_{t}(x)=X\left(\phi_{t}(x)\right), \\
\phi_{0}(x)=x .
\end{array}\right.
$$

The following representation result for $\delta \mathcal{E}_{s}$ was obtained in [33, Corollary 2.14] as a direct consequence of Corollary 2.13. We reproduce here the proof for completeness. 
Proposition 2.15. Let $\Omega \subseteq \mathbb{R}^{n}$ be a bounded open set, and $G \subseteq \mathbb{R}_{+}^{n+1}$ an admissible bounded open set such that $\overline{\partial^{0} G} \subseteq \Omega$. For each $u \in \widehat{H}^{s}\left(\Omega ; \mathbb{R}^{d}\right)$, and each $X \in C^{1}\left(\mathbb{R}^{n} ; \mathbb{R}^{n}\right)$ compactly supported in $\partial^{0} G$, we have

$$
\begin{aligned}
\delta \mathcal{E}_{s}(u, \Omega)[X]=\frac{\boldsymbol{\delta}_{s}}{2} \int_{G} z^{a}\left(\left|\nabla u^{\mathrm{e}}\right|^{2} \operatorname{div} \mathbf{X}-2 \sum_{i, j=1}^{n+1}\left(\partial_{i} u^{\mathrm{e}} \cdot \partial_{j} u^{\mathrm{e}}\right) \partial_{j} \mathbf{X}_{i}\right) \mathrm{d} \mathbf{x} \\
+\frac{\boldsymbol{\delta}_{s} a}{2} \int_{G} z^{a-1}\left|\nabla u^{\mathrm{e}}\right|^{2} \mathbf{X}_{n+1} \mathrm{~d} \mathbf{x},
\end{aligned}
$$

where $\mathbf{X}=\left(\mathbf{X}_{1}, \ldots, \mathbf{X}_{n+1}\right) \in C^{1}\left(\bar{G} ; \mathbb{R}^{n+1}\right)$ is any vector field compactly supported in $G \cup \partial^{0} G$, and satisfying $\mathbf{X}=(X, 0)$ on $\partial^{0} G$.

Proof. Let $\mathbf{X} \in C^{1}\left(\bar{G}, \mathbb{R}^{n+1}\right)$ be an arbitrary vector field compactly supported in $G \cup \partial^{0} G$ and satisfying $\mathbf{X}=(X, 0)$ on $\partial^{0} G$. We consider a compactly supported $C^{1}$-extension of $\mathbf{X}$ to the whole space $\mathbb{R}^{n+1}$, still denoted by $\mathbf{X}$, such that $\mathbf{X}=(X, 0)$ on $\mathbb{R}^{n} \times\{0\} \simeq \mathbb{R}^{n}$. We define $\left\{\boldsymbol{\Phi}_{t}\right\}_{t \in \mathbb{R}}$ as the integral flow on $\mathbb{R}^{n+1}$ generated by $\mathbf{X}$. Observe that $\boldsymbol{\Phi}_{t}=\left(\phi_{t}, 0\right)$ on $\mathbb{R}^{n}$, and $\operatorname{spt}\left(\boldsymbol{\Phi}_{t}-\operatorname{id}_{\mathbb{R}^{n+1}}\right) \cap \overline{\mathbb{R}_{+}^{n+1}} \subseteq G \cup \partial^{0} G$. Then, $v_{t}:=u^{\mathrm{e}} \circ \boldsymbol{\Phi}_{-t} \in H^{1}\left(G ; \mathbb{R}^{d},|z|^{a} \mathrm{~d} \mathbf{x}\right)$ and $\operatorname{spt}\left(v_{t}-u^{\mathrm{e}}\right) \subseteq G \cup \partial^{0} G$. By Corollary 2.13, we have

$$
\mathbf{E}_{s}\left(v_{t}, G\right)-\mathbf{E}_{s}\left(u^{\mathrm{e}}, G\right) \geqslant \mathcal{E}_{s}\left(v_{t}, \Omega\right)-\mathcal{E}_{s}(u, \Omega) \quad \forall t \in \mathbb{R} .
$$

Since $v_{t}=u \circ \phi_{-t}$ on $\mathbb{R}^{n}$, dividing both sides of (2.23) by $t \neq 0$, and letting $t \uparrow 0$ and $t \downarrow 0$ leads to

$$
\delta \mathcal{E}_{s}(u, \Omega)[X]=\left[\frac{\mathrm{d}}{\mathrm{d} t} \mathbf{E}_{s}\left(u^{\mathrm{e}} \circ \mathbf{\Phi}_{-t}, G\right)\right]_{t=0} .
$$

On the other hand, standard computations (see e.g. [53, Chapter 2.2]) show that the right-hand side of (2.24) is equal to the right-hand side of $(2.22)$.

Definition 2.16. Let $\Omega \subseteq \mathbb{R}^{n}$ be a bounded open set. A map $u \in \widehat{H}^{s}\left(\Omega ; \mathbb{R}^{d}\right)$ is said to be stationary in $\Omega$ if $\delta \mathcal{E}_{s}(u, \Omega)=0$.

As we shall see in the next sections, stationarity is a crucial ingredient in the partial regularity theory since it implies the aforementioned monotonicity formula. This is the purpose of the following proposition whose proof follows exactly [33, Proof of Lemma 4.2] using vector fields in $(2.22)$ of the form $\mathbf{X}=\eta\left(\left|\mathbf{x}-\mathbf{x}_{0}\right|\right)\left(\mathbf{x}-\mathbf{x}_{0}\right)$ with $\eta(t) \sim \chi_{[0, r]}(t)$.

Proposition 2.17. Let $\Omega \subseteq \mathbb{R}^{n}$ be a bounded open set. If $u \in \widehat{H}^{s}\left(\Omega ; \mathbb{R}^{d}\right)$ is stationary in $\Omega$, then for every $\mathbf{x}_{0}=\left(x_{0}, 0\right) \in \Omega \times\{0\}$, the "density function"

$$
r \in\left(0, \operatorname{dist}\left(x_{0}, \Omega^{c}\right)\right) \mapsto \boldsymbol{\Theta}_{s}\left(u^{\mathrm{e}}, \mathbf{x}_{0}, r\right):=\frac{1}{r^{n-2 s}} \mathbf{E}_{s}\left(u^{\mathrm{e}}, B_{r}^{+}\left(\mathbf{x}_{0}\right)\right)
$$

is nondecreasing. Moreover,

$$
\boldsymbol{\Theta}_{s}\left(u^{\mathrm{e}}, \mathbf{x}_{0}, r\right)-\boldsymbol{\Theta}_{s}\left(u^{\mathrm{e}}, \mathbf{x}_{0}, \rho\right)=\boldsymbol{\delta}_{s} \int_{B_{r}^{+}\left(\mathbf{x}_{0}\right) \backslash B_{\rho}^{+}\left(\mathbf{x}_{0}\right)} z^{a} \frac{\left|\left(\mathbf{x}-\mathbf{x}_{0}\right) \cdot \nabla u^{\mathrm{e}}\right|^{2}}{\left|\mathbf{x}-\mathbf{x}_{0}\right|^{n+2-2 s}} \mathrm{~d} \mathbf{x}
$$

for every $0<\rho<r<\operatorname{dist}\left(x_{0}, \Omega^{c}\right)$.

As a straightforward consequence, we have

Corollary 2.18. Let $\Omega \subseteq \mathbb{R}^{n}$ be a bounded open set. If $u \in \widehat{H}^{s}\left(\Omega ; \mathbb{R}^{d}\right)$ is stationary in $\Omega$, then for every $x_{0} \in \Omega$, the limit

$$
\boldsymbol{\Xi}_{s}\left(u, x_{0}\right):=\lim _{r \rightarrow 0} \boldsymbol{\Theta}_{s}\left(u^{\mathrm{e}},\left(x_{0}, 0\right), r\right)
$$

exists, and the function $\boldsymbol{\Xi}_{s}(u, \cdot): \Omega \rightarrow[0, \infty)$ is upper semicontinuous. In addition, for every $\mathbf{x}_{0}=\left(x_{0}, 0\right) \in \Omega \times\{0\}$

$$
\boldsymbol{\Theta}_{s}\left(u^{\mathrm{e}}, \mathbf{x}_{0}, r\right)-\boldsymbol{\Xi}_{s}\left(u, x_{0}\right)=\boldsymbol{\delta}_{s} \int_{B_{r}^{+}\left(\mathbf{x}_{0}\right)} z^{a} \frac{\left|\left(\mathbf{x}-\mathbf{x}_{0}\right) \cdot \nabla u^{\mathrm{e}}\right|^{2}}{\left|\mathbf{x}-\mathbf{x}_{0}\right|^{n+2-2 s}} \mathrm{~d} \mathbf{x}
$$

for every $0<r<\operatorname{dist}\left(x_{0}, \Omega^{c}\right)$. 
Proof. The existence of the limit in (2.25) and (2.26) are direct consequences of the monotonicity formula established in Proposition 2.17. Then the function $\boldsymbol{\Xi}_{s}(u, \cdot)$ is upper semicontinuous as a pointwise limit of a decreasing family of continuous functions.

As we previously said, the monotonicity of the density function $r \mapsto \boldsymbol{\Theta}_{s}\left(u^{\mathrm{e}}, \mathbf{x}_{0}, r\right)$ is one of the most important ingredients to obtain partial regularity. We shall see in the next sections that the density function relative to the nonlocal energy $\mathcal{E}_{s}$ also plays a role. For $u \in \widehat{H}^{s}\left(\Omega ; \mathbb{R}^{d}\right)$ and a point $x \in \Omega$, we define the density function $r \in\left(0, \operatorname{dist}\left(x, \Omega^{c}\right)\right) \mapsto \boldsymbol{\theta}_{s}(u, x, r)$ by setting

$$
\boldsymbol{\theta}_{s}\left(u, x_{0}, r\right):=\frac{1}{r^{n-2 s}} \mathcal{E}_{s}\left(u, D_{r}\left(x_{0}\right)\right) .
$$

Now we aim to show that one density function is small if and only the other one is also small at a comparable scale. This is the purpose of the following lemma.

Lemma 2.19. Let $\Omega \subseteq \mathbb{R}^{n}$ be an open set, and $u \in \widehat{H}^{s}\left(\Omega ; \mathbb{R}^{d}\right) \cap L^{\infty}\left(\mathbb{R}^{n}\right)$ be such that $\|u\|_{L^{\infty}\left(\mathbb{R}^{n}\right)} \leqslant M$. For every $\varepsilon>0$, there exists $\delta=\delta(n, s, M, \varepsilon)>0$ and $\alpha=\alpha(n, s, M, \varepsilon) \in$ $(0,1 / 4]$ such that

$$
\boldsymbol{\Theta}_{s}\left(u^{\mathrm{e}}, \mathbf{x}_{0}, r\right) \leqslant \delta \quad \Longrightarrow \quad \boldsymbol{\theta}_{s}\left(u, x_{0}, \alpha r\right) \leqslant \varepsilon
$$

for every $\mathbf{x}_{0}=\left(x_{0}, 0\right) \in \Omega \times\{0\}$ and $r>0$ satisfying $\bar{D}_{r}\left(x_{0}\right) \subseteq \Omega$.

Proof. Without loss of generality, we can assume that $x_{0}=0$. We give ourselves $\varepsilon>0$, and we shall choose the parameter $\alpha \in(0,1 / 4]$ later on. Using Lemma 2.8, we first estimate

$$
\begin{aligned}
\mathcal{E}_{s}\left(u, D_{\alpha r}\right) & \leqslant \frac{\gamma_{n, s}}{4} \iint_{D_{r / 2} \times D_{r / 2}} \frac{|u(x)-u(y)|^{2}}{|x-y|^{n+2 s}} \mathrm{~d} x \mathrm{~d} y+\frac{\gamma_{n, s}}{2} \iint_{D_{\alpha r} \times D_{r / 2}^{c}} \frac{|u(x)-u(y)|^{2}}{|x-y|^{n+2 s}} \mathrm{~d} x \mathrm{~d} y \\
& \leqslant C_{1} \mathbf{E}_{s}\left(u^{\mathrm{e}}, B_{r}^{+}\right)+2 M^{2} \gamma_{n, s} \iint_{D_{\alpha r} \times D_{r / 2}^{c}} \frac{\mathrm{d} x \mathrm{~d} y}{|x-y|^{n+2 s}},
\end{aligned}
$$

where $C_{1}=C_{1}(n, s)>0$. Observe that for $(x, y) \in D_{\alpha r} \times D_{r / 2}^{c}$, we have $|x-y| \geqslant|y|-\alpha r \geqslant \frac{1}{2}|y|$, so that

$$
2 \gamma_{n, s} \iint_{D_{\alpha r} \times D_{r / 2}^{c}} \frac{\mathrm{d} x \mathrm{~d} y}{|x-y|^{n+2 s}} \leqslant 2^{n+2 s+1} \gamma_{n, s} \iint_{D_{\alpha r} \times D_{r / 2}^{c}} \frac{\mathrm{d} x \mathrm{~d} y}{|y|^{n+2 s}}=C_{2} \alpha^{n} r^{n-2 s},
$$

where $C_{2}=C_{2}(n, s)>0$. Consequently,

$$
\boldsymbol{\theta}_{s}(u, 0, \alpha r) \leqslant \frac{C_{1}}{\alpha^{n-2 s}} \boldsymbol{\Theta}_{s}\left(u^{\mathrm{e}}, 0, r\right)+C_{2} M^{2} \alpha^{2 s} .
$$

Choosing

$$
\alpha=\min \left\{1 / 4,\left(\frac{\varepsilon}{2 C_{2} M^{2}}\right)^{1 / 2 s}\right\} \quad \text { and } \quad \delta:=\frac{\alpha^{n-2 s} \varepsilon}{2 C_{1}},
$$

provides the desired conclusion.

Corollary 2.20. Let $\Omega \subseteq \mathbb{R}^{n}$ be an open set. If $u \in \widehat{H}^{s}\left(\Omega ; \mathbb{R}^{d}\right) \cap L^{\infty}\left(\mathbb{R}^{n}\right)$, then

$$
\lim _{r \rightarrow 0} \boldsymbol{\theta}_{s}\left(u, x_{0}, r\right)=0 \Longleftrightarrow \lim _{r \rightarrow 0} \Theta_{s}\left(u^{\mathrm{e}}, \mathbf{x}_{0}, r\right)=0
$$

for every $\mathbf{x}_{0}=\left(x_{0}, 0\right) \in \Omega \times\{0\}$.

Proof. By Lemma 2.9, we have

$$
\boldsymbol{\Theta}_{s}\left(u^{\mathrm{e}}, \mathbf{x}_{0}, r\right) \leqslant C \boldsymbol{\theta}_{s}\left(u, x_{0}, 2 r\right),
$$

for a constant $C>0$ depending only on $n$ and $s$, and implication $\Longrightarrow$ follows. The reverse implication is a straightforward application of Lemma 2.19 . 
2.6. Energy monotonicity and mean oscillation estimates. In the light of Proposition 2.17, the purpose of this section is to show a mean oscillation estimate for maps having a nondecreasing density function at every point. For $v \in H^{1}\left(B_{R}^{+} ; \mathbb{R}^{d},|z|^{a} \mathrm{~d} \mathbf{x}\right)$, a point $\mathbf{x}_{0} \in \partial^{0} B_{R}^{+}$, and $r \in\left(0, R-\left|\mathbf{x}_{0}\right|\right)$, we keep the notation

$$
\mathbf{\Theta}_{s}\left(v, \mathbf{x}_{0}, r\right):=\frac{1}{r^{n-2 s}} \mathbf{E}_{s}\left(v, B_{r}^{+}\left(\mathbf{x}_{0}\right)\right) .
$$

The main estimate is the following.

Lemma 2.21. Let $v \in H^{1}\left(B_{R}^{+} ; \mathbb{R}^{d},|z|^{a} \mathrm{~d} \mathbf{x}\right)$ and $\zeta \in \mathscr{D}\left(D_{5 R / 8}\right)$ be such that $0 \leqslant \zeta \leqslant 1, \zeta \equiv 1$ in $D_{R / 2}$, and $|\nabla \zeta| \leqslant L R^{-1}$ for some constant $L>0$. Assume that for every $\mathbf{x} \in \partial^{0} B_{R}^{+}$, the density function $r \in(0, R-|\mathbf{x}|) \mapsto \boldsymbol{\Theta}_{s}(v, \mathbf{x}, r)$ is non decreasing. Then $(\zeta v)_{\mathbb{R}^{n}}$ belongs to $\operatorname{BMO}\left(\mathbb{R}^{n}\right)$ and

$$
[\zeta v]_{\mathrm{BMO}\left(\mathbb{R}^{n}\right)}^{2} \leqslant C_{L}\left(\boldsymbol{\Theta}_{s}(v, 0, R)+R^{2 s-2-n}\|v\|_{L^{2}\left(B_{R}^{+},|z|^{a} \mathrm{~d} \mathbf{x}\right)}^{2}\right)
$$

for a constant $C_{L}=C(L, n, s)$.

Before proving this lemma, let us recall that $u \in L^{1}\left(D_{R}\right)$ belongs to $\operatorname{BMO}\left(D_{R}\right)$ if

$$
[u]_{\mathrm{BMO}\left(D_{R}\right)}:=\sup _{D_{r}(y) \subseteq D_{R}} f_{D_{r}(y)}\left|u-(u)_{y, r}\right| \mathrm{d} x<+\infty,
$$

where $(u)_{y, r}$ denotes the average of $u$ over the ball $D_{r}(y)$. To prove Lemma 2.21, we shall make use of the well-known John-Nirenberg inequality, see e.g. [19, Section 6.3].

Lemma 2.22. Let $u \in \operatorname{BMO}\left(D_{R}\right)$. For every $p \in[1, \infty)$, there exists a constant $C_{p}=C_{p}(n, p)$ such that

$$
[u]_{\mathrm{BMO}\left(D_{R}\right)}^{p} \leqslant \sup _{D_{r}(y) \subseteq D_{R}} f_{D_{r}(y)}\left|u-(u)_{y, r}\right|^{p} \mathrm{~d} x \leqslant C_{p}[u]_{\mathrm{BMO}\left(D_{R}\right)}^{p} .
$$

Proof of Lemma 2.21. Step 1. Rescaling variables, we may assume that $R=1$. Let us fix an arbitrary ball $D_{r}(y) \subseteq D_{1}$ with $y \in D_{7 / 8}$ and $0<r \leqslant 1 / 8$. Using the Poincaré inequality in Lemma 2.7 and the monotonicity assumption on $\boldsymbol{\Theta}_{s}(v, \mathbf{x}, \cdot)$, we estimate

$$
\frac{1}{r^{n}} \int_{D_{r}(y)}\left|v-(v)_{y, r}\right| \mathrm{d} x \leqslant C \sqrt{\boldsymbol{\Theta}_{s}(v, \mathbf{y}, r)} \leqslant C \sqrt{\boldsymbol{\Theta}_{s}(v, \mathbf{y}, 1 / 8)} \leqslant C \sqrt{\boldsymbol{\Theta}_{s}(v, 0,1)},
$$

where $\mathbf{y}=(y, 0)$ and $C=C(n, s)$. In particular, $v_{\mid D_{7 / 8}}$ belongs to $\operatorname{BMO}\left(D_{7 / 8}\right)$, and

$$
[v]_{\mathrm{BMO}\left(D_{7 / 8}\right)} \leqslant C \sqrt{\boldsymbol{\Theta}_{s}(v, 0,1)} .
$$

By the John-Nirenberg inequality in Lemma 2.22, inequality (2.28), the continuity of the trace operator (see Section 2.3), and Hölder's inequality, it follows that

$$
\begin{aligned}
\|v\|_{L^{n}\left(D_{7 / 8}\right)} & \leqslant\left\|v-(v)_{0,7 / 8}\right\|_{L^{n}\left(D_{7 / 8}\right)}+C\|v\|_{L^{1}\left(D_{7 / 8}\right)} \\
& \leqslant C\left([v]_{\operatorname{BMO}\left(D_{7 / 8}\right)}+\|v\|_{L^{1}\left(D_{1}\right)}\right) \leqslant C\left(\sqrt{\boldsymbol{\Theta}_{s}(v, 0,1)}+\|v\|_{L^{2}\left(B_{1}^{+},|z|^{a} \mathrm{~d} \mathbf{x}\right)}\right) .
\end{aligned}
$$

Step 2. Let us now consider a ball $D_{r}(y) \subseteq D_{7 / 8}$ with $y \in D_{3 / 4}$ and $0<r \leqslant 1 / 8$. Since

$$
\left|\zeta v-(\zeta v)_{y, r}\right| \leqslant\left|\zeta v-\zeta(v)_{y, r}\right|+\left|\zeta(v)_{y, r}-(\zeta v)_{y, r}\right| \leqslant\left|v-(v)_{y, r}\right|+L r f_{D_{r}(y)}|v| \mathrm{d} x \quad \text { on } D_{7 / 8},
$$

we can deduce from $(2.28)$ and $(2.29)$ that

$$
\begin{aligned}
\frac{1}{r^{n}} \int_{D_{r}(y)} \mid \zeta v & -(\zeta v)_{y, r} \mid \mathrm{d} x \leqslant C_{L}\left(\sqrt{\boldsymbol{\Theta}_{s}(v, 0,1)}+r^{1-n}\|v\|_{L^{1}\left(D_{r}(y)\right)}\right) \\
\leqslant & C_{L}\left(\sqrt{\boldsymbol{\Theta}_{s}(v, 0,1)}+\|v\|_{L^{n}\left(D_{7 / 8}\right)}\right) \leqslant C_{L}\left(\sqrt{\boldsymbol{\Theta}_{s}(v, 0,1)}+\|v\|_{L^{2}\left(B_{1}^{+},|z|^{a} \mathrm{~d} \mathbf{x}\right)}\right),
\end{aligned}
$$

for a constant $C_{L}=C(L, n, s)$.

Next, for a ball $D_{r}(y)$ with $y \notin D_{3 / 4}$ and $0<r \leqslant 1 / 8$, we have

$$
\frac{1}{r^{n}} \int_{D_{r}(y)}\left|\zeta v-(\zeta v)_{y, r}\right| \mathrm{d} x=0,
$$

since $\zeta$ is supported in $D_{5 / 8}$. 
Finally, for a ball $D_{r}(y)$ with $r>1 / 8$, we estimate

$$
\frac{1}{r^{n}} \int_{D_{r}(y)}\left|\zeta v-(\zeta v)_{y, r}\right| \mathrm{d} x \leqslant C \int_{D_{1}}|\zeta v| \mathrm{d} x \leqslant C\|v\|_{L^{1}\left(D_{1}\right)} \leqslant C\|v\|_{L^{2}\left(B_{1}^{+},|z|^{a} \mathrm{~d} \mathbf{x}\right)},
$$

which completes the proof.

Corollary 2.23. Let $u \in \widehat{H}^{s}\left(D_{2 R} ; \mathbb{R}^{d}\right)$ and $\zeta \in \mathscr{D}\left(D_{5 R / 8}\right)$ be as in Lemma 2.21. Assume that for every $\mathbf{x} \in \partial^{0} B_{R}^{+}$, the density function $r \in(0,2 R-|\mathbf{x}|) \mapsto \boldsymbol{\Theta}_{s}\left(u^{\mathrm{e}}, \mathbf{x}, r\right)$ is non decreasing. Then $\zeta u$ belongs to $\mathrm{BMO}\left(\mathbb{R}^{n}\right)$ and

$$
[\zeta u]_{\mathrm{BMO}\left(\mathbb{R}^{n}\right)}^{2} \leqslant C_{L}\left(\boldsymbol{\theta}_{s}(u, 0,2 R)+R^{-n}\|u\|_{L^{2}\left(D_{2 R}\right)}^{2}\right),
$$

for a constant $C_{L}=C(L, n, s)>0$.

Proof. Apply Lemma 2.21 to $u^{\mathrm{e}}$ in $B_{R}^{+}$, and then conclude with the help of Lemma 2.9.

\section{Fractional harmonic MAPs AND Weighted harmonic MAPS With FreE Boundary}

In this section, our goal is to review in details the notion of weakly $s$-harmonic maps, the associated Euler-Lagrange equation, and more importantly to present its characterization in terms of fractional (nonlocal) conservation laws. We shall also prove at the end of this section that the fractional harmonic extension of an $s$-harmonic map satisfies a suitable (degenerate) partially free boundary condition, in the spirit of the classical harmonic map system with partially free boundary.

\subsection{Fractional harmonic maps into spheres and conservation laws.}

Definition 3.1. Let $\Omega \subseteq \mathbb{R}^{n}$ be a bounded open set. A map $u \in \widehat{H}^{s}\left(\Omega ; \mathbb{S}^{d-1}\right)$ is said to be a weakly s-harmonic map in $\Omega$ (with values in $\mathbb{S}^{d-1}$ ) if

$$
\left[\frac{\mathrm{d}}{\mathrm{d} t} \mathcal{E}_{s}\left(\frac{u+t \varphi}{|u+t \varphi|}, \Omega\right)\right]_{t=0}=0 \quad \forall \varphi \in \mathscr{D}\left(\Omega ; \mathbb{R}^{d}\right)
$$

If $u$ is also stationary in $\Omega$ (in the sense of Definition 2.16), we say that $u$ is a stationary weakly $s$-harmonic map in $\Omega$.

Definition 3.2. Let $\Omega \subseteq \mathbb{R}^{n}$ be a bounded open set. A map $u \in \widehat{H}^{s}\left(\Omega ; \mathbb{S}^{d-1}\right)$ is said to be a minimizing s-harmonic map in $\Omega$ (with values in $\mathbb{S}^{d-1}$ ) if

$$
\mathcal{E}_{s}(u, \Omega) \leqslant \mathcal{E}_{s}(w, \Omega)
$$

for every $w \in \widehat{H}^{s}\left(\Omega ; \mathbb{S}^{d-1}\right)$ such that $\operatorname{spt}(u-w)$ is compactly included in $\Omega$.

Remark 3.3. A minimizing $s$-harmonic map in $\Omega$ is obviously a critical point with respect to both inner and (constrained) outer variations of the energy. In other words, if $u$ is a minimizing $s$-harmonic map in $\Omega$, then $u$ is also a stationary weakly $s$-harmonic map in $\Omega$.

Remark 3.4. If $u \in \widehat{H}^{s}\left(\Omega ; \mathbb{S}^{d-1}\right)$ is a weakly $s$-harmonic map in $\Omega$ (stationary, minimizing, respectively), then $u$ is also weakly $s$-harmonic in $\Omega^{\prime}$ (stationary, minimizing, respectively) for any open subset $\Omega^{\prime} \subseteq \Omega$. It can be directly checked from the definitions, or one can rely on the Euler-Lagrange equation presented below and Remark 2.3.

Proposition 3.5. Let $\Omega \subseteq \mathbb{R}^{n}$ be a bounded open set. A map $u \in \widehat{H}^{s}\left(\Omega ; \mathbb{S}^{d-1}\right)$ is weakly s-harmonic in $\Omega$ if and only if

$$
\left\langle(-\Delta)^{s} u, \varphi\right\rangle_{\Omega}=0
$$

for every $\varphi \in H_{00}^{s}\left(\Omega ; \mathbb{R}^{d}\right)$ such that $\operatorname{spt}(\varphi) \subseteq \Omega$ and $\varphi(x) \in \operatorname{Tan}\left(u(x), \mathbb{S}^{d-1}\right)$ for a.e. $x \in \Omega$. Equivalently,

$$
(-\Delta)^{s} u(x)=\left(\frac{\gamma_{n, s}}{2} \int_{\mathbb{R}^{n}} \frac{|u(x)-u(y)|^{2}}{|x-y|^{n+2 s}} \mathrm{~d} y\right) u(x) \quad \text { in } \mathscr{D}^{\prime}(\Omega) .
$$

Proof. Let $u \in \widehat{H}^{s}\left(\Omega ; \mathbb{S}^{d-1}\right)$, fix $\varphi \in \mathscr{D}\left(\Omega ; \mathbb{R}^{d}\right)$, and notice that

$$
\left[\frac{\mathrm{d}}{\mathrm{d} t}\left(\frac{u+t \varphi}{|u+t \varphi|}\right)\right]_{t=0}=\varphi-(u \cdot \varphi) u \in H_{00}^{s}\left(\Omega ; \mathbb{R}^{d}\right) .
$$


Hence,

$$
\left[\frac{\mathrm{d}}{\mathrm{d} t} \mathcal{E}_{s}\left(\frac{u+t \varphi}{|u+t \varphi|}, \Omega\right)\right]_{t=0}=\left\langle(-\Delta)^{s} u, \varphi\right\rangle_{\Omega}-\left\langle(-\Delta)^{s} u,(u \cdot \varphi) u\right\rangle_{\Omega} .
$$

On the other hand, since $|u|^{2}=1$, we have

$$
\begin{aligned}
(u(x)-u(y)) \cdot((u(x) \cdot \varphi(x)) u(x) & -(u(y) \cdot \varphi(y)) u(y)) \\
& =\frac{1}{2}|u(x)-u(y)|^{2} u(x) \cdot \varphi(x)+\frac{1}{2}|u(x)-u(y)|^{2} u(y) \cdot \varphi(y),
\end{aligned}
$$

and it follows that

$$
\left\langle(-\Delta)^{s} u,(u \cdot \varphi) u\right\rangle_{\Omega}=\int_{\Omega}\left(\frac{\gamma_{n, s}}{2} \int_{\mathbb{R}^{n}} \frac{|u(x)-u(y)|^{2}}{|x-y|^{n+2 s}} \mathrm{~d} y\right) u(x) \cdot \varphi(x) \mathrm{d} x .
$$

Consequently, $u$ is weakly $s$-harmonic in $\Omega$ if and only if (3.2) holds.

By approximation, (3.2) also holds for any test function $\varphi \in H_{00}^{s}\left(\Omega ; \mathbb{R}^{d}\right) \cap L^{\infty}\left(\mathbb{R}^{n}\right)$ compactly supported in $\Omega$. In view of the right-hand side of (3.2), (3.1) clearly holds for every $\varphi \in$ $H_{00}^{s}\left(\Omega ; \mathbb{R}^{d}\right) \cap L^{\infty}\left(\mathbb{R}^{n}\right)$ compactly supported in $\Omega$ and satisfying $\varphi \cdot u=0$. By a standard truncation argument, it implies that (3.1) holds for every $\varphi \in H_{00}^{s}\left(\Omega ; \mathbb{R}^{d}\right)$ compactly supported in $\Omega$ and satisfying $\varphi \cdot u=0$.

The other way around, if (3.1) holds, then the map $\varphi-(u \cdot \varphi) u$ with $\varphi \in \mathscr{D}\left(\Omega ; \mathbb{R}^{d}\right)$ is admissible, and (3.1) combined with (3.3) shows that (3.2) holds, i.e., $u$ is weakly $s$-harmonic in $\Omega$.

Remark 3.6. The variational equation (3.1) corresponds to the weak formulation of the implicit equation

$$
(-\Delta)^{s} u \perp \operatorname{Tan}\left(u, \mathbb{S}^{d-1}\right) \text { in } \Omega,
$$

and in equation (3.2), the Lagrange multiplier associated with the $\mathbb{S}^{d-1}$-constraint is made explicit.

Remark 3.7. A weakly $s$-harmonic map $u$ in $\Omega$ which is smooth in $\Omega$, is stationary in $\Omega$. Indeed, if $X \in C^{1}\left(\Omega ; \mathbb{R}^{n}\right)$ is compactly supported in $\Omega$, the smoothness of $u$ implies that

$$
\delta \mathcal{E}_{s}(u, \Omega)[X]=\left\langle(-\Delta)^{s} u, X \cdot \nabla u\right\rangle_{\Omega} .
$$

Since $|u|^{2}=1$, we have $(X \cdot \nabla u) \cdot u=0$, and thus $\delta \mathcal{E}_{s}(u, \Omega)[X]=0$.

Now we rewrite the Euler-Lagrange equation (3.2) in a more compact form using the fractional $s$-gradient $\mathrm{d}_{s} u$ defined in Subsection 2.2. More precisely, if $u=:\left(u^{1}, \ldots, u^{d}\right)$, then

$$
\frac{\gamma_{n, s}}{2} \int_{\mathbb{R}^{n}} \frac{|u(x)-u(y)|^{2}}{|x-y|^{n+2 s}} \mathrm{~d} y=\sum_{j=1}^{d} \frac{\gamma_{n, s}}{2} \int_{\mathbb{R}^{n}} \frac{\left|u^{j}(x)-u^{j}(y)\right|^{2}}{|x-y|^{n+2 s}} \mathrm{~d} y=\sum_{j=1}^{d}\left|\mathrm{~d}_{s} u^{j}\right|^{2}=:\left|\mathrm{d}_{s} u\right|^{2},
$$

according to (2.4) and (2.5). We can thus rephrase Proposition 3.5 as follows: $u \in \widehat{H}^{s}\left(\Omega ; \mathbb{S}^{d-1}\right)$ is weakly $s$-harmonic in $\Omega$ if and only if

$$
(-\Delta)^{s} u=\left|\mathrm{d}_{s} u\right|^{2} u \text { in } \mathscr{D}^{\prime}(\Omega) .
$$

Our aim is to further rewrite equation (3.4), or more precisely its right-hand side, to reveal the fractional "div-curl structure" of Section 2.2 in the spirit of the well-known div-curl structure hidden in the classical equation for harmonic maps into spheres [24]. Following [29], the starting point is to notice that for each $i, j \in\{1, \ldots, d\}$,

$$
\begin{aligned}
&\left|\mathrm{d}_{s} u^{j}\right|^{2}(x) u^{i}(x)=\int_{\mathbb{R}^{n}} \frac{u^{i}(x) \mathrm{d}_{s} u^{j}(x, y) \mathrm{d}_{s} u^{j}(x, y)}{|x-y|^{n}} \mathrm{~d} y \\
&=\int_{\mathbb{R}^{n}} \frac{u^{i}(x) \mathrm{d}_{s} u^{j}(x, y)-u^{j}(x) \mathrm{d}_{s} u^{i}(x, y)}{|x-y|^{n}} \mathrm{~d}_{s} u^{j}(x, y) \mathrm{d} y \\
&+\left(\mathrm{d}_{s} u^{i} \odot \mathrm{d}_{s} u^{j}\right)(x) u^{j}(x) .
\end{aligned}
$$


Then, since $|u|^{2}=1$, we have

$$
\begin{aligned}
\sum_{j=1}^{d}\left(\mathrm{~d}_{s} u^{i} \odot \mathrm{d}_{s} u^{j}\right)(x) u^{j}(x) & =\sum_{j=1}^{d} \frac{\gamma_{n, s}}{2} \int_{\mathbb{R}^{n}} \frac{\left(u^{j}(x)-u^{j}(y)\right) u^{j}(x)}{|x-y|^{n+2 s}}\left(u^{i}(x)-u^{i}(y)\right) \mathrm{d} y \\
& =\frac{\gamma_{n, s}}{4} \int_{\mathbb{R}^{n}} \frac{|u(x)-u(y)|^{2}}{|x-y|^{n+2 s}}\left(u^{i}(x)-u^{i}(y)\right) \mathrm{d} y .
\end{aligned}
$$

We can now introduce for $i, j \in\{1, \ldots, d\}$,

$$
\boldsymbol{\Omega}^{i j}(x, y):=u^{i}(x) \mathrm{d}_{s} u^{j}(x, y)-u^{j}(x) \mathrm{d}_{s} u^{i}(x, y) \in L_{\text {od }}^{2}(\Omega),
$$

and

$$
T^{i}(x):=\frac{\gamma_{n, s}}{4} \int_{\mathbb{R}^{n}} \frac{|u(x)-u(y)|^{2}}{|x-y|^{n+2 s}}\left(u^{i}(x)-u^{i}(y)\right) \mathrm{d} y \in L^{1}(\Omega) .
$$

to derive from (3.5) and (3.6) the following reformulation of equation (3.4).

Lemma 3.8. Let $\Omega \subseteq \mathbb{R}^{n}$ be a bounded open set. A map $u \in \widehat{H}^{s}\left(\Omega ; \mathbb{S}^{d-1}\right)$ is weakly s-harmonic in $\Omega$ if and only if

$$
(-\Delta)^{s} u^{i}=\left(\sum_{j=1}^{d} \boldsymbol{\Omega}^{i j} \odot \mathrm{d}_{s} u^{j}\right)+T^{i} \quad \text { in } \mathscr{D}^{\prime}(\Omega)
$$

for every $i=1, \ldots, d$, where $\boldsymbol{\Omega}^{i j}$ and $T^{i}$ are given by (3.7) and (3.8), respectively.

Remark 3.9. The presence of the extra term $T^{i}$ in (3.9), compared the classical harmonic map equation (see [24]), is essentially due to the fact that the $s$-gradient $\mathrm{d}_{s} u$ is not tangent to the target sphere.

The fundamental observation made in [29, Lemma 3.1] for $\Omega=\mathbb{R}$ and $s=1 / 2$ is a characterization of the 1/2-harmonic map equation in terms of nonlocal conservation laws satisfied by the $\boldsymbol{\Omega}^{i j}$,s (thus extending [50] to the fractional setting). In the following proposition, we slightly generalize this result to a domain of arbitrary dimension and $s \in(0,1)$. The proof remains essentially the same, and we provide it for the reader's convenience.

Proposition 3.10. Let $\Omega \subseteq \mathbb{R}^{n}$ be a bounded open set with Lipschitz boundary. A map $u \in$ $\widehat{H}^{s}\left(\Omega ; \mathbb{S}^{d-1}\right)$ is weakly s-harmonic in $\Omega$ if and only if

$$
\operatorname{div}_{s} \boldsymbol{\Omega}^{i j}=0 \quad \text { in } H^{-s}(\Omega)
$$

for each $i, j \in\{1, \ldots, d\}$, where $\boldsymbol{\Omega}^{i j}$ is given by (3.7).

Proof. Step 1. Assume that $u$ is a weakly $s$-harmonic map in $\Omega$, and let us compute $\operatorname{div}_{s} \Omega^{i j}$. For $\varphi \in \mathscr{D}(\Omega)$, we have

$$
\begin{aligned}
& \int_{\mathbb{R}^{n}} \boldsymbol{\Omega}^{i j} \odot \mathrm{d}_{s} \varphi \mathrm{d} x= \\
& \quad \iint_{\left(\mathbb{R}^{n} \times \mathbb{R}^{n}\right) \backslash\left(\Omega^{c} \times \Omega^{c}\right)}\left(u^{i}(x) \mathrm{d}_{s} u^{j}(x, y) \mathrm{d}_{s} \varphi(x, y)-u^{j}(x) \mathrm{d}_{s} u^{i}(x, y) \mathrm{d}_{s} \varphi(x, y)\right) \frac{\mathrm{d} x \mathrm{~d} y}{|x-y|^{n}} .
\end{aligned}
$$

An elementary computation shows

$$
\left\{\begin{array}{l}
u^{i}(x) \mathrm{d}_{s} \varphi(x, y)=\mathrm{d}_{s}\left(u^{i} \varphi\right)(x, y)-\varphi(y) \mathrm{d}_{s} u^{i}(x, y) \\
u^{j}(x) \mathrm{d}_{s} \varphi(x, y)=\mathrm{d}_{s}\left(u^{j} \varphi\right)(x, y)-\varphi(y) \mathrm{d}_{s} u^{j}(x, y)
\end{array},\right.
$$

so that

$$
\int_{\mathbb{R}^{n}} \boldsymbol{\Omega}^{i j} \odot \mathrm{d}_{s} \varphi \mathrm{d} x=\int_{\mathbb{R}^{n}} \mathrm{~d}_{s} u^{j} \odot \mathrm{d}_{s}\left(u^{i} \varphi\right) \mathrm{d} x-\int_{\mathbb{R}^{n}} \mathrm{~d}_{s} u^{i} \odot \mathrm{d}_{s}\left(u^{j} \varphi\right) \mathrm{d} x .
$$

Since $u^{j} \varphi$ and $u^{i} \varphi$ belong to $H_{00}^{s}(\Omega)$, we infer from Proposition 2.4 and equation (3.4) that

$$
\begin{aligned}
\int_{\mathbb{R}^{n}} \Omega^{i j} \odot \mathrm{d}_{s} \varphi \mathrm{d} x & =\left\langle(-\Delta)^{s} u^{j}, u^{i} \varphi\right\rangle_{\Omega}-\left\langle(-\Delta)^{s} u^{i}, u^{j} \varphi\right\rangle_{\Omega} \\
& =\int_{\Omega}\left|\mathrm{d}_{s} u\right|^{2} u^{j} u^{i} \varphi \mathrm{d} x-\int_{\Omega}\left|\mathrm{d}_{s} u\right|^{2} u^{i} u^{j} \varphi \mathrm{d} x=0 .
\end{aligned}
$$

Therefore $\operatorname{div}_{s} \boldsymbol{\Omega}^{i j}=0$ in $\mathscr{D}^{\prime}(\Omega)$, and by approximation also in $H^{-s}(\Omega)$ (see $(2.2)$ ). 
Step 2. We assume that (3.10) holds, and we aim to prove that (3.4) holds. We fix $\varphi \in \mathscr{D}\left(\Omega ; \mathbb{R}^{d}\right)$, and we set $\psi:=\varphi-(u \cdot \varphi) u \in H_{00}^{s}\left(\Omega ; \mathbb{R}^{d}\right)$, which satisfies $\psi \cdot u=0$ a.e. in $\mathbb{R}^{n}$. As in the proof of Proposition 3.5, proving (3.4) reduces to show that

$$
\left\langle(-\Delta)^{s} u, \psi\right\rangle_{\Omega}=0 \text {. }
$$

Using $|u|^{2}=1$, we first observe that

$$
\left\langle(-\Delta)^{s} u, \psi\right\rangle_{\Omega}=\sum_{i=1}^{d}\left\langle(-\Delta)^{s} u^{i}, \psi^{i}\right\rangle_{\Omega}=\sum_{i, j=1}^{d}\left\langle(-\Delta)^{s} u^{i},\left(\psi^{i} u^{j}\right) u^{j}\right\rangle_{\Omega} .
$$

Since $\psi^{i} u^{j} \in H_{00}^{s}(\Omega)$, we obtain as in (3.11),

$$
\begin{aligned}
\left\langle(-\Delta)^{s} u^{i},\left(\psi^{i} u^{j}\right) u^{j}\right\rangle_{\Omega}=\left\langle(-\Delta)^{s} u^{j},\left(\psi^{i} u^{j}\right) u^{i}\right\rangle_{\Omega}-\int_{\mathbb{R}^{n}} \Omega^{i j} \odot \mathrm{d}_{s}\left(\psi^{i} u^{j}\right) \mathrm{d} x \\
=\left\langle(-\Delta)^{s} u^{j},\left(\psi^{i} u^{j}\right) u^{i}\right\rangle_{\Omega}
\end{aligned}
$$

for every $i, j \in\{1, \ldots, d\}$, thanks to (3.10). Therefore,

$$
\left\langle(-\Delta)^{s} u, \psi\right\rangle_{\Omega}=\sum_{i, j=1}^{d}\left\langle(-\Delta)^{s} u^{j},\left(\psi^{i} u^{j}\right) u^{i}\right\rangle_{\Omega}=\sum_{j=1}^{d}\left\langle(-\Delta)^{s} u^{j},(\psi \cdot u) u^{j}\right\rangle_{\Omega}=0,
$$

and the proof is complete.

\subsection{Weighted harmonic maps with free boundary.}

Definition 3.11. Let $G \subseteq \mathbb{R}_{+}^{n+1}$ be a bounded admissible open set, and $v \in H^{1}\left(G ; \mathbb{R}^{d},|z|^{a} \mathrm{~d} \mathbf{x}\right)$ satisfying $v(\mathbf{x}) \in \mathbb{S}^{d-1}$ for a.e. $\mathbf{x} \in \partial^{0} G$. The map $v$ is said to be a weighted weakly harmonic map in $G$ with respect to the partially free boundary condition $v\left(\partial^{0} G\right) \subseteq \mathbb{S}^{d-1}$ if

$$
\int_{G} z^{a} \nabla v \cdot \nabla \Phi \mathrm{d} \mathbf{x}=0
$$

for every $\Phi \in H^{1}\left(G ; \mathbb{R}^{d},|z|^{a} \mathrm{~d} \mathbf{x}\right)$ such that $\Phi=0$ on $\partial^{+} G$ and $\Phi(\mathbf{x}) \in \operatorname{Tan}\left(v(\mathbf{x}), \mathbb{S}^{d-1}\right)$ for a.e. $\mathbf{x} \in \partial^{0} G$. In short, we shall say that $v$ is a weighted weakly harmonic map with free boundary in $G$.

Remark 3.12. If $v \in H^{1}\left(G ; \mathbb{R}^{d},|z|^{a} \mathrm{~d} \mathbf{x}\right)$ is a weighted weakly harmonic map with free boundary in $G$, then (3.12) means that $v$ satisfies in the weak sense

$$
\begin{cases}\operatorname{div}\left(z^{a} \nabla v\right)=0 & \text { in } G \\ z^{a} \frac{\partial v}{\partial \nu} \perp \operatorname{Tan}\left(v, \mathbb{S}^{d-1}\right) & \text { on } \partial^{0} G .\end{cases}
$$

In particular, $v$ is smooth in $G$ by standard elliptic regularity.

In view of Remark 3.6, equation (3.13) above, and Lemma 2.12, it is clear that weighted weakly harmonic maps with free boundary and weakly $s$-harmonic maps are intimately related. This relation is made precise in the following proposition (see [32, Proposition 4.6]).

Proposition 3.13. Let $\Omega \subseteq \mathbb{R}^{n}$ be a bounded open set with Lipschitz boundary. If a map $u \in \widehat{H}^{s}\left(\Omega ; \mathbb{S}^{d-1}\right)$ is a weakly s-harmonic map in $\Omega$, then its extension $u^{\mathrm{e}}$ given by $(2.12)$ is a weighted weakly harmonic map with free boundary in every bounded admissible open set $G \subseteq \mathbb{R}_{+}^{n+1}$ satisfying $\overline{\partial^{0} G} \subseteq \Omega$.

Proof. Let us assume that $u$ is a weakly $s$-harmonic map in $\Omega$, and let $G \subseteq \mathbb{R}_{+}^{n+1}$ be bounded admissible open set such that $\overline{\partial^{0} G} \subseteq \Omega$. Let $\Phi \in H^{1}\left(G ; \mathbb{R}^{d},|z|^{a} \mathrm{dx}\right)$ such that $\Phi=0$ on $\partial^{+} G$, and $\Phi \cdot u=0$ on $\partial^{0} G$. We extend $\Phi$ by 0 to the whole half space $\mathbb{R}_{+}^{n+1}$, and the resulting map, still denoted by $\Phi$, belongs to $H^{1}\left(\mathbb{R}_{+}^{n+1} ; \mathbb{R}^{d},|z|^{a} \mathrm{~d} \mathbf{x}\right)$. In view of $(2.16), \Phi_{\mid \mathbb{R}^{n}} \in H_{00}^{s}\left(\Omega ; \mathbb{R}^{d}\right)$, and $\operatorname{spt}\left(\Phi_{\mid \mathbb{R}^{n}}\right) \subseteq \Omega$. Since $\Phi_{\mid \mathbb{R}^{n}} \cdot u=0$, we conclude from Lemma 2.12 and Proposition 3.5 that

$$
\int_{G} z^{a} \nabla u^{\mathrm{e}} \cdot \nabla \Phi \mathrm{d} \mathbf{x}=\int_{\mathbb{R}_{+}^{n+1}} z^{a} \nabla u^{\mathrm{e}} \cdot \nabla \Phi \mathrm{d} \mathbf{x}=\frac{1}{\boldsymbol{\delta}_{s}}\left\langle(-\Delta)^{s} u, \Phi_{\mid \mathbb{R}^{n}}\right\rangle_{\Omega}=0 .
$$

Hence, $u^{\mathrm{e}}$ is indeed a weighted weakly harmonic map with free boundary in $G$. 


\section{Small Energy HÖLder Regularity}

In this section, we present the main epsilon-regularity theorem asserting that under a certain smallness assumption of the energy in a ball, a weakly $s$-harmonic map satisfying the monotonicity formula is Hölder continuous in a smaller ball. Hölder regularity will be improved to Lipschitz regularity in the next section with an explicit control on the Lipschitz norm in terms of the energy.

Theorem 4.1. There exist constants $\varepsilon_{0}=\varepsilon_{0}(n, s)>0$ and $\beta_{0}=\beta_{0}(n, s) \in(0,1)$ such that the following holds. Let $u \in \widehat{H}^{s}\left(D_{R} ; \mathbb{S}^{d-1}\right)$ be a weakly s-harmonic map in $D_{R}$ such that the function $r \in(0, R-|\mathbf{x}|) \mapsto \boldsymbol{\Theta}_{s}\left(u^{\mathrm{e}}, \mathbf{x}, r\right)$ is non decreasing for every $\mathbf{x} \in \partial^{0} B_{R}^{+}$. If

$$
\boldsymbol{\theta}_{s}(u, 0, R) \leqslant \varepsilon_{0},
$$

then $u \in C^{0, \beta_{0}}\left(D_{R / 2}\right)$ and

$$
R^{2 \beta_{0}}[u]_{C^{0, \beta_{0}\left(D_{R / 2}\right)}}^{2} \leqslant C \boldsymbol{\theta}_{s}(u, 0, R),
$$

for a constant $C=C(n, s)$.

For what follows, it is useful to translate the epsilon-regularity theorem above only in terms of the extension. This is the purpose of the following corollary.

Corollary 4.2. There exist three constants $\varepsilon_{1}=\varepsilon_{1}(n, s)>0, \boldsymbol{\kappa}_{1}=\boldsymbol{\kappa}_{1}(n, s) \in(0,1), \beta_{1}=$ $\beta_{1}(n, s) \in(0,1)$ such that the following holds. Let $u \in \widehat{H}^{s}\left(D_{2 R} ; \mathbb{S}^{d-1}\right)$ be a weakly s-harmonic map in $D_{2 R}$ such that the function $r \in(0,2 R-|\mathbf{x}|) \mapsto \boldsymbol{\Theta}_{s}\left(u^{\mathrm{e}}, \mathbf{x}, r\right)$ is non decreasing for every $\mathbf{x} \in \partial^{0} B_{2 R}^{+}$. If

$$
\Theta_{s}\left(u^{\mathrm{e}}, 0, R\right) \leqslant \varepsilon_{1}
$$

then $u^{\mathrm{e}} \in C^{0, \beta_{1}}\left(B_{\kappa_{1} R}^{+}\right)$and

$$
R^{2 \beta_{1}}\left[u^{\mathrm{e}}\right]_{C^{0, \beta_{1}\left(B_{\kappa_{1} R}^{+}\right)}}^{2} \leqslant C
$$

for a constant $C=C(n, s)$.

Proof. We consider the constant $\varepsilon_{0}=\varepsilon_{0}(n, s)>0$ given by Theorem 4.1. Since $|u| \equiv 1$, we obtain from Lemma 2.19 the existence of $\varepsilon_{1}=\varepsilon_{1}(n, s)>0$ and $\alpha=\alpha(n, s) \in(0,1 / 4]$ such that the condition $\boldsymbol{\Theta}_{s}\left(u^{\mathrm{e}}, 0, R\right) \leqslant \varepsilon_{1}$ implies $\boldsymbol{\theta}_{s}(u, 0, \alpha R) \leqslant \boldsymbol{\varepsilon}_{0}$. In turn, Theorem 4.1 tells us that $u \in C^{0, \beta_{0}}\left(D_{\alpha R / 2}\right)$. Then Lemma 2.11 implies that $u^{\mathrm{e}} \in C^{0, \beta_{1}}\left(B_{\kappa_{1} R}^{+}\right)$with $\beta_{1}:=\min \left(\beta_{0}, s\right)$ and $\kappa_{1}:=\alpha / 8$. Moreover, combining (2.19) and (4.2) leads to

$$
\begin{aligned}
& R^{2 \beta_{1}}\left[u^{\mathrm{e}}\right]_{C^{0, \beta_{1}}\left(B_{\kappa_{1} R}^{+}\right)}^{2} \leqslant C\left(R^{2 \beta_{1}}[u]_{C^{0, \beta_{1}\left(D_{\alpha R / 2}\right)}}^{2}+1\right) \leqslant C\left(R^{2 \beta_{0}}[u]_{C^{0, \beta_{0}\left(D_{\alpha R / 2}\right)}}^{2}+1\right) \\
& \leqslant C\left(\boldsymbol{\theta}_{s}(u, 0, \alpha R)+1\right) \leqslant C,
\end{aligned}
$$

and the proof is complete.

Remark 4.3. In the case $n \leqslant 2 s$, the function $r \in(0, R-|\mathbf{x}|) \mapsto \boldsymbol{\Theta}_{s}\left(u^{\mathrm{e}}, \mathbf{x}, r\right)$ is nondecreasing for every $u \in \widehat{H}^{s}\left(D_{R} ; \mathbb{R}^{d}\right)$. In other words, in the case $n \leqslant 2 s$, Theorem 4.1 and Corollary 4.2 apply to arbitrary weakly $s$-harmonic maps. Moreover, in the case $n=1$ and $s \in(1 / 2,1)$ (i.e., $n<2 s$ ), the conclusions of Theorem 4.1 and Corollary 4.2 apply even without the smallness assumptions (4.1) or (4.3), since it follows from the classical imbedding $H^{s}(\mathbb{R}) \hookrightarrow C^{0, s-1 / 2}(\mathbb{R})$. For our purposes, it is convenient to state it suitably. This is the object of the proposition below, whose proof is postponed to the end of Section 4.1 .

Proposition 4.4. Assume that $n=1$ and $s \in(1 / 2,1)$. If $u \in \widehat{H}^{s}\left(D_{R} ; \mathbb{R}^{d}\right)$, then $u \in$ $C^{0, s-1 / 2}\left(D_{R / 2}\right)$ and

$$
R^{2 s-1}[u]_{C^{0, s-1 / 2}\left(D_{R / 2}\right)}^{2} \leqslant C \boldsymbol{\theta}_{s}(u, 0, R)
$$

for a constant $C=C(s)$. 
4.1. Proof of Theorem 4.1 and Proposition 4.4. The key point to prove Theorem 4.1 is to obtain a geometric decay of the energy in small balls. Then Hölder continuity follows classically from Campanato's criterion. The purpose of the next proposition, very much inspired from [17, Proposition 3.1], is exactly to show such decay.

Proposition 4.5. Assume that $n \geqslant 2 s$. There exist two constants $\varepsilon_{*}=\varepsilon_{*}(n, s)>0$ and $\boldsymbol{\tau}=\boldsymbol{\tau}(n, s) \in(0,1 / 4)$ such that the following holds. Let $u \in \widehat{H}^{s}\left(D_{1} ; \mathbb{S}^{d-1}\right)$ be a weakly $s$ harmonic map in $D_{1}$ such that the function $r \in(0,1-|\mathbf{x}|) \mapsto \boldsymbol{\Theta}_{s}\left(u^{\mathrm{e}}, \mathbf{x}, r\right)$ is non decreasing for every $\mathbf{x} \in \partial^{0} B_{1}^{+}$. If

$$
\mathcal{E}_{s}\left(u, D_{1}\right) \leqslant \varepsilon_{*},
$$

then

$$
\frac{1}{\boldsymbol{\tau}^{n-2 s}} \mathcal{E}_{s}\left(u, D_{\boldsymbol{\tau}}\right) \leqslant \frac{1}{2} \mathcal{E}_{s}\left(u, D_{1}\right) .
$$

Proof. We fix the constant $\tau \in(0,1 / 4)$ that will be specified later on. We proceed by contradiction assuming that there exists a sequence $\left\{u_{k}\right\}$ of weakly $s$-harmonic maps in $D_{1}$ such that $r \in(0,1-|\mathbf{x}|) \mapsto \boldsymbol{\Theta}_{s}\left(u_{k}^{\mathrm{e}}, \mathbf{x}, r\right)$ is non decreasing for every $\mathbf{x} \in \partial^{0} B_{1}^{+}$, and satisfying

$$
\varepsilon_{k}^{2}:=\mathcal{E}_{s}\left(u_{k}, D_{1}\right) \underset{k \rightarrow \infty}{\longrightarrow} 0
$$

and

$$
\frac{1}{\boldsymbol{\tau}^{n-2 s}} \mathcal{E}_{s}\left(u_{k}, D_{\boldsymbol{\tau}}\right)>\frac{1}{2} \mathcal{E}_{s}\left(u_{k}, D_{1}\right) .
$$

(Note that this later condition ensures that $\varepsilon_{k}>0$.) Then we consider the (expanded) map

$$
w_{k}:=\frac{u_{k}-\left(u_{k}\right)_{0,1}}{\varepsilon_{k}} \in \widehat{H}^{s}\left(D_{1} ; \mathbb{R}^{d}\right) \cap L^{\infty}\left(\mathbb{R}^{n}\right),
$$

which satisfies

$$
f_{D_{1}} w_{k} \mathrm{~d} x=0 \quad \text { and } \quad \mathcal{E}_{s}\left(w_{k}, D_{1}\right)=1 .
$$

Assumption (4.5) also rewrites

$$
\frac{1}{\boldsymbol{\tau}^{n-2 s}} \mathcal{E}_{s}\left(w_{k}, D_{\boldsymbol{\tau}}\right)>\frac{1}{2}
$$

By Poincaré's inequality in $H^{s}\left(D_{1}\right)$, we have

$$
\left\|w_{k}\right\|_{L^{2}\left(D_{1}\right)}^{2} \leqslant C \mathcal{E}_{s}\left(w_{k}, D_{1}\right) \leqslant C .
$$

Therefore $\left\{w_{k}\right\}$ is bounded in $\widehat{H}^{s}\left(D_{1} ; \mathbb{R}^{d}\right)$, so that we can find a (not relabeled) subsequence and $w \in \widehat{H}^{s}\left(D_{1} ; \mathbb{R}^{d}\right)$ such that $w_{k} \rightarrow w$ weakly in $\widehat{H}^{s}\left(D_{1}\right)$ and $w_{k} \rightarrow w$ strongly in $L^{2}\left(D_{1}\right)$ (see Remark 2.2). In particular, $\|w\|_{L^{2}\left(D_{1}\right)} \leqslant C$. By lower semicontinuity of the energy $\mathcal{E}_{s}\left(\cdot, D_{1}\right)$, we also have $\mathcal{E}_{s}\left(w, D_{1}\right) \leqslant 1$ (see again Remark 2.2).

Recalling that $u_{k}$ satisfies

$$
\left\langle(-\Delta)^{s} u_{k}, \varphi\right\rangle_{D_{1}}=\int_{D_{1}}\left|\mathrm{~d}_{s} u_{k}\right|^{2} u_{k} \cdot \varphi \mathrm{d} x \quad \forall \varphi \in \mathscr{D}\left(D_{1} ; \mathbb{R}^{d}\right),
$$

we obtain in terms of $w_{k}$,

$$
\left\langle(-\Delta)^{s} w_{k}, \varphi\right\rangle_{D_{1}}=\varepsilon_{k} \int_{D_{1}}\left|\mathrm{~d}_{s} w_{k}\right|^{2} u_{k} \cdot \varphi \mathrm{d} x \quad \forall \varphi \in \mathscr{D}\left(D_{1} ; \mathbb{R}^{d}\right) .
$$

Since $\left|u_{k}\right| \equiv 1$, it leads to

$$
\begin{aligned}
\left|\left\langle(-\Delta)^{s} w_{k}, \varphi\right\rangle_{D_{1}}\right| \leqslant \varepsilon_{k}\left\|\left|\mathrm{~d}_{s} w_{k}\right|^{2}\right\|_{L^{1}\left(D_{1}\right)} & \|\varphi\|_{L^{\infty}\left(D_{1}\right)} \\
& \leqslant 2 \varepsilon_{k} \mathcal{E}_{s}\left(w_{k}, D_{1}\right)\|\varphi\|_{L^{\infty}\left(D_{1}\right)}=2 \varepsilon_{k}\|\varphi\|_{L^{\infty}\left(D_{1}\right)} \underset{k \rightarrow \infty}{\longrightarrow} 0
\end{aligned}
$$

for every $\varphi \in \mathscr{D}\left(D_{1} ; \mathbb{R}^{d}\right)$. On the other hand, the weak convergence in $\widehat{H}^{s}\left(D_{1}\right)$ of $w_{k}$ towards $w$ implies that

$$
\left\langle(-\Delta)^{s} w_{k}, \varphi\right\rangle_{D_{1}} \underset{k \rightarrow \infty}{\longrightarrow}\left\langle(-\Delta)^{s} w, \varphi\right\rangle_{D_{1}} \quad \forall \varphi \in \mathscr{D}\left(D_{1} ; \mathbb{R}^{d}\right) .
$$

As a consequence, $w$ satisfies

$$
(-\Delta)^{s} w=0 \quad \text { in } H^{-s}\left(D_{1}\right)
$$


By Lemma B.1 in Appendix B, $w$ is (locally) smooth in $D_{1}$, and we have the estimate

$$
\|w\|_{L^{\infty}\left(D_{1 / 2}\right)}^{2}+\|\nabla w\|_{L^{\infty}\left(D_{1 / 2}\right)}^{2} \leqslant C\left(\mathcal{E}_{s}\left(w, D_{1}\right)+\|w\|_{L^{2}\left(D_{1}\right)}^{2}\right) \leqslant C .
$$

In view of (4.9), we have

$$
\iint_{D_{\boldsymbol{\tau}} \times D_{\boldsymbol{\tau}}} \frac{|w(x)-w(y)|^{2}}{|x-y|^{n+2 s}} \mathrm{~d} x \mathrm{~d} y \leqslant C \iint_{D_{\boldsymbol{\tau}} \times D_{\boldsymbol{\tau}}} \frac{\mathrm{d} x \mathrm{~d} y}{|x-y|^{n+2 s-2}} \leqslant C \boldsymbol{\tau}^{n+2-2 s} .
$$

Then, writing

$$
\begin{aligned}
\iint_{D_{\boldsymbol{\tau}} \times D_{\tau}^{c}} \frac{|w(x)-w(y)|^{2}}{|x-y|^{n+2 s}} \mathrm{~d} x \mathrm{~d} y=\iint_{D_{\boldsymbol{\tau}} \times\left(D_{1 / 2} \backslash D_{\boldsymbol{\tau}}\right)} \frac{|w(x)-w(y)|^{2}}{|x-y|^{n+2 s}} \mathrm{~d} x \mathrm{~d} y \\
+\iint_{D_{\boldsymbol{\tau}} \times D_{1 / 2}^{c}} \frac{|w(x)-w(y)|^{2}}{|x-y|^{n+2 s}} \mathrm{~d} x \mathrm{~d} y
\end{aligned}
$$

we first estimate, using (4.9),

$$
\iint_{D_{\boldsymbol{\tau}} \times\left(D_{1 / 2} \backslash D_{\boldsymbol{\tau}}\right)} \frac{|w(x)-w(y)|^{2}}{|x-y|^{n+2 s}} \mathrm{~d} x \mathrm{~d} y \leqslant C \iint_{D_{\boldsymbol{\tau}} \times\left(D_{1 / 2} \backslash D_{\boldsymbol{\tau}}\right)} \frac{\mathrm{d} x \mathrm{~d} y}{|x-y|^{n+2 s-2}} \leqslant C \boldsymbol{\tau}^{n} .
$$

Next we infer from Lemma 2.1 and (4.9) that

$$
\begin{aligned}
& \iint_{D_{\boldsymbol{\tau}} \times D_{1 / 2}^{c}} \frac{|w(x)-w(y)|^{2}}{|x-y|^{n+2 s}} \mathrm{~d} x \mathrm{~d} y \leqslant 2 \iint_{D_{\boldsymbol{\tau}} \times D_{1 / 2}^{c}} \frac{|w(x)|^{2}+|w(y)|^{2}}{|x-y|^{n+2 s}} \mathrm{~d} x \mathrm{~d} y \\
& \leqslant C\left(\int_{D_{\boldsymbol{\tau}}}|w(x)|^{2} \mathrm{~d} x+\boldsymbol{\tau}^{n} \int_{D_{1 / 2}^{c}} \frac{|w(y)|^{2}}{(|y|+1)^{n+2 s}} \mathrm{~d} y\right) \leqslant C \boldsymbol{\tau}^{n} .
\end{aligned}
$$

Gathering (4.10), (4.11), (4.12), and (4.13) yields

$$
\frac{1}{\boldsymbol{\tau}^{n-2 s}} \mathcal{E}_{s}\left(w, D_{\boldsymbol{\tau}}\right) \leqslant C \boldsymbol{\tau}^{2 s} .
$$

By Lemma 4.6 - which is postponed at the end of the proof - there exists a universal constant $\boldsymbol{\sigma} \in(0,1)$ such that

$$
w_{k} \rightarrow w \text { strongly in } H^{s}\left(D_{\boldsymbol{\sigma}}\right) .
$$

In view of (4.14), we can choose $\tau$ (depending only on $n$ and $s$ ) in such a way that

$$
0<\boldsymbol{\tau}<\boldsymbol{\sigma} / 2 \text { and } \frac{1}{\boldsymbol{\tau}^{n-2 s}} \mathcal{E}_{s}\left(w, D_{\boldsymbol{\tau}}\right) \leqslant \frac{1}{4}
$$

From (4.10) and the strong convergence in (4.15), we first infer that for $k$ large enough,

$$
\iint_{D_{\boldsymbol{\tau}} \times D_{\boldsymbol{\tau}}} \frac{\left|w_{k}(x)-w_{k}(y)\right|^{2}}{|x-y|^{n+2 s}} \mathrm{~d} x \mathrm{~d} y \leqslant \iint_{D_{\boldsymbol{\tau}} \times D_{\boldsymbol{\tau}}} \frac{|w(x)-w(y)|^{2}}{|x-y|^{n+2 s}} \mathrm{~d} x \mathrm{~d} y+\boldsymbol{\tau}^{n} .
$$

In the same way, for $k$ large enough, one obtains from (4.15),

$$
\begin{aligned}
\iint_{D_{\boldsymbol{\tau}} \times D_{\boldsymbol{\tau}}^{c}} \frac{\left|w_{k}(x)-w_{k}(y)\right|^{2}}{|x-y|^{n+2 s}} \mathrm{~d} x \mathrm{~d} y & =\iint_{D_{\boldsymbol{\tau}} \times\left(D_{\boldsymbol{\sigma}} \backslash D_{\boldsymbol{\tau}}\right)} \frac{\left|w_{k}(x)-w_{k}(y)\right|^{2}}{|x-y|^{n+2 s}} \mathrm{~d} x \mathrm{~d} y \\
& +\iint_{D_{\boldsymbol{\tau}} \times D_{\boldsymbol{\sigma}}^{c}} \frac{\left|w_{k}(x)-w_{k}(y)\right|^{2}}{|x-y|^{n+2 s}} \mathrm{~d} x \mathrm{~d} y \\
\leqslant \boldsymbol{\tau}^{n}+\iint_{D_{\boldsymbol{\tau}} \times\left(D_{\boldsymbol{\sigma}} \backslash D_{\boldsymbol{\tau}}\right)} & \frac{|w(x)-w(y)|^{2}}{|x-y|^{n+2 s}} \mathrm{~d} x \mathrm{~d} y \\
& +\iint_{D_{\boldsymbol{\tau}} \times D_{\boldsymbol{\sigma}}^{c}} \frac{\left|w_{k}(x)-w_{k}(y)\right|^{2}}{|x-y|^{n+2 s}} \mathrm{~d} x \mathrm{~d} y .
\end{aligned}
$$

Then we estimate by means of Lemma 2.1,

$$
\begin{aligned}
\iint_{D_{\boldsymbol{\tau}} \times D_{\boldsymbol{\sigma}}^{c}} & \frac{\left|w_{k}(x)-w_{k}(y)\right|^{2}}{|x-y|^{n+2 s}} \mathrm{~d} x \mathrm{~d} y \leqslant 2 \iint_{D_{\boldsymbol{\tau}} \times D_{\boldsymbol{\sigma}}^{c}} \frac{\left|w_{k}(x)\right|^{2}+\left|w_{k}(y)\right|^{2}}{|x-y|^{n+2 s}} \mathrm{~d} x \mathrm{~d} y \\
& \leqslant C\left(\int_{D_{\boldsymbol{\tau}}}\left|w_{k}(x)\right|^{2} \mathrm{~d} x+\boldsymbol{\tau}^{n} \int_{D_{\boldsymbol{\sigma}}^{c}} \frac{\left|w_{k}(y)\right|^{2}}{(|y|+1)^{n+2 s}} \mathrm{~d} y\right) \leqslant C\left(\int_{D_{\boldsymbol{\tau}}}\left|w_{k}(x)\right|^{2} \mathrm{~d} x+\boldsymbol{\tau}^{n}\right) .
\end{aligned}
$$


Since $w_{k} \rightarrow w$ strongly in $L^{2}\left(D_{1}\right)$ and in view of (4.9), we deduce that for $k$ large enough,

$$
\iint_{D_{\boldsymbol{\tau}} \times D_{\boldsymbol{\sigma}}^{c}} \frac{\left|w_{k}(x)-w_{k}(y)\right|^{2}}{|x-y|^{n+2 s}} \mathrm{~d} x \mathrm{~d} y \leqslant C\left(\int_{D_{\boldsymbol{\tau}}}|w(x)|^{2} \mathrm{~d} x+\boldsymbol{\tau}^{n}\right) \leqslant C \boldsymbol{\tau}^{n} .
$$

Combining (4.17), (4.18), and (4.19) together with (4.16), we conclude that for $k$ large enough,

$$
\frac{1}{\boldsymbol{\tau}^{n-2 s}} \mathcal{E}_{s}\left(w_{k}, D_{\boldsymbol{\tau}}\right) \leqslant \frac{1}{\boldsymbol{\tau}^{n-2 s}} \mathcal{E}_{s}\left(w, D_{\boldsymbol{\tau}}\right)+C \boldsymbol{\tau}^{2 s} \leqslant \frac{1}{4}+C \boldsymbol{\tau}^{2 s} .
$$

Hence, we can choose $\boldsymbol{\tau} \in(0,1 / 4)$ small enough (depending only on $n$ and $s)$ in such a way that $\frac{1}{\tau^{n-2 s}} \mathcal{E}_{s}\left(w_{k}, D_{\boldsymbol{\tau}}\right) \leqslant 1 / 2$ whenever $k$ is large enough, contradicting (4.6).

As it is transparent from the proof above, Proposition 4.5 crucially rests on the strong convergence stated in (4.15) that we now prove.

Lemma 4.6. There exists a universal constant $\boldsymbol{\sigma} \in(0,1)$ such that the weakly converging subsequence $\left\{w_{k}\right\}$ (towards $w$ ) actually converges strongly in $H^{s}\left(D_{\boldsymbol{\sigma}}\right)$.

Proof. We choose the constant $\boldsymbol{\sigma}$ as follows:

$$
\sigma:=\min \left\{\frac{4}{5 \Lambda}, \frac{1}{32}\right\}
$$

where $\Lambda>1$ is the universal constant given by Theorem 2.5 .

Step 1. Subtracting (4.8) from equation (4.7) leads to

$$
\left\langle(-\Delta)^{s}\left(w_{k}-w\right), \varphi\right\rangle_{D_{1}}=\varepsilon_{k} \int_{D_{1}}\left|\mathrm{~d}_{s} w_{k}\right|^{2} u_{k} \cdot \varphi \mathrm{d} x \quad \forall \varphi \in \mathscr{D}\left(D_{1} ; \mathbb{R}^{d}\right) .
$$

By approximation (see (2.2)), this equation also holds for every $\varphi \in H_{00}^{s}\left(D_{1} ; \mathbb{R}^{d}\right) \cap L^{\infty}\left(D_{1}\right)$ compactly supported in $D_{1}$. Let us now fix a smooth cut-off function $\zeta \in \mathscr{D}\left(D_{5 \sigma / 4}\right)$ such that $0 \leqslant \zeta \leqslant 1, \zeta=1$ in $D_{\sigma}$. Using the test function $\varphi_{k}:=\zeta\left(w_{k}-w\right) \in H_{00}^{s}\left(D_{1} ; \mathbb{R}^{d}\right) \cap L^{\infty}\left(D_{1}\right)$ in $(4.20)$ yields

$$
\left\langle(-\Delta)^{s}\left(w_{k}-w\right), \varphi_{k}\right\rangle_{D_{1}}=\varepsilon_{k} \int_{D_{1}}\left|\mathrm{~d}_{s} w_{k}\right|^{2} u_{k} \cdot \varphi_{k} \mathrm{~d} x
$$

Setting

$$
L_{k}:=\left\langle(-\Delta)^{s}\left(w_{k}-w\right), \zeta\left(w_{k}-w\right)\right\rangle_{D_{1}} \quad \text { and } \quad R_{k}:=\varepsilon_{k} \int_{D_{1}}\left|\mathrm{~d}_{s} w_{k}\right|^{2} u_{k} \cdot \varphi_{k} \mathrm{~d} x
$$

we claim that

$$
L_{k} \geqslant\left[w_{k}-w\right]_{H^{s}\left(D_{\boldsymbol{\sigma}}\right)}^{2}+o(1) \quad \text { as } k \rightarrow \infty,
$$

and

$$
\lim _{k \rightarrow \infty} R_{k}=0 .
$$

Identity (4.21) rewrites $L_{k}=R_{k}$, and the two claims above will imply that $\left[w_{k}-w\right]_{H^{s}\left(D_{\sigma}\right)}^{2} \rightarrow 0$ as $k \rightarrow \infty$, whence the conclusion.

Step 2. This step is devoted to the proof of (4.22). For simplicity, let us denote

$$
\triangle_{k}:=w_{k}-w .
$$

Since $\zeta=1$ in $D_{\boldsymbol{\sigma}}$, and $\zeta=0$ in $D_{2 \boldsymbol{\sigma}}^{c}$, we have

$$
L_{k}=\left[\triangle_{k}\right]_{H^{s}\left(D_{\boldsymbol{\sigma}}\right)}^{2}+\frac{\gamma_{n, s}}{2}\left(L_{k}^{(1)}+L_{k}^{(2)}+L_{k}^{(3)}\right),
$$

with

and

$$
\begin{aligned}
L_{k}^{(1)} & :=\iint_{\left(D_{1} \backslash D_{\boldsymbol{\sigma}}\right) \times\left(D_{1} \backslash D_{\boldsymbol{\sigma}}\right)} \frac{\left(\triangle_{k}(x)-\triangle_{k}(y)\right) \cdot\left(\zeta(x) \triangle_{k}(x)-\zeta(y) \triangle_{k}(y)\right)}{|x-y|^{n+2 s}} \mathrm{~d} x \mathrm{~d} y, \\
L_{k}^{(2)} & :=2 \iint_{D_{\boldsymbol{\sigma}} \times\left(D_{1} \backslash D_{\boldsymbol{\sigma}}\right)} \frac{\left(\triangle_{k}(x)-\triangle_{k}(y)\right) \cdot\left(\zeta(x) \triangle_{k}(x)-\zeta(y) \triangle_{k}(y)\right)}{|x-y|^{n+2 s}} \mathrm{~d} x \mathrm{~d} y,
\end{aligned}
$$

$$
L_{k}^{(3)}:=2 \iint_{D_{2 \sigma} \times D_{1}^{c}} \frac{\left(\triangle_{k}(x)-\triangle_{k}(y)\right) \cdot \triangle_{k}(x)}{|x-y|^{n+2 s}} \zeta(x) \mathrm{d} x \mathrm{~d} y,
$$


Concerning $L_{k}^{(1)}$, we first rewrite

$$
\begin{aligned}
& L_{k}^{(1)}=\iint_{\left(D_{1} \backslash D_{\boldsymbol{\sigma}}\right) \times\left(D_{1} \backslash D_{\boldsymbol{\sigma}}\right)} \frac{\left(\left(\triangle_{k}(x)-\triangle_{k}(y)\right) \cdot \triangle_{k}(x)\right)(\zeta(x)-\zeta(y))}{|x-y|^{n+2 s}} \mathrm{~d} x \mathrm{~d} y \\
& \quad+\iint_{\left(D_{1} \backslash D_{\boldsymbol{\sigma}}\right) \times\left(D_{1} \backslash D_{\boldsymbol{\sigma}}\right)} \frac{\left|\triangle_{k}(x)-\triangle_{k}(y)\right|^{2}}{|x-y|^{n+2 s}} \zeta(y) \mathrm{d} x \mathrm{~d} y \\
& \geqslant \iint_{\left(D_{1} \backslash D_{\boldsymbol{\sigma}}\right) \times\left(D_{1} \backslash D_{\boldsymbol{\sigma}}\right)} \frac{\left(\left(\triangle_{k}(x)-\triangle_{k}(y)\right) \cdot \triangle_{k}(x)\right)(\zeta(x)-\zeta(y))}{|x-y|^{n+2 s}} \mathrm{~d} x \mathrm{~d} y .
\end{aligned}
$$

Recalling that

$$
\mathcal{E}_{s}\left(\triangle_{k}, D_{1}\right) \leqslant 2 \mathcal{E}_{s}\left(w_{k}, D_{1}\right)+2 \mathcal{E}_{s}\left(w, D_{1}\right) \leqslant 4,
$$

we estimate by means of Hölder's inequality,

$$
\begin{gathered}
\left|\iint_{\left(D_{1} \backslash D_{\sigma}\right) \times\left(D_{1} \backslash D_{\sigma}\right)} \frac{\left(\left(\triangle_{k}(x)-\triangle_{k}(y)\right) \cdot \triangle_{k}(x)\right)(\zeta(x)-\zeta(y))}{|x-y|^{n+2 s}} \mathrm{~d} x \mathrm{~d} y\right| \\
\leqslant C \sqrt{\mathcal{E}_{s}\left(\triangle_{k}, D_{1}\right)}\left(\iint_{\left(D_{1} \backslash D_{\sigma}\right) \times\left(D_{1} \backslash D_{\sigma}\right)} \frac{\left|\triangle_{k}(x)\right|^{2}|\zeta(x)-\zeta(y)|^{2}}{|x-y|^{n+2 s}} \mathrm{~d} x \mathrm{~d} y\right)^{1 / 2} \\
\leqslant C\left(\iint_{\left(D_{1} \backslash D_{\boldsymbol{\sigma}}\right) \times\left(D_{1} \backslash D_{\boldsymbol{\sigma}}\right)} \frac{\left|\triangle_{k}(x)\right|^{2}}{|x-y|^{n+2 s-2}} \mathrm{~d} x \mathrm{~d} y\right)^{1 / 2} \leqslant C\left\|\triangle_{k}\right\|_{L^{2}\left(D_{1}\right)} .
\end{gathered}
$$

Since $\left\|\triangle_{k}\right\|_{L^{2}\left(D_{1}\right)} \rightarrow 0$, we conclude that

$$
L_{k}^{(1)} \geqslant o(1) \quad \text { as } k \rightarrow \infty .
$$

Exactly in the same way, one derives

$$
L_{k}^{(2)} \geqslant o(1) \quad \text { as } k \rightarrow \infty
$$

For the last term $L_{k}^{(3)}$, we use again Hölder's inequality to derive

$$
\left|L_{k}^{(3)}\right| \leqslant C \sqrt{\mathcal{E}_{s}\left(\triangle_{k}, D_{1}\right)}\left(\iint_{D_{2 \sigma} \times D_{1}^{c}} \frac{\left|\triangle_{k}(x)\right|^{2} \zeta^{2}(x)}{|x-y|^{n+2 s}} \mathrm{~d} x \mathrm{~d} y\right)^{1 / 2} \leqslant C\left\|\triangle_{k}\right\|_{L^{2}\left(D_{1}\right)}=o(1)
$$

as $k \rightarrow \infty$. Gathering now (4.24) with (4.25), (4.26), and (4.27) leads to (4.22).

Step 3. In order to prove (4.23), we need to rewrite $R_{k}$ in a suitable form. First, we rewrite

$$
R_{k}=\frac{1}{\varepsilon_{k}} \int_{D_{1}}\left|\mathrm{~d}_{s} u_{k}\right|^{2} u_{k} \cdot \varphi_{k} \mathrm{~d} x,
$$

and we recall from Lemma 3.8 that for each $i=1, \ldots, d$,

$$
\left|\mathrm{d}_{s} u_{k}\right|^{2} u_{k}^{i}=\left(\sum_{j=1}^{d} \boldsymbol{\Omega}_{k}^{i j} \odot \mathrm{d}_{s} u_{k}^{j}\right)+T_{k}^{i}=\varepsilon_{k}\left(\sum_{j=1}^{d} \boldsymbol{\Omega}_{k}^{i j} \odot \mathrm{d}_{s} w_{k}^{j}\right)+T_{k}^{i},
$$

where $\boldsymbol{\Omega}_{k}^{i j} \in L_{\text {od }}^{2}\left(D_{1}\right)$ is given by

$$
\boldsymbol{\Omega}_{k}^{i j}(x, y):=u_{k}^{i}(x) \mathrm{d}_{s} u_{k}^{j}(x, y)-u_{k}^{j}(x) \mathrm{d}_{s} u_{k}^{i}(x, y),
$$

and

$$
\begin{aligned}
T_{k}^{i}(x) & :=\frac{\gamma_{n, s}}{4} \int_{\mathbb{R}^{n}} \frac{\left|u_{k}(x)-u_{k}(y)\right|^{2}}{|x-y|^{n+2 s}}\left(u_{k}^{i}(x)-u_{k}^{i}(y)\right) \mathrm{d} y \\
& =\frac{\gamma_{n, s} \varepsilon_{k}^{3}}{4} \int_{\mathbb{R}^{n}} \frac{\left|w_{k}(x)-w_{k}(y)\right|^{2}}{|x-y|^{n+2 s}}\left(w_{k}^{i}(x)-w_{k}^{i}(y)\right) \mathrm{d} y .
\end{aligned}
$$

Hence,

$$
R_{k}=\left(\sum_{i, j=1}^{d} \int_{D_{1}}\left(\Omega_{k}^{i j} \odot \mathrm{d}_{s} w_{k}^{j}\right) \varphi_{k}^{i} \mathrm{~d} x\right)+\varepsilon_{k}^{2} \int_{D_{1}} \widetilde{T}_{k} \cdot \varphi_{k} \mathrm{~d} x=: R_{k}^{(1)}+R_{k}^{(2)},
$$


where we have set

$$
\widetilde{T}_{k}(x):=\frac{\gamma_{n, s}}{4} \int_{\mathbb{R}^{n}} \frac{\left|w_{k}(x)-w_{k}(y)\right|^{2}}{|x-y|^{n+2 s}}\left(w_{k}(x)-w_{k}(y)\right) \mathrm{d} y .
$$

Step 4. We shall now prove that

$$
\lim _{k \rightarrow \infty} R_{k}^{(1)}=0
$$

First, notice that formula (2.12) shows that $u_{k}^{\mathrm{e}}=\varepsilon_{k} w_{k}^{\mathrm{e}}+\left(u_{k}\right)_{0,1}$, which implies that

$$
\boldsymbol{\Theta}_{s}\left(u_{k}^{\mathrm{e}}, \mathbf{x}, r\right)=\varepsilon_{k}^{2} \boldsymbol{\Theta}_{s}\left(w_{k}^{\mathrm{e}}, \mathbf{x}, r\right) \quad \text { for every } \mathbf{x} \in \partial^{0} B_{1}^{+} \text {and } r \in(0,1-|\mathbf{x}|) .
$$

As a consequence, our assumption on $\boldsymbol{\Theta}_{s}\left(u_{k}^{\mathrm{e}}, \mathbf{x}, r\right)$ tells us that $r \in(0,1-|\mathbf{x}|) \mapsto \boldsymbol{\Theta}_{s}\left(w_{k}^{\mathrm{e}}, \mathbf{x}, r\right)$ is non decreasing for every $\mathbf{x} \in \partial^{0} B_{1}^{+}$.

Applying Corollary 2.23 (with $R=2 \boldsymbol{\sigma}$ ), we deduce that

$$
\left[\zeta w_{k}\right]_{\mathrm{BMO}\left(\mathbb{R}^{n}\right)} \leqslant C\left(\mathcal{E}_{s}\left(w_{k}, 4 \boldsymbol{\sigma}\right)+\left\|w_{k}\right\|_{L^{2}\left(D_{4 \sigma}\right)}^{2}\right)^{1 / 2} \leqslant C
$$

for some constant $C$ depending only on $n, s$, and $\zeta$. Since $w_{k} \rightarrow w$ strongly in $L^{2}\left(D_{1}\right)$ and $\zeta$ is supported in $D_{5 \boldsymbol{\sigma} / 4}$, we have $\zeta w_{k} \rightarrow \zeta w$ strongly in $L^{1}\left(\mathbb{R}^{n}\right)$ (in other words, $\left\|\varphi_{k}\right\|_{L^{1}\left(\mathbb{R}^{n}\right)} \rightarrow 0$ ). By lower semi-continuity of the BMO-seminorm with respect to the $L^{1}$-convergence, we deduce that $\zeta w \in \operatorname{BMO}\left(\mathbb{R}^{n}\right)$, and then (remember that $\varphi_{k}:=\zeta\left(w_{k}-w\right)$ )

$$
\left[\varphi_{k}\right]_{\mathrm{BMO}\left(\mathbb{R}^{n}\right)} \leqslant C .
$$

Next, we recall from Proposition 3.10 that $u_{k}$ being weakly $s$-harmonic in $D_{1}$ yields

$$
\operatorname{div}_{s} \boldsymbol{\Omega}_{k}^{i j}=0 \quad \text { in } H^{-s}\left(D_{1}\right)
$$

for each $i, j \in\{1, \ldots, d\}$. Applying Theorem 2.5 (with $x_{0}=0$ and $r=5 \boldsymbol{\sigma} / 4$ ), we infer that

$$
\begin{aligned}
\left|\int_{D_{1}}\left(\boldsymbol{\Omega}_{k}^{i j} \odot \mathrm{d}_{s} w_{k}^{j}\right) \varphi_{k}^{i} \mathrm{~d} x\right| & \leqslant C\left\|\boldsymbol{\Omega}_{k}^{i j}\right\|_{L_{\mathrm{od}}^{2}\left(D_{1}\right)} \sqrt{\mathcal{E}_{s}\left(w_{k}^{j}, D_{1}\right)}\left(\left[\varphi_{k}^{i}\right]_{\mathrm{BMO}\left(\mathbb{R}^{n}\right)}+\left\|\varphi_{k}^{i}\right\|_{L^{1}\left(\mathbb{R}^{n}\right)}\right) \\
& \leqslant C\left\|\boldsymbol{\Omega}_{k}^{i j}\right\|_{L_{\mathrm{od}}^{2}\left(D_{1}\right)} .
\end{aligned}
$$

Since $\left|u_{k}\right| \equiv 1$, we have the pointwise estimate $\left|\boldsymbol{\Omega}_{k}^{i j}(x, y)\right| \leqslant\left|\mathrm{d}_{s} u_{k}^{j}(x, y)\right|+\left|\mathrm{d}_{s} u_{k}^{i}(x, y)\right|$ which leads to $\left\|\boldsymbol{\Omega}_{k}^{i j}\right\|_{L_{\text {od }}^{2}\left(D_{1}\right)}^{2} \leqslant C \mathcal{E}_{s}\left(u_{k}, D_{1}\right)=O\left(\varepsilon_{k}^{2}\right)$ for each $i, j \in\{1, \ldots, d\}$. Consequently,

$$
R_{k}^{(1)}=O\left(\varepsilon_{k}\right)
$$

and (4.28) is proved.

Step 5. We complete the proof of (4.23) showing now that

$$
\lim _{k \rightarrow \infty} R_{k}^{(2)}=0
$$

Using the fact that $\varphi_{k}$ is supported in $D_{5 \sigma / 4} \subseteq D_{1 / 20} \subseteq D_{1 / 16}$, we first write

$$
R_{k}^{(2)}=\varepsilon_{k}^{2} \int_{D_{1}} \widetilde{T}_{k} \cdot \varphi_{k} \mathrm{~d} x=\frac{\gamma_{n, s}}{4} \varepsilon_{k}^{2}\left(I_{k}+I I_{k}\right),
$$

with

$$
\begin{aligned}
I_{k} & :=\iint_{D_{1 / 16} \times D_{1 / 16}} \frac{\left|w_{k}(x)-w_{k}(y)\right|^{2}}{|x-y|^{n+2 s}}\left(w_{k}(x)-w_{k}(y)\right) \cdot \varphi_{k}(x) \mathrm{d} x \mathrm{~d} y \\
& =\frac{1}{2} \iint_{D_{1 / 16} \times D_{1 / 16}} \frac{\left|w_{k}(x)-w_{k}(y)\right|^{2}}{|x-y|^{n+2 s}}\left(w_{k}(x)-w_{k}(y)\right) \cdot\left(\varphi_{k}(x)-\varphi_{k}(y)\right) \mathrm{d} x \mathrm{~d} y,
\end{aligned}
$$

and

$$
I I_{k}:=\iint_{D_{1 / 20} \times D_{1 / 16}^{c}} \frac{\left|w_{k}(x)-w_{k}(y)\right|^{2}}{|x-y|^{n+2 s}}\left(w_{k}(x)-w_{k}(y)\right) \cdot \varphi_{k}(x) \mathrm{d} x \mathrm{~d} y .
$$

We shall estimate separately the two terms $I_{k}$ and $I I_{k}$. 
Concerning $I_{k}$, we apply Hölder's inequality to reach

$$
\begin{aligned}
\left|I_{k}\right| & \leqslant \frac{1}{2} \iint_{D_{1 / 16} \times D_{1 / 16}} \frac{\left|w_{k}(x)-w_{k}(y)\right|^{3}\left|\varphi_{k}(x)-\varphi_{k}(y)\right|}{|x-y|^{n+2 s}} \mathrm{~d} x \mathrm{~d} y \\
& \leqslant C\left[w_{k}\right]_{W^{s / 3,6}\left(D_{1 / 16}\right)}^{3}\left[\varphi_{k}\right]_{H^{s}\left(D_{1 / 16}\right)},
\end{aligned}
$$

where $[\cdot]_{W^{s / 3,6}\left(D_{1 / 16}\right)}$ denotes the $W^{s / 3,6}\left(D_{1 / 16}\right)$-seminorm (i.e., of the Sobolev-Slobodeckij space, see (C.3)). Recalling our notation $\triangle_{k}:=w_{k}-w$ and the fact that $0 \leqslant \zeta \leqslant 1$, we have

$$
\begin{aligned}
{\left[\varphi_{k}\right]_{H^{s}\left(D_{1 / 16}\right)}^{2} } & \leqslant C\left(\left[\triangle_{k}\right]_{H^{s}\left(D_{1 / 16}\right)}^{2}+\iint_{D_{1 / 16} \times D_{1 / 16}} \frac{|\zeta(x)-\zeta(y)|^{2}\left|\triangle_{k}(x)\right|^{2}}{|x-y|^{n+2 s}} \mathrm{~d} x \mathrm{~d} y\right) \\
& \leqslant C\left(\left[\triangle_{k}\right]_{H^{s}\left(D_{1 / 16}\right)}^{2}+\iint_{D_{1 / 16} \times D_{1 / 16}} \frac{\left|\triangle_{k}(x)\right|^{2}}{|x-y|^{n+2 s-2}} \mathrm{~d} x \mathrm{~d} y\right) \\
& \leqslant C\left(\mathcal{E}_{s}\left(\triangle_{k}, D_{1 / 16}\right)+\left\|\triangle_{k}\right\|_{L^{2}\left(D_{1 / 16}\right)}^{2}\right) \leqslant C .
\end{aligned}
$$

To estimate $\left[w_{k}\right]_{W^{s / 3,6}\left(D_{1 / 16}\right)}$, we proceed as follows. First, we fix a further cut-off function $\eta \in \mathscr{D}\left(D_{1 / 8}\right)$ satisfying $0 \leqslant \eta \leqslant 1, \eta \equiv 1$ in $D_{1 / 16}$, and $|\nabla \eta| \leqslant C$. Then we apply Corollary C.6 (in Appendix C) to $\eta w_{k}$ to derive

$$
\left[w_{k}\right]_{W^{s / 3,6}\left(D_{1 / 16}\right)}^{2}=\left[\eta w_{k}\right]_{W^{s / 3,6}\left(D_{1 / 16}\right)}^{2} \leqslant C\left(\sup _{D_{r}(\bar{x}) \subseteq \mathbb{R}^{n}} \frac{1}{r^{n-2 s}}\left[\eta w_{k}\right]_{H^{s}\left(D_{r}(\bar{x})\right)}^{2}\right),
$$

and it remains to estimate the right hand side of (4.34). To this purpose, we need to distinguish different types of balls:

Case 1: $\bar{x} \in D_{3 / 16}$ and $0<r \leqslant 1 / 32$. Arguing as in (4.33), we obtain

$$
\begin{aligned}
{\left[\eta w_{k}\right]_{H^{s}\left(D_{r}(\bar{x})\right)}^{2} } & \leqslant C\left(\left[w_{k}\right]_{H^{s}\left(D_{r}(\bar{x})\right)}^{2}+\iint_{D_{r}(\bar{x}) \times D_{r}(\bar{x})} \frac{\left|w_{k}(x)\right|^{2}}{|x-y|^{n+2 s-2}} \mathrm{~d} x \mathrm{~d} y\right) \\
& \leqslant C\left(\left[w_{k}\right]_{H^{s}\left(D_{r}(\bar{x})\right)}^{2}+r^{2-2 s}\left\|w_{k}\right\|_{L^{2}\left(D_{r}(\bar{x})\right)}^{2}\right) .
\end{aligned}
$$

Applying Hölder's inequality in the case $n \geqslant 3$, we obtain

$$
\left[\eta w_{k}\right]_{H^{s}\left(D_{r}(\bar{x})\right)}^{2} \leqslant \begin{cases}C\left(\left[w_{k}\right]_{H^{s}\left(D_{r}(\bar{x})\right)}^{2}+r^{n-2 s}\left\|w_{k}\right\|_{L^{n}\left(D_{r}(\bar{x})\right)}^{2}\right) & \text { if } n \geqslant 3 \\ C\left(\left[w_{k}\right]_{H^{s}\left(D_{r}(\bar{x})\right)}^{2}+r^{2-2 s}\left\|w_{k}\right\|_{L^{2}\left(D_{r}(\bar{x})\right)}^{2}\right) & \text { if } n \leqslant 2 .\end{cases}
$$

Let us now recall that $r \mapsto \boldsymbol{\Theta}_{s}\left(w_{k}^{\mathrm{e}}, \mathbf{x}, r\right)$ is non decreasing for every $\mathbf{x} \in B_{1}^{+}$(see Step 4). By the proof of Lemma 2.21, Step 1 (applied to $w_{k}^{\mathrm{e}}$ ), we have

$$
\left[w_{k}\right]_{\mathrm{BMO}\left(D_{7 / 32}\right)} \leqslant C \sqrt{\mathbf{E}_{s}\left(w_{k}^{\mathrm{e}}, B_{1 / 4}^{+}\right)} \leqslant C \sqrt{\mathcal{E}_{s}\left(w_{k}, D_{1}\right)} \leqslant C,
$$

where we have used Lemma 2.9 in the last inequality. In case $n \geqslant 3$, we apply the JohnNirenberg inequality in Lemma 2.22 and use the fact that $D_{r}(\bar{x}) \subseteq D_{7 / 32}$, to derive

$$
\begin{aligned}
\left\|w_{k}\right\|_{L^{n}\left(D_{r}(\bar{x})\right)} \leqslant\left\|w_{k}\right\|_{L^{n}\left(D_{7 / 32}\right)} \leqslant \| w_{k}- & \left(w_{k}\right)_{0,7 / 32}\left\|_{L^{n}\left(D_{7 / 32}\right)}+C\right\| w_{k} \|_{L^{1}\left(D_{7 / 32}\right)} \\
& \leqslant C\left(\left[w_{k}\right]_{\mathrm{BMO}\left(D_{7 / 32}\right)}+\left\|w_{k}\right\|_{L^{2}\left(D_{7 / 32}\right)}\right) \leqslant C .
\end{aligned}
$$

Back to (4.35) and in view of Lemma 2.8, we have thus proved that

$$
\begin{aligned}
& {\left[\eta w_{k}\right]_{H^{s}\left(D_{r}(\bar{x})\right)}^{2} \leqslant C\left(\left[w_{k}\right]_{H^{s}\left(D_{r}(\bar{x})\right)}^{2}+r^{n-2 s}\right)} \\
& \quad \leqslant C\left(\mathbf{E}_{s}\left(w_{k}^{\mathrm{e}}, B_{2 r}^{+}(\overline{\mathbf{x}})\right)+r^{n-2 s}\right) \leqslant C r^{n-2 s}\left(\boldsymbol{\Theta}_{s}\left(w_{k}^{\mathrm{e}}, \overline{\mathbf{x}}, 2 r\right)+1\right),
\end{aligned}
$$

with $\overline{\mathbf{x}}:=(\bar{x}, 0)$. Then the monotonicity of $r \mapsto \boldsymbol{\Theta}_{s}\left(w_{k}^{\mathrm{e}}, \overline{\mathbf{x}}, 2 r\right)$ together with Lemma 2.9 yields

$$
\begin{aligned}
\frac{1}{r^{n-2 s}}\left[\eta w_{k}\right]_{H^{s}\left(D_{r}(\bar{x})\right)}^{2} \leqslant C\left(\boldsymbol{\Theta}_{s}\left(w_{k}^{\mathrm{e}}, \overline{\mathbf{x}}, 1 / 16\right)+1\right) & \\
& \leqslant C\left(\mathbf{E}_{s}\left(w_{k}^{\mathrm{e}}, B_{1 / 4}^{+}\right)+1\right) \leqslant C\left(\mathcal{E}_{s}\left(w_{k}, D_{1}\right)+1\right) \leqslant C .
\end{aligned}
$$

Case 2: $\bar{x} \notin D_{3 / 16}$ and $0<r \leqslant 1 / 32$. This case is trivial since $\eta w_{k} \equiv 0$ in $D_{r}(\bar{x})$. 
Case 3: $\bar{x} \in \mathbb{R}^{n}$ and $r>1 / 32$. Since $\eta w_{k}$ is supported in $D_{1 / 8}$ and $0 \leqslant \eta \leqslant 1$, we have (recall that $n-2 s \geqslant 0)$

$$
\begin{aligned}
\frac{1}{r^{n-2 s}}\left[\eta w_{k}\right]_{H^{s}\left(D_{r}(\bar{x})\right)}^{2} & \leqslant 32^{2 s-n}\left[\eta w_{k}\right]_{H^{s}\left(\mathbb{R}^{n}\right)}^{2} \\
& \leqslant C\left(\left[\eta w_{k}\right]_{H^{s}\left(D_{1 / 4}\right)}^{2}+\iint_{D_{1 / 8} \times D_{1 / 4}^{c}} \frac{\left|\eta(x) w_{k}(x)\right|^{2}}{|x-y|^{n+2 s}} \mathrm{~d} x \mathrm{~d} y\right) \\
& \leqslant C\left(\left[\eta w_{k}\right]_{H^{s}\left(D_{1 / 4}\right)}^{2}+\left\|w_{k}\right\|_{L^{2}\left(D_{1 / 8}\right)}^{2}\right) .
\end{aligned}
$$

Arguing as in (4.33), we obtain

and thus

$$
\left[\eta w_{k}\right]_{H^{s}\left(D_{1 / 4}\right)}^{2} \leqslant C\left(\mathcal{E}_{s}\left(w_{k}, D_{1 / 4}\right)+\left\|w_{k}\right\|_{L^{2}\left(D_{1 / 4}\right)}^{2}\right)
$$

$$
\frac{1}{r^{n-2 s}}\left[\eta w_{k}\right]_{H^{s}\left(D_{r}(\bar{x})\right)}^{2} \leqslant C\left(\mathcal{E}_{s}\left(w_{k}, D_{1}\right)+\left\|w_{k}\right\|_{L^{2}\left(D_{1}\right)}^{2}\right) \leqslant C
$$

Gathering Cases 1, 2, and 3 above, we have proved that the right hand side of (4.34) remains bounded independently of $k$. We can now conclude from $(4.34)$ that $\left[w_{k}\right]_{W^{s / 3,6}\left(D_{1 / 16}\right)} \leqslant C$. In view of (4.32) and (4.33), we have thus obtained that

$$
\left|I_{k}\right| \leqslant C
$$

and it only remains to estimate the term $I I_{k}$ (defined in (4.31)).

First, we trivially have

$$
\begin{aligned}
\left|I I_{k}\right| & \leqslant \iint_{D_{1 / 20} \times D_{1 / 16}^{c}} \frac{\left|w_{k}(x)-w_{k}(y)\right|^{3}}{|x-y|^{n+2 s}}\left|\triangle_{k}(x)\right| \mathrm{d} x \mathrm{~d} y \\
& \leqslant 4 \iint_{D_{1 / 20} \times D_{1 / 16}^{c}} \frac{\left|w_{k}(x)\right|^{3}}{|x-y|^{n+2 s}}\left|\triangle_{k}(x)\right| \mathrm{d} x \mathrm{~d} y \\
& \quad+4 \iint_{D_{1 / 20} \times D_{1 / 16}^{c}} \frac{\left|w_{k}(y)\right|^{3}}{|x-y|^{n+2 s}}\left|\triangle_{k}(x)\right| \mathrm{d} x \mathrm{~d} y .
\end{aligned}
$$

On the other hand,

$$
\begin{aligned}
\iint_{D_{1 / 20} \times D_{1 / 16}^{c}} \frac{\left|w_{k}(x)\right|^{3}}{|x-y|^{n+2 s}}\left|\triangle_{k}(x)\right| \mathrm{d} x \mathrm{~d} y \leqslant C \int_{D_{1 / 20}}\left|w_{k}(x)\right|^{3}\left|\triangle_{k}(x)\right| \mathrm{d} x & \leqslant C\left\|w_{k}\right\|_{L^{6}\left(D_{1 / 20}\right)}^{3}\left\|\triangle_{k}\right\|_{L^{2}\left(D_{1}\right)} .
\end{aligned}
$$

Recalling from (4.36) that $\left\{w_{k}\right\}$ is bounded in $\operatorname{BMO}\left(D_{7 / 16}\right)$, we can argue as in (4.37) to infer that $\left\{w_{k}\right\}$ is bounded in $L^{6}\left(D_{1 / 20}\right)$. Hence,

$$
\iint_{D_{1 / 20} \times D_{1 / 16}^{c}} \frac{\left|w_{k}(x)\right|^{3}}{|x-y|^{n+2 s}}\left|\triangle_{k}(x)\right| \mathrm{d} x \mathrm{~d} y \leqslant C\left\|\triangle_{k}\right\|_{L^{2}\left(D_{1}\right)} .
$$

Since $\left|u_{k}\right| \equiv 1$, we have $\left|w_{k}\right| \leqslant 2 / \varepsilon_{k}$, and consequently

$$
\begin{aligned}
\iint_{D_{1 / 20} \times D_{1 / 16}^{c}} \frac{\left|w_{k}(y)\right|^{3}}{|x-y|^{n+2 s}}\left|\triangle_{k}(x)\right| \mathrm{d} x \mathrm{~d} y & \leqslant \frac{2}{\varepsilon_{k}} \int_{D_{1 / 20}}\left(\int_{D_{1 / 16}^{c}} \frac{\left|w_{k}(y)\right|^{2}}{|x-y|^{n+2 s}} \mathrm{~d} y\right)\left|\triangle_{k}(x)\right| \mathrm{d} x \\
& \leqslant \frac{C}{\varepsilon_{k}} \int_{D_{1 / 20}}\left(\int_{\mathbb{R}^{n}} \frac{\left|w_{k}(y)\right|^{2}}{(|y|+1)^{n+2 s}} \mathrm{~d} y\right)\left|\triangle_{k}(x)\right| \mathrm{d} x \\
& \leqslant \frac{C}{\varepsilon_{k}}\left(\mathcal{E}_{s}\left(w_{k}, D_{1}\right)+\left\|w_{k}\right\|_{L^{2}\left(D_{1}\right)}^{2}\right)\left\|\triangle_{k}\right\|_{L^{2}\left(D_{1}\right)}
\end{aligned}
$$

where we have used Lemma 2.1 in the last inequality. Combining (4.40), (4.41), and (4.42), we obtain the estimate

$$
\left|I I_{k}\right| \leqslant C \varepsilon_{k}^{-1}\left\|\triangle_{k}\right\|_{L^{2}\left(D_{1}\right)}=o\left(\varepsilon_{k}^{-1}\right) .
$$

In view of $(4.30),(4.39)$, and (4.43), we have thus proved that

$$
R_{k}^{(2)}=o\left(\varepsilon_{k}\right),
$$

and thus (4.29) holds, which completes the whole proof. 
Proof of Theorem 4.1. Rescaling variables, we can assume that $R=2$. We need to distinguish the two cases $n \geqslant 2 s$, and $n=1$ with $s \in(1 / 2,1)$.

Case 1: $n \geqslant 2 s$. We choose $\varepsilon_{0}:=2^{2 s-n} \varepsilon_{*}$ where $\varepsilon_{*}=\varepsilon_{*}(n, s)>0$ is the constant provided by Proposition 4.5. We fix an arbitrary point $x_{0} \in D_{1}$, and we observe that condition (4.1) implies

$$
\mathcal{E}_{s}\left(u, D_{1}\left(x_{0}\right)\right) \leqslant \mathcal{E}_{s}\left(u, D_{2}\right)=2^{n-2 s} \boldsymbol{\theta}_{s}(u, 0,2) \leqslant \boldsymbol{\varepsilon}_{*} .
$$

Setting $\mathbf{e}:=\mathcal{E}_{s}\left(u, D_{2}\right)$, Proposition 4.5 then leads to

$$
\frac{1}{\boldsymbol{\tau}^{n-2 s}} \mathcal{E}_{s}\left(u, D_{\boldsymbol{\tau}}\left(x_{0}\right)\right) \leqslant \frac{1}{2} \mathcal{E}_{s}\left(u, D_{1}\left(x_{0}\right)\right) \leqslant \frac{1}{2} \mathbf{e},
$$

where $\boldsymbol{\tau}=\boldsymbol{\tau}(n, s) \in(0,1 / 4)$. Considering the rescaled map $u_{\boldsymbol{\tau}}(x):=u\left(\boldsymbol{\tau} x+x_{0}\right)$, one realizes from (4.44) that $u_{\boldsymbol{\tau}}$ satisfies $\mathcal{E}_{s}\left(u_{\boldsymbol{\tau}}, D_{1}\right) \leqslant \frac{1}{2} \varepsilon_{*}$, and thus Proposition 4.5 applies. Unscaling variables, it yields

$$
\frac{1}{\left(\boldsymbol{\tau}^{n-2 s}\right)^{2}} \mathcal{E}_{s}\left(u, D_{\boldsymbol{\tau}^{2}}\left(x_{0}\right)\right)=\frac{1}{\boldsymbol{\tau}^{n-2 s}} \mathcal{E}_{s}\left(u_{\boldsymbol{\tau}}, D_{\boldsymbol{\tau}}\right) \leqslant \frac{1}{2} \mathcal{E}_{s}\left(u_{\boldsymbol{\tau}}, D_{1}\right)=\frac{1}{2 \boldsymbol{\tau}^{n-2 s}} \mathcal{E}_{s}\left(u, D_{\boldsymbol{\tau}}\left(x_{0}\right)\right) \leqslant \frac{1}{4} \mathbf{e}
$$

Arguing by induction, we infer that

$$
\mathcal{E}_{s}\left(u, D_{\boldsymbol{\tau}^{k}}\left(x_{0}\right)\right) \leqslant \frac{\boldsymbol{\tau}^{k(n-2 s)}}{2^{k}} \mathbf{e} \quad \text { for each } k=0,1,2,3, \ldots
$$

Let us now fix an arbitrary $r \in(0,1)$, and consider the integer $k$ such that $\tau^{k+1}<r \leqslant \tau^{k}$. From (4.45), we deduce that

$$
\frac{1}{r^{n-2 s}} \mathcal{E}_{s}\left(u, D_{r}\left(x_{0}\right)\right) \leqslant \frac{1}{r^{n-2 s}} \mathcal{E}_{s}\left(u, D_{\tau^{k}}\left(x_{0}\right)\right) \leqslant \frac{\tau^{2 s-n}}{2^{k}} \mathbf{e} \leqslant 2 \tau^{2 s-n} \mathbf{e} r^{2 \beta_{0}},
$$

with $2 \beta_{0}:=\log (2) / \log (1 / \tau)$. By the Poincaré inequality in $H^{s}\left(D_{r}\left(x_{0}\right)\right)$, it yields

$$
\frac{1}{r^{n}} \int_{D_{r}\left(x_{0}\right)}\left|u-(u)_{x_{0}, r}\right|^{2} \mathrm{~d} x \leqslant \frac{C}{r^{n-2 s}}[u]_{H^{s}\left(D_{r}\left(x_{0}\right)\right)}^{2} \leqslant \frac{C}{r^{n-2 s}} \mathcal{E}_{s}\left(u, D_{r}\left(x_{0}\right)\right) \leqslant C \mathbf{e} r^{2 \beta_{0}} .
$$

In view of the arbitrariness of $r$ and $x_{0}$, we can apply Campanato's criterion (see e.g. [27, Theorem I.6.1]), and it yields $u \in C^{0, \beta_{0}}\left(D_{1}\right)$ with

$$
|u(x)-u(y)| \leqslant C \sqrt{\mathbf{e}}|x-y|^{\beta_{0}} \quad \forall x, y \in D_{1},
$$

which completes the proof.

Case 2: $n=1$ and $s \in(1 / 2,1)$. In this case, we simply choose $\varepsilon_{0}:=1$, and we invoke Proposition 4.4 whose proof is given below.

Proof of Proposition 4.4. Rescaling variables, we can assume that $R=1$. Without loss of generality, we can also assume that $u$ has a vanishing average over $D_{1}$. We consider a given cut-off function $\zeta \in \mathscr{D}\left(D_{3 / 4}\right)$ such that $0 \leqslant \zeta \leqslant 1$ and $\zeta=1$ in $D_{1 / 2}$. Arguing as (4.38), we obtain that $\zeta u \in H^{s}\left(\mathbb{R} ; \mathbb{R}^{d}\right)$ with

$$
[\zeta u]_{H^{s}(\mathbb{R})}^{2} \leqslant C\left(\mathcal{E}_{s}\left(u, D_{1}\right)+\|u\|_{L^{2}\left(D_{1}\right)}^{2}\right) .
$$

On the other hand, by the continuous embedding $H^{s}\left(\mathbb{R}^{n}\right) \hookrightarrow C^{0, s-1 / 2}\left(\mathbb{R}^{n}\right)$ (see e.g. [20, Theorem 1.4.4.1]), we have

$$
[\zeta u]_{C^{0, s-1 / 2}(\mathbb{R})}^{2} \leqslant C\left([\zeta u]_{H^{s}(\mathbb{R})}^{2}+\|\zeta u\|_{L^{2}(\mathbb{R})}^{2}\right) \leqslant C\left([\zeta u]_{H^{s}(\mathbb{R})}^{2}+\|u\|_{L^{2}\left(D_{1}\right)}^{2}\right) .
$$

Combining (4.47) with (4.46) and applying Poincaré's inequality in $H^{s}\left(D_{1}\right)$, we derive that

$$
[u]_{C^{0, s-1 / 2}\left(D_{1 / 2}\right)}^{2} \leqslant[\zeta u]_{C^{0, s-1 / 2}(\mathbb{R})}^{2} \leqslant C\left(\mathcal{E}_{s}\left(u, D_{1}\right)+\|u\|_{L^{2}\left(D_{1}\right)}^{2}\right) \leqslant C \mathcal{E}_{s}\left(u, D_{1}\right),
$$

which completes the proof of (4.4). 


\section{Small Energy Lipschitz Regularity}

In this section, our goal is to improve the conclusion of Theorem 4.1 to Lipschitz continuity, as stated in the following theorem. Higher order regularity will be the object of the next section.

Theorem 5.1. Let $\varepsilon_{1}=\varepsilon_{1}(n, s)>0$ be the constant given by Corollary 4.2. There exists a constant $\kappa_{2}=\kappa_{2}(n, s) \in(0,1)$ such that the following holds. Let $u \in \widehat{H}^{s}\left(D_{2 R} ; \mathbb{S}^{d-1}\right)$ be a weakly s-harmonic map in $D_{2 R}$ such that the function $r \in(0,2 R-|\mathbf{x}|) \mapsto \boldsymbol{\Theta}_{s}\left(u^{\mathrm{e}}, \mathbf{x}, r\right)$ is nondecreasing for every $\mathbf{x} \in \partial^{0} B_{2 R}^{+}$. If

$$
\Theta_{s}\left(u^{\mathrm{e}}, 0, R\right) \leqslant \varepsilon_{1}
$$

then $u \in C^{0,1}\left(D_{\kappa_{2} R}\right)$ and

$$
R^{2}\|\nabla u\|_{L^{\infty}\left(D_{\kappa_{2} R}\right)}^{2} \leqslant C \boldsymbol{\Theta}_{s}\left(u^{\mathrm{e}}, 0, R\right),
$$

for a constant $C=C(n, s)$.

The proof of Theorem 5.1 consists in considering the system satisfied by the $\mathbb{S}^{d-1}$-valued map $u^{\mathrm{e}} /\left|u^{\mathrm{e}}\right|$. By Corollary $4.2, u^{\mathrm{e}}$ is Hölder continuous, and therefore $\left|u^{\mathrm{e}}\right| \geqslant 1 / 2$ in a smaller half ball $B_{r}^{+}$. In particular, $v:=u^{\mathrm{e}} /\left|u^{\mathrm{e}}\right|$ is well defined and Hölder continuous in $B_{r}^{+}$. We shall see that it satisfies in the weak sense the degenerate system with homogeneous Neumann boundary condition

$$
\begin{cases}-\operatorname{div}\left(z^{a} \rho^{2} \nabla v\right)=z^{a} \rho^{2}|\nabla v|^{2} v & \text { in } B_{r}^{+}, \\ z^{a} \rho^{2} \frac{\partial v}{\partial \nu}=0 & \text { on } \partial^{0} B_{r}^{+},\end{cases}
$$

with Hölder continuous weight $\rho^{2}:=\left|u^{\mathrm{e}}\right|^{2}$. Up to the extra weight term $\rho^{2}$, this system fits into the class of degenerate harmonic map systems with free boundary considered in [40]. Adjusting the arguments in [40] to take care of the extra weight $\rho^{2}$, we shall prove that $v$ is Lipschitz continuous in an even smaller half ball. Since $u^{\mathrm{e}}=v$ on $\partial^{0} B_{r}^{+}$, the conclusion will follow straight away.

5.1. Proof of Theorem 5.1. The aforementioned Lipschitz estimate on the map $u^{\mathrm{e}} /\left|u^{\mathrm{e}}\right|$ is the object of the following proposition.

Proposition 5.2. Let $u \in \widehat{H}^{s}\left(D_{2 R} ; \mathbb{S}^{d-1}\right)$ be a weakly s-harmonic map in $D_{2 R}$. Assume that $u^{\mathrm{e}} \in C^{0, \beta}\left(B_{R}^{+}\right)$for some exponent $\beta \in(0,1)$, and that $\left|u^{\mathrm{e}}\right| \geqslant 1 / 2$ in $B_{R}^{+}$. Setting $\eta:=$ $R^{\beta}\left[u^{\mathrm{e}}\right]_{C^{0, \beta}\left(B_{R}^{+}\right)}$, the map $u^{\mathrm{e}} /\left|u^{\mathrm{e}}\right|$ is Lipschitz continuous in $\bar{B}_{R / 3}^{+}$, and

$$
R^{2}\left\|\nabla\left(u^{\mathrm{e}} /\left|u^{\mathrm{e}}\right|\right)\right\|_{L^{\infty}\left(B_{R / 3}^{+}\right)}^{2} \leqslant C_{\eta, \beta} \Theta_{s}\left(u^{\mathrm{e}}, 0, R\right),
$$

for a constant $C_{\eta, \beta}=C_{\eta, \beta}(\eta, \beta, n, s)$.

Before proving this proposition, we need to show that $u^{\mathrm{e}} /\left|u^{\mathrm{e}}\right|$ satisfies system (5.2) in the weak sense.

Lemma 5.3. Let $u \in \widehat{H}^{s}\left(D_{2 R} ; \mathbb{S}^{d-1}\right)$ be a weakly $s$-harmonic map in $D_{2 R}$. Assume that $\rho:=\left|u^{\mathrm{e}}\right|$ satisfies $\rho \geqslant 1 / 2$ a.e. in $B_{R}^{+}$. Then the map $v:=u^{\mathrm{e}} / \rho$ belongs to $H^{1}\left(B_{R}^{+} ; \mathbb{R}^{d},|z|^{a} \mathrm{~d} \mathbf{x}\right)$ and it satisfies

$$
\int_{B_{R}^{+}} z^{a} \rho^{2} \nabla v \cdot \nabla \phi \mathrm{d} \mathbf{x}=\int_{B_{R}^{+}} z^{a} \rho^{2}|\nabla v|^{2} v \cdot \phi \mathrm{d} \mathbf{x}
$$

for every $\phi \in H^{1}\left(B_{R}^{+} ; \mathbb{R}^{d},|z|^{a} \mathrm{~d} \mathbf{x}\right) \cap L^{\infty}\left(B_{R}^{+}\right)$such that $\phi=0$ on $\partial^{+} B_{R}$

Proof. First recall from $(2.14)$ and Lemma 2.9 that $u^{\mathrm{e}} \in H^{1}\left(B_{R}^{+} ; \mathbb{R}^{d},|z|^{a} \mathrm{~d} \mathbf{x}\right) \cap L^{\infty}\left(\mathbb{R}_{+}^{n+1}\right)$, and consequently, $\rho \in H^{1}\left(B_{R}^{+},|z|^{a} \mathrm{~d} \mathbf{x}\right) \cap L^{\infty}\left(\mathbb{R}_{+}^{n+1}\right)$. By assumption $\rho \geqslant 1 / 2$, so that $1 / \rho \in$ $H^{1}\left(B_{R}^{+},|z|^{a} \mathrm{~d} \mathbf{x}\right) \cap L^{\infty}\left(\mathbb{R}_{+}^{n+1}\right)$. The space $H^{1}\left(B_{R}^{+},|z|^{a} \mathrm{~d} \mathbf{x}\right) \cap L^{\infty}\left(\mathbb{R}_{+}^{n+1}\right)$ being an algebra, it follows that $v \in H^{1}\left(B_{R}^{+} ; \mathbb{R}^{d},|z|^{a} \mathrm{~d} \mathbf{x}\right)$, and by definition $|v|=1$ a.e. in $B_{R}^{+}$.

Let us now fix $\Phi \in H^{1}\left(B_{R}^{+} ; \mathbb{R}^{d},|z|^{a} \mathrm{~d} \mathbf{x}\right) \cap L^{\infty}\left(B_{R}^{+}\right)$such that $\Phi=0$ on $\partial^{+} B_{R}$. Again, $H^{1}\left(B_{R}^{+} ; \mathbb{R}^{d},|z|^{a} \mathrm{~d} \mathbf{x}\right) \cap L^{\infty}\left(B_{R}^{+}\right)$being an algebra, $\psi:=\Phi-(\Phi \cdot v) v \in H^{1}\left(B_{R}^{+} ; \mathbb{R}^{d},|z|^{a} \mathrm{~d} \mathbf{x}\right) \cap$ $L^{\infty}\left(B_{R}^{+}\right)$. It also satisfies $\psi=0$ on $\partial^{+} B_{R}$, and by construction, we have $v \cdot \psi=0$ a.e. in $B_{R}^{+}$. Now we consider $\xi:=\rho \psi \in H^{1}\left(B_{R}^{+} ; \mathbb{R}^{d},|z|^{a} \mathrm{~d} \mathbf{x}\right) \cap L^{\infty}\left(\mathbb{R}^{n}\right)$, which still satisfies $\xi=0$ on $\partial^{+} B_{R}$, and $u^{\mathrm{e}} \cdot \xi=0$ in $B_{R}^{+}$. In particular, $u \cdot \xi=0$ on $\partial^{0} B_{R}^{+}$. 
By Proposition 3.13, the map $u^{\mathrm{e}}$ is a weighted weakly harmonic map with free boundary in the half ball $B_{R}^{+}$, i.e., it satisfies (3.12). Hence,

$$
\int_{B_{R}^{+}} z^{a} \nabla u^{\mathrm{e}} \cdot \nabla \xi \mathrm{d} \mathbf{x}=0 .
$$

On the other hand, $\partial_{i} u^{\mathrm{e}}=\partial_{i} \rho v+\rho \partial_{i} v$ and $\partial_{i} \xi=\partial_{i} \rho \psi+\rho \partial_{i} \psi$ in $B_{R}^{+}$for $i=1, \ldots, n+1$. Then we notice that $v \cdot \psi=0$ implies $v \cdot \partial_{i} \psi=-\partial_{i} v \cdot \psi$ in $B_{R}^{+}$for $i=1, \ldots, n+1$. In the same way, the fact that $|v|^{2}=1$ leads to $v \cdot \partial_{i} v=0$ in $B_{R}^{+}$for $i=1, \ldots, n+1$. As a consequence,

$$
\partial_{i} u^{\mathrm{e}} \cdot \partial_{i} \xi=\left(\partial_{i} \rho v+\rho \partial_{i} v\right) \cdot\left(\partial_{i} \rho \psi+\rho \partial_{i} \psi\right)=\rho^{2} \partial_{i} v \cdot \partial_{i} \psi \quad \text { a.e. in } B_{R}^{+},
$$

for $i=1, \ldots, n+1$. Inserting this identity in (5.3) yields

$$
\int_{B_{R}^{+}} z^{a} \rho^{2} \nabla v \cdot \nabla \psi \mathrm{d} \mathbf{x}=0 .
$$

To conclude, we notice that

$\partial_{i} v \cdot \partial_{i} \psi=\partial_{i} v \cdot\left(\partial_{i} \Phi-(v \cdot \Phi) \partial_{i} v-\left(\partial_{i} v \cdot \Phi+v \cdot \partial_{i} \Phi\right) v\right)=\partial_{i} v \cdot \partial_{i} \Phi-\left|\partial_{i} v\right|^{2} v \cdot \Phi \quad$ a.e. in $B_{R}^{+}$, for $i=1, \ldots, n+1$. Using this last identity in (5.4) leads to the announced conclusion.

As usual, to deal with homogeneous Neumann condition, we extend the equation to the whole ball by symmetry. In this way, proving estimates up to the boundary reduces to prove interior estimates.

Corollary 5.4. Let $u \in \widehat{H}^{s}\left(D_{2 R} ; \mathbb{S}^{d-1}\right)$ be a weakly s-harmonic map in $D_{2 R}$. Assume that $\left|u^{\mathrm{e}}\right| \geqslant 1 / 2$ a.e. in $B_{R}^{+}$. Then the function $\rho$ and the map $v$ defined by

$$
\rho(\mathbf{x}):= \begin{cases}\left|u^{\mathrm{e}}(x, z)\right| & \text { if } \mathbf{x}=(x, z) \in B_{R}^{+} \\ \left|u^{\mathrm{e}}(x,-z)\right| & \text { if } \mathbf{x}=(x, z) \in B_{R}^{-}\end{cases}
$$

and

$$
v(\mathbf{x}):= \begin{cases}u^{\mathrm{e}}(x, z) / \rho(\mathbf{x}) & \text { if } \mathbf{x}=(x, z) \in B_{R}^{+} \\ u^{\mathrm{e}}(x,-z) / \rho(\mathbf{x}) & \text { if } \mathbf{x}=(x, z) \in B_{R}^{-}\end{cases}
$$

belong to $H^{1}\left(B_{R},|z|^{a} \mathrm{~d} \mathbf{x}\right) \cap L^{\infty}\left(B_{R}\right)$ and $H^{1}\left(B_{R} ; \mathbb{R}^{d},|z|^{a} \mathrm{~d} \mathbf{x}\right) \cap L^{\infty}\left(B_{R}\right)$ respectively, and

$$
\int_{B_{R}}|z|^{a} \rho^{2} \nabla v \cdot \nabla \Phi \mathrm{d} \mathbf{x}=\int_{B_{R}}|z|^{a} \rho^{2}|\nabla v|^{2} v \cdot \Phi \mathrm{d} \mathbf{x}
$$

holds for every $\Phi \in H^{1}\left(B_{R} ; \mathbb{R}^{d},|z|^{a} \mathrm{~d} \mathbf{x}\right) \cap L^{\infty}\left(B_{R}\right)$ such that $\Phi=0$ on $\partial B_{R}$.

Proof. The fact that $\rho$ and $v$ belong to $H^{1}\left(B_{R},|z|^{a} \mathrm{~d} \mathbf{x}\right) \cap L^{\infty}\left(B_{R}\right)$ and $H^{1}\left(B_{R} ; \mathbb{R}^{d},|z|^{a} \mathrm{~d} \mathbf{x}\right) \cap$ $L^{\infty}\left(B_{R}\right)$ respectively follows from Lemma 5.3 together with the symmetry with respect to the hyperplane $\{z=0\}$.

We now consider an arbitrary $\Phi \in H^{1}\left(B_{R} ; \mathbb{R}^{d},|z|^{a} \mathrm{~d} \mathbf{x}\right) \cap L^{\infty}\left(B_{R}\right)$ satisfying $\Phi=0$ on $\partial B_{R}$. We split $\Phi$ into its symmetric and anti-symmetric parts defined by

$$
\Phi^{s}(x, z):=\frac{\Phi(x, z)+\Phi(x,-z)}{2} \text { and } \Phi^{a}(x, z):=\frac{\Phi(x, z)-\Phi(x,-z)}{2} .
$$

Clearly, $\Phi^{s}, \Phi^{a} \in H^{1}\left(B_{R} ; \mathbb{R}^{d},|z|^{a} \mathrm{~d} \mathbf{x}\right) \cap L^{\infty}\left(B_{R}\right)$ and $\Phi^{s}=\Phi^{a}=0$ on $\partial B_{R}$. By construction, we have $\Phi^{s}(x,-z)=\Phi^{s}(x, z)$ and $\Phi^{a}(x,-z)=-\Phi^{a}(x, z)$, so that $\partial_{z} \Phi^{s}(x, z)=-\partial_{z} \Phi^{s}(x,-z)$ and $\partial_{z} \Phi^{a}(x, z)=\partial_{z} \Phi^{a}(x,-z)$. The map $v$ being symmetric with respect to $\{z=0\}$, it also satisfies $\partial_{z} v(x, z)=-\partial_{z} v(x,-z)$. Therefore,

$$
\left(\nabla v \cdot \nabla \Phi^{s}\right)(x, z)=\left(\nabla v \cdot \nabla \Phi^{s}\right)(x,-z) \quad \text { and } \quad\left(\nabla v \cdot \nabla \Phi^{a}\right)(x, z)=-\left(\nabla v \cdot \nabla \Phi^{a}\right)(x,-z) .
$$

As a first consequence,

$$
\int_{B_{R}}|z|^{a} \rho^{2} \nabla v \cdot \nabla \Phi^{a} \mathrm{~d} \mathbf{x}=0
$$

Since $\left(v \cdot \Phi^{a}\right)(x,-z)=-\left(v \cdot \Phi^{a}\right)(x, z)$, we also have

$$
\int_{B_{R}}|z|^{a} \rho^{2}|\nabla v|^{2} v \cdot \Phi^{a} \mathrm{~d} \mathbf{x}=0 .
$$


Then we infer from Lemma 5.3 that

$$
\begin{aligned}
\int_{B_{R}}|z|^{a} \rho^{2} \nabla v \cdot \nabla \Phi^{s} \mathrm{~d} \mathbf{x}=2 & \int_{B_{R}^{+}} z^{a} \rho^{2} \nabla v \cdot \nabla \Phi^{s} \mathrm{~d} \mathbf{x} \\
& =2 \int_{B_{R}^{+}} z^{a} \rho^{2}|\nabla v|^{2} v \cdot \Phi^{s} \mathrm{~d} \mathbf{x}=\int_{B_{R}}|z|^{a} \rho^{2}|\nabla v|^{2} v \cdot \Phi^{s} \mathrm{~d} \mathbf{x} .
\end{aligned}
$$

Gathering (5.8), (5.9), and (5.10) leads to (5.7), and the proof is complete.

Proof of Proposition 5.2. Rescaling variables, we can assume without loss of generality that $R=1$. Throughout the proof, we shall write for a measurable set $A \subseteq \mathbb{R}^{n+1}$,

$$
|A|_{a}:=\int_{A}|z|^{a} \mathrm{~d} \mathbf{x}
$$

and we notice that for $\mathbf{y} \in \mathbb{R}^{n} \times\{0\}$,

$$
\left|B_{r}(\mathbf{y})\right|_{a}=\left|B_{r}\right|_{a}=\left|B_{1}\right|_{a} r^{n+2-2 s} .
$$

We start by applying Corollary 5.4 to consider the (symmetrized) modulus function $\rho$ and the (symmetrized) phase map $v$ defined by (5.5) and (5.6), respectively. Since $u^{\mathrm{e}}$ belongs to $C^{0, \beta}\left(B_{R}^{+}\right)$and $\left|u^{\mathrm{e}}\right| \geqslant 1 / 2$ in $B_{R}^{+}$, it follows that $v \in C^{0, \beta}\left(B_{R}\right)$, and $\rho \in C^{0, \beta}\left(B_{R}\right)$ with $\rho \geqslant 1 / 2$ in $B_{R}$. By Corollary 5.4, $v$ satisfies (5.7), and from this equation we shall obtain that $v \in C^{0,1}\left(B_{R / 3}\right)$. We proceed in several steps.

Step 1. Let us fix $\mathbf{y} \in D_{1 / 2} \times\{0\}$ and $r \in(0,1 / 2]$. We consider the unique weak solution $w \in H^{1}\left(B_{r}(\mathbf{y}) ; \mathbb{R}^{d},|z|^{a} \mathrm{~d} \mathbf{x}\right)$ of

$$
\begin{cases}\operatorname{div}\left(|z|^{a} \nabla w\right)=0 & \text { in } B_{r}(\mathbf{y}), \\ w=v & \text { on } \partial B_{r}(\mathbf{y}),\end{cases}
$$

see Appendix A. The map $v$ being continuous in $\bar{B}_{r}(\mathbf{y})$, it follows from Lemma A.3 that $w \in C^{0}\left(\bar{B}_{r}(\mathbf{y})\right)$. Moreover, since $v$ is symmetric with respect to the hyperplane $\{z=0\}$, Lemma A.2 tells us that $w$ is also symmetric with respect to $\{z=0\}$.

Now we estimate through Minkowski's inequality,

$$
\begin{aligned}
\left(\frac{1}{\left|B_{r / 2}\right|_{a}} \int_{B_{r / 2}(\mathbf{y})}|z|^{a} \rho^{2}|\nabla v|^{2} \mathrm{~d} \mathbf{x}\right)^{1 / 2} & \leqslant\left(\frac{1}{\left|B_{r / 2}\right|_{a}} \int_{B_{r / 2}(\mathbf{y})}|z|^{a} \rho^{2}|\nabla w|^{2} \mathrm{~d} \mathbf{x}\right)^{1 / 2} \\
& +C\left(\frac{1}{\left|B_{r}\right|_{a}} \int_{B_{r}(\mathbf{y})}|z|^{a} \rho^{2}|\nabla(v-w)|^{2} \mathrm{~d} \mathbf{x}\right)^{1 / 2},
\end{aligned}
$$

and our first aim is to estimate the two terms in the right hand side of this inequality.

From the definition of $\eta$ and the fact that $0 \leqslant \rho \leqslant 1$, we have

$$
\left|\rho^{2}(\mathbf{x})-\rho^{2}(\mathbf{y})\right| \leqslant 2 \eta|\mathbf{x}-\mathbf{y}|^{\beta} \leqslant C \eta r^{\beta} \quad \forall \mathbf{x} \in B_{r}(\mathbf{y}) .
$$

Consequently,

$$
\begin{aligned}
\int_{B_{r / 2}(\mathbf{y})}|z|^{a} \rho^{2}|\nabla w|^{2} \mathrm{~d} \mathbf{x} & \leqslant \rho^{2}(\mathbf{y}) \int_{B_{r / 2}(\mathbf{y})}|z|^{a}|\nabla w|^{2} \mathrm{~d} \mathbf{x}+\int_{B_{r / 2}(\mathbf{y})}|z|^{a}\left|\rho^{2}-\rho^{2}(\mathbf{y})\right||\nabla w|^{2} \mathrm{~d} \mathbf{x} \\
& \leqslant\left(1+C \eta r^{\beta}\right) \int_{B_{r / 2}(\mathbf{y})}|z|^{a}|\nabla w|^{2} \mathrm{~d} \mathbf{x}
\end{aligned}
$$

Since $w$ is symmetric with respect to $\{z=0\}$, we infer from Lemma A.4 and (5.11) that the function

$$
t \in(0, r] \mapsto \frac{1}{\left|B_{t}\right|_{a}} \int_{B_{t}(\mathbf{y})}|z|^{a}|\nabla w|^{2} \mathrm{~d} \mathbf{x}
$$


is nondecreasing. Hence,

$$
\begin{aligned}
\frac{1}{\left|B_{r / 2}\right|_{a}} \int_{B_{r / 2}(\mathbf{y})}|z|^{a} \rho^{2}|\nabla w|^{2} \mathrm{~d} \mathbf{x} \leqslant \frac{\left(1+C \eta r^{\beta}\right)}{\left|B_{r}\right|_{a}} \int_{B_{r}(\mathbf{y})}|z|^{a}|\nabla w|^{2} \mathrm{~d} \mathbf{x} & \\
& \leqslant \frac{\left(1+C \eta r^{\beta}\right)}{\left|B_{r}\right|_{a}} \int_{B_{r}(\mathbf{y})}|z|^{a}|\nabla v|^{2} \mathrm{~d} \mathbf{x},
\end{aligned}
$$

where we have used the minimality of $w$ stated in Lemma A.1 in the last inequality. Using $\rho(\mathbf{y})=1$ and $\rho \geqslant 1 / 2$, we now estimate as above,

$$
\begin{aligned}
\int_{B_{r}(\mathbf{y})}|z|^{a}|\nabla v|^{2} \mathrm{~d} \mathbf{x} & \leqslant \int_{B_{r}(\mathbf{y})}|z|^{a} \rho^{2}|\nabla v|^{2} \mathrm{~d} \mathbf{x}+\int_{B_{r}(\mathbf{y})}|z|^{a}\left|\rho^{2}-\rho^{2}(\mathbf{y})\right||\nabla v|^{2} \mathrm{~d} \mathbf{x} \\
& \leqslant\left(1+C \eta r^{\beta}\right) \int_{B_{r}(\mathbf{y})}|z|^{a} \rho^{2}|\nabla v|^{2} \mathrm{~d} \mathbf{x}
\end{aligned}
$$

to reach

$$
\frac{1}{\left|B_{r / 2}\right|_{a}} \int_{B_{r / 2}(\mathbf{y})}|z|^{a} \rho^{2}|\nabla w|^{2} \mathrm{~d} \mathbf{x} \leqslant \frac{\left(1+C \eta r^{\beta}\right)^{2}}{\left|B_{r}\right|_{a}} \int_{B_{r}(\mathbf{y})}|z|^{a} \rho^{2}|\nabla v|^{2} \mathrm{~d} \mathbf{x} .
$$

Next, we recall that $v-w \in H^{1}\left(B_{r}(\mathbf{y}) ; \mathbb{R}^{d},|z|^{a} \mathrm{~d} \mathbf{x}\right)$ satisfies $v-w=0$ on $\partial B_{r}(\mathbf{y})$. Hence, we can apply Corollary 5.4 to deduce that

$$
\begin{gathered}
\int_{B_{r}(\mathbf{y})}|z|^{a} \rho^{2}|\nabla(v-w)|^{2} \mathrm{~d} \mathbf{x}=\int_{B_{r}(\mathbf{y})}|z|^{a} \rho^{2} \nabla v \cdot \nabla(v-w) \mathrm{d} \mathbf{x}-\int_{B_{r}(\mathbf{y})}|z|^{a} \rho^{2} \nabla w \cdot \nabla(v-w) \mathrm{d} \mathbf{x} \\
=\int_{B_{r}(\mathbf{y})}|z|^{a} \rho^{2}|\nabla v|^{2} v \cdot(v-w) \mathrm{d} \mathbf{x}-\int_{B_{r}(\mathbf{y})}|z|^{a} \rho^{2} \nabla w \cdot \nabla(v-w) \mathrm{d} \mathbf{x}
\end{gathered}
$$

On the other hand, the equation (5.12) satisfied by $w$ yields

$$
\begin{aligned}
\int_{B_{r}(\mathbf{y})}|z|^{a} \rho^{2} \nabla w \cdot \nabla(v-w) \mathrm{d} \mathbf{x} & =\rho^{2}(\mathbf{y}) \int_{B_{r}(\mathbf{y})}|z|^{a} \nabla w \cdot \nabla(v-w) \mathrm{d} \mathbf{x} \\
& +\int_{B_{r}(\mathbf{y})}|z|^{a}\left(\rho^{2}-\rho^{2}(\mathbf{y})\right) \nabla w \cdot \nabla(v-w) \mathrm{d} \mathbf{x} \\
& =\int_{B_{r}(\mathbf{y})}|z|^{a}\left(\rho^{2}-\rho^{2}(\mathbf{y})\right) \nabla w \cdot \nabla(v-w) \mathrm{d} \mathbf{x} .
\end{aligned}
$$

By (5.14) and the minimality of $w$, we have

$$
\begin{aligned}
\left.\left|\int_{B_{r}(\mathbf{y})}\right| z\right|^{a}\left(\rho^{2}-\rho^{2}(\mathbf{y})\right) \nabla w \cdot \nabla(v-w) \mathrm{d} \mathbf{x} \mid & \leqslant C \eta r^{\beta} \int_{B_{r}(\mathbf{y})}|z|^{a}|\nabla w||\nabla(v-w)| \mathrm{d} \mathbf{x} \\
& \leqslant C \eta r^{\beta} \int_{B_{r}(\mathbf{y})}|z|^{a}\left(|\nabla w|^{2}+|\nabla v|^{2}\right) \mathrm{d} \mathbf{x} \\
& \leqslant C \eta r^{\beta} \int_{B_{r}(\mathbf{y})}|z|^{a}|\nabla v|^{2} \mathrm{~d} \mathbf{x} \\
& \leqslant C \eta r^{\beta} \int_{B_{r}(\mathbf{y})}|z|^{a} \rho^{2}|\nabla v|^{2} \mathrm{~d} \mathbf{x},
\end{aligned}
$$

where we have used that $\rho \geqslant 1 / 2$ in the last inequality. Combining (5.17), (5.18), (5.19), and using that $|v|=1$, we infer that

$$
\int_{B_{r}(\mathbf{y})}|z|^{a} \rho^{2}|\nabla(v-w)|^{2} \mathrm{~d} \mathbf{x} \leqslant\left(\|v-w\|_{L^{\infty}\left(B_{r}(\mathbf{y})\right)}+C \eta r^{\beta}\right) \int_{B_{r}(\mathbf{y})}|z|^{a} \rho^{2}|\nabla v|^{2} \mathrm{~d} \mathbf{x} .
$$

Let us now bound $\|v-w\|_{L^{\infty}\left(B_{r}(\mathbf{y})\right)}$. First, notice that for $\mathbf{x} \in B_{r}(\mathbf{y})$,

$$
|v(\mathbf{x})-w(\mathbf{x})| \leqslant|v(\mathbf{x})-v(\mathbf{y})|+|w(\mathbf{x})-v(\mathbf{y})| \leqslant C \eta r^{\beta}+|w(\mathbf{x})-v(\mathbf{y})| .
$$

Next we observe that for each $i=1, \ldots, d$, the scalar function $w^{i}-v^{i}(\mathbf{y}) \in H^{1}\left(B_{r}(\mathbf{y}),|z|^{a} \mathrm{~d} \mathbf{x}\right)$ satisfies in the weak sense

$$
\begin{cases}\operatorname{div}\left(|z|^{a} \nabla\left(w^{i}-v^{i}(\mathbf{y})\right)\right)=0 & \text { in } B_{r}(\mathbf{y}) \\ w^{i}-v^{i}(\mathbf{y})=v^{i}-v^{i}(\mathbf{y}) & \text { on } \partial B_{r}(\mathbf{y}) .\end{cases}
$$


It then follows from Lemma A.3 that for each $i=1, \ldots, d$,

$$
\left\|w^{i}-v^{i}(\mathbf{y})\right\|_{L^{\infty}\left(B_{r}(\mathbf{y})\right)} \leqslant\left\|v^{i}-v^{i}(\mathbf{y})\right\|_{L^{\infty}\left(\partial B_{r}(\mathbf{y})\right)} \leqslant\|v-v(\mathbf{y})\|_{L^{\infty}\left(\partial B_{r}(\mathbf{y})\right)} \leqslant C \eta r^{\beta} .
$$

Back to (5.21), we have thus obtained

$$
\|w-v\|_{L^{\infty}\left(B_{r}(\mathbf{y})\right)} \leqslant C \eta r^{\beta} .
$$

Using this estimate in (5.20), we derive that

$$
\int_{B_{r}(\mathbf{y})}|z|^{a} \rho^{2}|\nabla(v-w)|^{2} \mathrm{~d} \mathbf{x} \leqslant C \eta r^{\beta} \int_{B_{r}(\mathbf{y})}|z|^{a} \rho^{2}|\nabla v|^{2} \mathrm{~d} \mathbf{x} .
$$

Now, inserting estimates (5.16) and (5.22) in (5.13), and then squaring both sides of the resulting inequality, we are led to

$$
\frac{1}{\left|B_{r / 2}\right|_{a}} \int_{B_{r / 2}(\mathbf{y})}|z|^{a} \rho^{2}|\nabla v|^{2} \mathrm{~d} \mathbf{x} \leqslant \frac{\left(1+C_{\eta} r^{\beta / 2}\right)}{\left|B_{r}\right|_{a}} \int_{B_{r}(\mathbf{y})}|z|^{a} \rho^{2}|\nabla v|^{2} \mathrm{~d} \mathbf{x},
$$

for a constant $C_{\eta}=C_{\eta}(\eta, n, s)$. Iterating this inequality along dyadic radii $r_{k}:=2^{-k}$ with $k \geqslant 1$, we deduce that

$$
\begin{aligned}
\frac{1}{\mid B_{r_{k+1} \mid a}} \int_{B_{r_{k+1}}(\mathbf{y})}|z|^{a} \rho^{2}|\nabla v|^{2} \mathrm{~d} \mathbf{x} \leqslant\left(\prod_{j=1}^{k}\left(1+C_{\eta} 2^{-j \beta / 2}\right)\right) & \frac{1}{\left|B_{1 / 2}\right|_{a}} \int_{B_{1 / 2}(\mathbf{y})}|z|^{a} \rho^{2}|\nabla v|^{2} \mathrm{~d} \mathbf{x} \\
& \leqslant C_{\eta, \beta} \int_{B_{1}}|z|^{a} \rho^{2}|\nabla v|^{2} \mathrm{~d} \mathbf{x}, \quad
\end{aligned}
$$

for a constant $C_{\eta, \beta}=C_{\eta, \beta}(\eta, \beta, n, s)$. Next, for an arbitrary radius $r \in(0,1 / 2]$, we consider the integer $k \geqslant 1$ satisfying $r_{k+1}<r \leqslant r_{k}$, and estimate

$$
\frac{1}{\left|B_{r}\right|_{a}} \int_{B_{r}(\mathbf{y})}|z|^{a} \rho^{2}|\nabla v|^{2} \mathrm{~d} \mathbf{x} \leqslant \frac{2^{n+2-2 s}}{\left|B_{r_{k}}\right|_{a}} \int_{B_{r_{k}}(\mathbf{y})}|z|^{a} \rho^{2}|\nabla v|^{2} \mathrm{~d} \mathbf{x}
$$

to conclude from (5.23) and the symmetry of $v$ and $\rho$ with respect to $\{z=0\}$ that

$$
\frac{1}{\left|B_{r}\right|_{a}} \int_{B_{r}(\mathbf{y})}|z|^{a} \rho^{2}|\nabla v|^{2} \mathrm{~d} \mathbf{x} \leqslant C_{\eta, \beta} \int_{B_{1}^{+}}|z|^{a} \rho^{2}|\nabla v|^{2} \mathrm{~d} \mathbf{x} \quad \forall r \in(0,1 / 2] .
$$

Noticing that $\left.|\nabla u|^{\mathrm{e}}\right|^{2}=|\nabla \rho|^{2}+\rho^{2}|\nabla v|^{2}$, and in view of the arbitrariness of $\mathbf{y}$, we have thus proved that

$$
\frac{1}{\left|B_{r}\right|_{a}} \int_{B_{r}(\mathbf{y})}|z|^{a} \rho^{2}|\nabla v|^{2} \mathrm{~d} \mathbf{x} \leqslant C_{\eta, \beta} \int_{B_{1}^{+}}|z|^{a}\left|\nabla u^{\mathrm{e}}\right|^{2} \mathrm{~d} \mathbf{x} \quad \forall \mathbf{y} \in D_{1 / 2} \times\{0\}, \forall r \in(0,1 / 2] .
$$

Step 2. Our main goal in this step is to obtain an estimate similar to (5.24) for balls which are not centered at points of $\{z=0\}$. By symmetry of $v$ and $\rho$ with respect to $\{z=0\}$, it is enough to consider balls centered at points of $\mathbb{R}_{+}^{n+1}$.

Let us fix an arbitrary point $\mathbf{y}=(y, t) \in B_{1 / 3}^{+}$, and notice that $\bar{B}_{t / 2}(\mathbf{y}) \subseteq B_{1}^{+}$. We also consider an arbitrary radius $r \in(0, t / 2]$ (so that $\bar{B}_{r}(\mathbf{y}) \subseteq B_{1}^{+}$). As in Step 1, we introduce the (weak) solution $w \in H^{1}\left(B_{r}(\mathbf{y}) ; \mathbb{R}^{d},|z|^{a} \mathrm{~d} \mathbf{x}\right)$ of (5.12). Exactly as in (5.13), we have

$$
\begin{aligned}
\left(\left(\frac{2}{r}\right)^{n+1} \int_{B_{r / 2}(\mathbf{y})}|z|^{a} \rho^{2}|\nabla v|^{2} \mathrm{~d} \mathbf{x}\right)^{1 / 2} & \leqslant\left(\left(\frac{2}{r}\right)^{n+1} \int_{B_{r / 2}(\mathbf{y})}|z|^{a} \rho^{2}|\nabla w|^{2} \mathrm{~d} \mathbf{x}\right)^{1 / 2} \\
& +C\left(\frac{1}{r^{n+1}} \int_{B_{r}(\mathbf{y})}|z|^{a} \rho^{2}|\nabla(v-w)|^{2} \mathrm{~d} \mathbf{x}\right)^{1 / 2} .
\end{aligned}
$$

Arguing precisely as in Step 1, we derive that (5.22) still holds. Then, we estimate as in (5.15),

$$
\int_{B_{r / 2}(\mathbf{y})}|z|^{a} \rho^{2}|\nabla w|^{2} \mathrm{~d} \mathbf{x} \leqslant\left(\rho^{2}(\mathbf{y})+C \eta r^{\beta}\right) \int_{B_{r / 2}(\mathbf{y})}|z|^{a}|\nabla w|^{2} \mathrm{~d} \mathbf{x} .
$$


Applying Lemma A.5 with $\theta=t / r$ and then the minimality of $w$, we obtain

$$
\begin{aligned}
\left(\frac{2}{r}\right)^{n+1} \int_{B_{r / 2}(\mathbf{y})}|z|^{a}|\nabla w|^{2} \mathrm{~d} \mathbf{x} \leqslant\left(1+\frac{C r}{t}\right) \frac{1}{r^{n+1}} \int_{B_{r}(\mathbf{y})}|z|^{a}|\nabla w|^{2} \mathrm{~d} \mathbf{x} & \\
& \leqslant\left(1+\frac{C r}{t}\right) \frac{1}{r^{n+1}} \int_{B_{r}(\mathbf{y})}|z|^{a}|\nabla v|^{2} \mathrm{~d} \mathbf{x} .
\end{aligned}
$$

Combining (5.26) with (5.27), and using again the Hölder continuity of $\rho^{2}$ (as in (5.14)) together with $1 / 2 \leqslant \rho \leqslant 1$, we deduce that

$$
\left(\frac{2}{r}\right)^{n+1} \int_{B_{r / 2}(\mathbf{y})}|z|^{a} \rho^{2}|\nabla w|^{2} \mathrm{~d} \mathbf{x} \leqslant\left(1+C\left(\eta r^{\beta}+r / t\right)\right) \frac{1}{r^{n+1}} \int_{B_{r}(\mathbf{y})}|z|^{a} \rho^{2}|\nabla v|^{2} \mathrm{~d} \mathbf{x} .
$$

Inserting (5.22) and (5.28) in (5.25), we infer that

$$
\frac{1}{\left|B_{r / 2}(\mathbf{y})\right|} \int_{B_{r / 2}(\mathbf{y})}|z|^{a} \rho^{2}|\nabla v|^{2} \mathrm{~d} \mathbf{x} \leqslant \frac{1+C_{\eta}\left(r^{\beta / 2}+r / t\right)}{\left|B_{r}(\mathbf{y})\right|} \int_{B_{r}(\mathbf{y})}|z|^{a} \rho^{2}|\nabla v|^{2} \mathrm{~d} \mathbf{x},
$$

for a constant $C_{\eta}=C_{\eta}(\eta, n, s)$. Arguing as Step 1 (using the dyadic radii $r_{k}:=2^{-k} t$ ), the arbitrariness of $r \in(0, t / 2]$ in this latter estimate implies that

$$
\frac{1}{\left|B_{r}(\mathbf{y})\right|} \int_{B_{r}(\mathbf{y})}|z|^{a} \rho^{2}|\nabla v|^{2} \mathrm{~d} \mathbf{x} \leqslant \frac{C_{\eta, \beta}}{\left|B_{t / 2}(\mathbf{y})\right|} \int_{B_{t / 2}(\mathbf{y})}|z|^{a} \rho^{2}|\nabla v|^{2} \mathrm{~d} \mathbf{x} \quad \forall r \in(0, t / 2],
$$

for a constant $C_{\eta, \beta}=C_{\eta, \beta}(\eta, \beta, n, s)$. Then, we notice that for every radius $r \in(0, t / 2]$,

$$
\left|B_{r}(\mathbf{y})\right|_{a} \leqslant \begin{cases}t^{a}(1+r / t)^{a}\left|B_{r}(\mathbf{y})\right| & \text { if } s \leqslant 1 / 2 \\ t^{a}(1-r / t)^{a}\left|B_{r}(\mathbf{y})\right| & \text { if } s>1 / 2\end{cases}
$$

and

$$
\left|B_{r}(\mathbf{y})\right|_{a} \geqslant \begin{cases}t^{a}(1-r / t)^{a}\left|B_{r}(\mathbf{y})\right| & \text { if } s \leqslant 1 / 2, \\ t^{a}(1+r / t)^{a}\left|B_{r}(\mathbf{y})\right| & \text { if } s>1 / 2 .\end{cases}
$$

Consequently, dividing (5.29) by $t^{a}$, we obtain

$$
\frac{1}{\left|B_{r}(\mathbf{y})\right|_{a}} \int_{B_{r}(\mathbf{y})}|z|^{a} \rho^{2}|\nabla v|^{2} \mathrm{~d} \mathbf{x} \leqslant \frac{C_{\eta, \beta}}{\left|B_{t / 2}(\mathbf{y})\right|_{a}} \int_{B_{t / 2}(\mathbf{y})}|z|^{a} \rho^{2}|\nabla v|^{2} \mathrm{~d} \mathbf{x} \quad \forall r \in(0, t / 2] .
$$

Setting $\widetilde{\mathbf{y}}:=(y, 0) \in D_{1 / 3} \times\{0\}$, we now observe that $B_{t / 2}(\mathbf{y}) \subseteq B_{3 t / 2}^{+}(\widetilde{\mathbf{y}})$ and $3 t / 2 \leqslant 1 / 2$. Using the symmetry of $v$ and $\rho$ with respect to $\{z=0\}$ and (5.24), we deduce that

$$
\begin{aligned}
\frac{1}{\left|B_{t / 2}(\mathbf{y})\right|_{a}} \int_{B_{t / 2}(\mathbf{y})}|z|^{a} \rho^{2}|\nabla v|^{2} \mathrm{~d} \mathbf{x} & \leqslant \frac{C}{\left|B_{3 t / 2}^{+}(\widetilde{\mathbf{y}})\right|_{a}} \int_{B_{3 t / 2}^{+}(\widetilde{\mathbf{y}})}|z|^{a} \rho^{2}|\nabla v|^{2} \mathrm{~d} \mathbf{x} \\
& \leqslant \frac{C}{\left|B_{3 t / 2}(\widetilde{\mathbf{y}})\right|_{a}} \int_{B_{3 t / 2}(\widetilde{\mathbf{y}})}|z|^{a} \rho^{2}|\nabla v|^{2} \mathrm{~d} \mathbf{x} \\
& \leqslant C_{\eta, \beta} \int_{B_{1}^{+}}|z|^{a}\left|\nabla u^{\mathrm{e}}\right|^{2} \mathrm{~d} \mathbf{x} .
\end{aligned}
$$

Combining (5.30) and (5.31), and in view of the arbitrariness of $\mathbf{y}$, we infer that

$$
\frac{1}{\left|B_{r}(\mathbf{y})\right|_{a}} \int_{B_{r}(\mathbf{y})}|z|^{a} \rho^{2}|\nabla v|^{2} \mathrm{~d} \mathbf{x} \leqslant C_{\eta, \beta} \int_{B_{1}^{+}}|z|^{a}\left|\nabla u^{\mathrm{e}}\right|^{2} \mathrm{~d} \mathbf{x} \quad \forall \mathbf{y}=(y, t) \in B_{1 / 3}^{+}, \forall r \in(0, t / 2] .
$$

Still by symmetry of $v$ and $\rho$, this estimate actually holds for every $\mathbf{y}=(y, t) \in B_{1 / 3} \backslash\{z=0\}$ and $r \in(0,|t| / 2)$. By Lebesgue's differentiation theorem, we have thus proved that

$$
\rho^{2}|\nabla v|^{2} \leqslant C_{\eta, \beta} \int_{B_{1}^{+}}|z|^{a}\left|\nabla u^{\mathrm{e}}\right|^{2} \mathrm{~d} \mathbf{x} \quad \text { a.e. in } B_{1 / 3},
$$

and the conclusion follows from the fact that $\rho \geqslant 1 / 2$.

Proof of Theorem 5.1. Once again, rescaling variables, we can assume that $R=1$. Under condition (5.1), Corollary 4.2 says that $u^{\mathrm{e}} \in C^{0, \beta_{1}}\left(B_{\kappa_{1}}^{+}\right)$and $\left[u^{\mathrm{e}}\right]_{C^{0, \beta_{1}}\left(B_{\kappa_{1}}^{+}\right)}$is bounded by a constant depending only on $n$ and $s$. Since $\left|u^{\mathrm{e}}\right|=|u|=1$ on $\partial^{0} B_{\kappa_{1}}^{+}$, we can thus find a constant $\boldsymbol{\kappa}_{2}=\boldsymbol{\kappa}_{2}(n, s) \in(0,1)$ such that $6 \boldsymbol{\kappa}_{2} \leqslant \boldsymbol{\kappa}_{1}$ and $\left|u^{\mathrm{e}}\right| \geqslant 1 / 2$ in $B_{3 \kappa_{2}}^{+}$. Since $\beta_{1}=\beta_{1}(n, s)$, and 
$\left(3 \kappa_{2}\right)^{\beta_{1}}\left[u^{\mathrm{e}}\right]_{C^{0, \beta_{1}}\left(B_{3 \kappa_{2}}^{+}\right)}$is bounded by a constant depending only on $n$ and $s$, Proposition 5.2 implies that $v:=u^{\mathrm{e}} /\left|u^{\mathrm{e}}\right|$ is Lipschitz continuous in $\bar{B}_{\boldsymbol{\kappa}_{2}}^{+}$with

$$
|v(\mathbf{x})-v(\mathbf{y})|^{2} \leqslant C \boldsymbol{\Theta}_{s}\left(u^{\mathrm{e}}, 0, \boldsymbol{\kappa}_{2}\right)|\mathbf{x}-\mathbf{y}|^{2} \leqslant C \boldsymbol{\Theta}_{s}\left(u^{\mathrm{e}}, 0,1\right)|\mathbf{x}-\mathbf{y}|^{2} \quad \forall \mathbf{x}, \mathbf{y} \in \bar{B}_{\boldsymbol{\kappa}_{2}}^{+},
$$

for a constant $C=C(n, s)$. Since $v(\mathbf{x})=u(x)$ for every $\mathbf{x}=(x, 0) \in \partial^{0} B_{\boldsymbol{\kappa}_{2}}^{+}$, the conclusion follows.

\section{HighER ORDER REGULARITY}

We have now reached the final stage of our small energy regularity result where it only remains to prove that a Lipschitz continuous $s$-harmonic map is of class $C^{\infty}$. To achieve this result, we shall apply (local) Schauder type estimates for $(-\Delta)^{s}$. We only refer to [41] for those estimates as it is best suited to our presentation (see also [52]).

Theorem 6.1. Let $u \in \widehat{H}^{s}\left(D_{1} ; \mathbb{S}^{d-1}\right)$ be a weakly s-harmonic map in $D_{1}$. If $u$ is Lipschitz continuous in $D_{1}$, then $u \in C^{\infty}\left(D_{1 / 2}\right)$.

Proof. The proof of Theorem 6.1 follows from a bootstrap procedure. The initiation of the induction consists in passing from Lipschitz regularity to $C^{1, \alpha}$-regularity, and it is the object of Proposition 6.2 in the following subsection. Then we shall prove in Proposition 6.6 that $C^{k, \alpha_{-}}$ regularity upgrades to $C^{k+1, \alpha}$-regularity for every integer $k \geqslant 1$. In applying this bootstrap argument, we first fix an arbitrary point $x_{0} \in D_{1 / 2}$ and an integer $k \geqslant 1$. We translate variables by $x_{0}$ and rescale suitably in order to apply Proposition 6.2 and Proposition 6.6, and then conclude that $u$ is $C^{k, \alpha}$ in a neighborhood of $x_{0}$.

\subsection{Hölder continuity of first order derivatives.}

Proposition 6.2. Let $u \in \widehat{H}^{s}\left(D_{3} ; \mathbb{S}^{d-1}\right)$ be a weakly s-harmonic map in $D_{3}$. If $u$ is Lipschitz continuous in $D_{3}$, then $u \in C^{1, \alpha}\left(D_{r_{*}}\right)$ for every $\alpha \in(0,1)$ and some $r_{*}=r_{*}(n, s) \in(0,1 / 2)$.

One of the main ingredients to obtain an improved regularity is the following elementary lemma.

Lemma 6.3. Let $f: D_{3} \rightarrow \mathbb{R}^{d}$ be a Lipschitz continuous function, $g: D_{3} \rightarrow \mathbb{R}^{d}$ an Hölder continuous function, and $\zeta: D_{1} \rightarrow[0,1]$ a measurable function. Assume that one of the following items holds:

(i) $s \in(0,1 / 2)$ and $g \in C^{0, \alpha}\left(D_{3}\right)$ for some $\alpha \in(2 s, 1]$;

(ii) $s \in(0,1 / 2)$ and $g \in C^{0, \alpha}\left(D_{3}\right)$ for every $\alpha \in(0,2 s)$;

(iii) $s \in[1 / 2,1)$ and $g \in C^{0, \alpha}\left(D_{3}\right)$ for every $\alpha \in(0,1)$.

Then the function

$$
G: x \in D_{1} \mapsto \int_{D_{1}} \frac{(f(x+y)-f(x)) \cdot(g(x+y)-g(x))}{|y|^{n+2 s}} \zeta(y) \mathrm{d} y
$$

belongs to

(1) $C^{0, \alpha}\left(D_{1}\right)$ in case (i);

(2) $C^{0, \alpha^{\prime}}\left(D_{1}\right)$ for every $\alpha^{\prime} \in(0,2 s)$ in case (ii);

(3) $C^{0, \alpha^{\prime}}\left(D_{1}\right)$ for every $\alpha^{\prime} \in(0,2-2 s)$ in case (iii).

Proof. Step 1. We first claim that $G$ is well defined in all cases. To simplify the notation, we write

$$
\Gamma(x, y):=(f(x+y)-f(x)) \cdot(g(x+y)-g(x)) .
$$

Observe that in all cases, we have $1+\alpha>2 s$ (it holds for every $\alpha \in(0,2 s)$ in case (ii), and we can choose such $\alpha \in(0,1)$ in case (iii)). Since $|\Gamma(x, y)| \leqslant C_{f, g, \alpha}|y|^{1+\alpha}$, we have

$$
\int_{D_{1}} \frac{|\Gamma(x, y)|}{|y|^{n+2 s}} \mathrm{~d} y \leqslant C_{f, g, \alpha} \int_{D_{1}} \frac{\mathrm{d} y}{|y|^{n+2 s-(1+\alpha)}} \leqslant C_{f, g, \alpha} \quad \forall x \in D_{1},
$$

for a constant $C_{f, g, \alpha}$ depending only on $f, g, \alpha, n$, and $s$.

Step 2, case (i). Fix arbitrary points $x, h \in D_{1}$. Since

$$
|\Gamma(x+h, y)-\Gamma(x, y)| \leqslant C_{f, g, \alpha}|h|^{\alpha}|y|^{\alpha} \quad \forall y \in D_{1},
$$


we have

$$
|G(x+h)-G(x)| \leqslant C_{f, g, \alpha}|h|^{\alpha} \int_{D_{1}} \frac{1}{|y|^{n+2 s-\alpha}} \mathrm{d} y \leqslant C_{f, g, \alpha}|h|^{\alpha},
$$

for a constant $C_{f, g, \alpha}$ depending only on $f, g, \alpha, n$, and $s$.

Step 3, case (ii). Let us fix an arbitrary $\varepsilon \in(0, s)$. We set $\alpha:=2 s-\varepsilon$ and $\beta:=1-2 \varepsilon$. Since

$$
|\Gamma(x+h, y)-\Gamma(x, y)| \leqslant|\Gamma(x+h, y)|+|\Gamma(x, y)| \leqslant C_{f, g, \varepsilon}|y|^{1+\alpha},
$$

we can use (6.3) to obtain

$$
|\Gamma(x+h, y)-\Gamma(x, y)| \leqslant C_{f, g, \varepsilon}|y|^{(1+\alpha)(1-\beta)}|h|^{\alpha \beta}|y|^{\alpha \beta}=C_{f, g, \varepsilon}|y|^{2 s+\varepsilon}|h|^{\alpha \beta} \quad \forall y \in D_{1} .
$$

Hence,

$$
|G(x+h)-G(x)| \leqslant C_{f, g, \varepsilon}|h|^{\alpha \beta} \int_{D_{1}} \frac{1}{|y|^{n-\varepsilon}} \mathrm{d} y \leqslant C_{f, g, \varepsilon}|h|^{\alpha \beta},
$$

for a constant $C_{f, g, \varepsilon}>0$ depending only on $f, g, \varepsilon, n$, and $s$.

Step 4, case (iii). Now we fix an arbitrary $\varepsilon \in(0,1-s)$, and we set $\alpha:=1-\varepsilon$ and $\beta:=2-2 s-2 \varepsilon$. Then (6.4) still holds, and consequently also (6.5).

Proof of Proposition 6.2. Step 1. We start by fixing a radial cut-off function $\zeta \in \mathscr{D}\left(\mathbb{R}^{n}\right)$ such that $0 \leqslant \zeta \leqslant 1, \zeta=1$ in $D_{1 / 2}$, and $\zeta=0$ in $\mathbb{R}^{n} \backslash D_{3 / 4}$. With $\zeta$ in hands, we rewrite for $x \in D_{1}$,

$$
\begin{aligned}
\int_{\mathbb{R}^{n}} \frac{|u(x)-u(y)|^{2}}{|x-y|^{n+2 s}} \mathrm{~d} y=\int_{\mathbb{R}^{n}} \frac{|u(x+y)-u(x)|^{2}}{|y|^{n+2 s}} \mathrm{~d} y \\
=\int_{D_{1}} \frac{|u(x+y)-u(x)|^{2}}{|y|^{n+2 s}} \zeta(y) \mathrm{d} y+\int_{D_{1 / 2}^{c}} \frac{|u(x+y)-u(x)|^{2}}{|y|^{n+2 s}}(1-\zeta(y)) \mathrm{d} y,
\end{aligned}
$$

and we set

$$
G_{u}(x):=\int_{D_{1}} \frac{|u(x+y)-u(x)|^{2}}{|y|^{n+2 s}} \zeta(y) \mathrm{d} y .
$$

By Lemma 6.3 (applied to $f=g=u$ ), the function $G_{u}$ is Lipschitz continuous in $D_{1}$ for $s \in(0,1 / 2)$, and it belongs to $C^{0, \alpha}\left(D_{1}\right)$ for every $\alpha \in(0,2-2 s)$ for $s \in[1 / 2,1)$.

Concerning the second term in the right hand side of (6.6), we use the identity $|u|^{2}=1$ to rewrite it as

$$
\begin{aligned}
\int_{D_{1 / 2}^{c}} \frac{|u(x+y)-u(x)|^{2}}{|y|^{n+2 s}}(1-\zeta(y)) \mathrm{d} y=\int_{\mathbb{R}^{n}} \frac{2(1-\zeta(y))}{|y|^{n+2 s}} \mathrm{~d} y \\
-\left(\int_{\mathbb{R}^{n}} \frac{2(1-\zeta(y))}{|y|^{n+2 s}} u(x+y) \mathrm{d} y\right) \cdot u(x) .
\end{aligned}
$$

In view of (6.8), it is convenient to introduce the constant $L_{\zeta}>0$ and the function $Z \in C^{\infty}\left(\mathbb{R}^{n}\right)$ given by

$$
L_{\zeta}:=\int_{\mathbb{R}^{n}} \frac{2(1-\zeta(y))}{|y|^{n+2 s}} \mathrm{~d} y \quad \text { and } \quad Z(x):=\frac{2}{L_{\zeta}} \frac{(1-\zeta(x))}{|x|^{n+2 s}} .
$$

In this way, the right-hand side of (6.8) can be written as

$$
H_{u}(x):=L_{\zeta}(1-Z * u(x) \cdot u(x)) \quad \text { for } x \in D_{1} .
$$

Notice that $Z * u \in C^{\infty}\left(\mathbb{R}^{n}\right)$, so that $H_{u}$ is Lipschitz continuous in $D_{1}$.

Summarizing our manipulations in (6.6) and (6.8), we have obtained

$$
\int_{\mathbb{R}^{n}} \frac{|u(x)-u(y)|^{2}}{|x-y|^{n+2 s}} \mathrm{~d} y=G_{u}(x)+H_{u}(x) \quad \forall x \in D_{1} .
$$

Now we introduce the map $F_{u}: D_{1} \rightarrow \mathbb{R}^{d}$ given by

$$
F_{u}(x):=\frac{\gamma_{n, s}}{2}\left(G_{u}(x)+H_{u}(x)\right) u(x) .
$$

Then $F_{u} \in C^{0,1}\left(D_{1}\right)$ for $s \in(0,1 / 2)$, and $F_{u} \in C^{0, \alpha}\left(D_{1}\right)$ for every $\alpha \in(0,2-2 s)$ for $s \in[1 / 2,1)$. 
Step 2. We consider the map $u_{0}: \mathbb{R}^{n} \rightarrow \mathbb{R}^{d}$ given by $u_{0}:=\zeta u$. Then $u_{0} \in C^{0,1}\left(\mathbb{R}^{n}\right)$ and $u_{0}=0$ in $\mathbb{R}^{n} \backslash D_{1}$. In particular, $u_{0} \in H_{00}^{s}\left(D_{1} ; \mathbb{R}^{d}\right)$. A lengthy but straightforward computation shows that

$(-\Delta)^{s} u_{0}=\zeta(-\Delta)^{s} u+\left((-\Delta)^{s} \zeta\right) u-\gamma_{n, s} \int_{\mathbb{R}^{n}} \frac{(\zeta(x)-\zeta(y))(u(x)-u(y))}{|x-y|^{n+2 s}} \mathrm{~d} y \quad$ in $H^{-s}\left(D_{1} ; \mathbb{R}^{d}\right)$,

i.e., in the sense of (2.3). Since $u$ is a weakly $s$-harmonic map in $D_{3}$, it satisfies equation (3.2). In view of Step 1 , we thus have

$$
(-\Delta)^{s} u_{0}=\zeta F_{u}+\left((-\Delta)^{s} \zeta\right) u-\gamma_{n, s} \int_{\mathbb{R}^{n}} \frac{(\zeta(x)-\zeta(y))(u(x)-u(y))}{|x-y|^{n+2 s}} \mathrm{~d} y \quad \text { in } H^{-s}\left(D_{1} ; \mathbb{R}^{d}\right) .
$$

The function $(-\Delta)^{s} \zeta$ being smooth over $\mathbb{R}^{n}$, we infer from Step 1 that $\zeta F_{u}+\left((-\Delta)^{s} \zeta\right) u$ belongs to $C^{0,1}\left(D_{1}\right)$ for $s \in(0,1 / 2)$, and to $C^{0, \alpha}\left(D_{1}\right)$ for every $\alpha \in(0,2-2 s)$ for $s \in[1 / 2,1)$. We now determine the regularity of the last term in the right-hand side of (6.11) arguing as in Step 1. We write it as

$$
\int_{\mathbb{R}^{n}} \frac{(\zeta(x)-\zeta(y))(u(x)-u(y))}{|x-y|^{n+2 s}} \mathrm{~d} y=: I(x)+I I(x),
$$

with

$$
I(x):=\int_{D_{1}} \frac{(\zeta(x+y)-\zeta(x))(u(x+y)-u(x))}{|y|^{n+2 s}} \zeta(y) \mathrm{d} y
$$

and

$$
\begin{aligned}
I I(x) & :=\int_{\mathbb{R}^{n}} \frac{(\zeta(x+y)-\zeta(x))(u(x+y)-u(x))}{|y|^{n+2 s}}(1-\zeta(y)) \mathrm{d} y \\
& =\int_{\mathbb{R}^{n}} \frac{(\zeta(x)-\zeta(y))(u(x)-u(y))}{|x-y|^{n+2 s}}(1-\zeta(x-y)) \mathrm{d} y .
\end{aligned}
$$

By Lemma 6.3, the term $I$ belongs to $C^{0,1}\left(D_{1}\right)$ for $s \in(0,1 / 2)$, and to $C^{0, \alpha}\left(D_{1}\right)$ for every $\alpha \in(0,2-2 s)$ for $s \in[1 / 2,1)$. On the other hand, the function $\zeta$ being smooth and equal to 1 in $D_{1 / 2}$, the term $I I$ has clearly the regularity of $u$ in $D_{1}$, that is $C^{0,1}\left(D_{1}\right)$. Summarizing these considerations, we have shown that $u_{0} \in H^{s}\left(\mathbb{R}^{n} ; \mathbb{R}^{d}\right) \cap L^{\infty}\left(\mathbb{R}^{n}\right)$ is a weak solution of

$$
\begin{cases}(-\Delta)^{s} u_{0}=F_{0} & \text { in } D_{1} \\ u_{0}=0 & \text { in } \mathbb{R}^{n} \backslash D_{1}\end{cases}
$$

for a right-hand side $F_{0}$ which belongs to $C^{0,1}\left(D_{1}\right)$ for $s \in(0,1 / 2)$, and to $C^{0, \alpha}\left(D_{1}\right)$ for every $\alpha \in(0,2-2 s)$ for $s \in[1 / 2,1)$. From well-known (by now) regularity estimates for this equation (see e.g. [41, Section 2]), the map $u_{0}$ belongs to $C^{1, \alpha}\left(D_{1 / 2}\right)$ for every $\alpha \in(0,2 s)$ for $s \in(0,1 / 2)$, and to $C^{1, \alpha}\left(D_{1 / 2}\right)$ for every $\alpha \in(0,1)$ for $s \in[1 / 2,1)$. Since $u_{0}=u$ in $D_{1 / 2}$, the proposition is proved in the case $s \in[1 / 2,1)$, and we obtained $u \in C^{1, \alpha}\left(D_{1 / 2}\right)$ for every $\alpha \in(0,2 s)$ for $s \in(0,1 / 2)$.

Step 3. We now assume that $s \in(0,1 / 2)$, and it remains to prove that $u$ actually belongs to $C^{1, \alpha}\left(D_{r_{*}}\right)$ for every $\alpha \in(0,1)$ and a radius $r_{*} \in(0,1 / 2)$ depending only on $s$. To this purpose, we rescale $u$ by setting $\widetilde{u}(x):=u(x / 6)$, and from Step 2 , we infer that $\widetilde{u} \in C^{1, \alpha}\left(D_{3}\right)$ for every $\alpha \in(0,2 s)$. We shall now make use of the following lemma.

Lemma 6.4. Assume that $s \in(0,1 / 2)$. Let $f: D_{3} \rightarrow \mathbb{R}^{d}$ and $g: D_{3} \rightarrow \mathbb{R}^{d}$ be two $C^{1}$-functions, and $\zeta: D_{1} \rightarrow[0,1]$ a measurable function. Assume that one of the following items holds:

(i) $f, g \in C^{1, \alpha}\left(D_{3}\right)$ for every $\alpha \in(0,2 s)$;

(ii) $f, g \in C^{1, \alpha}\left(D_{3}\right)$ for some $\alpha \in(2 s, 1)$;

Then the function $G: D_{1} \rightarrow \mathbb{R}$ given by (6.1) belongs to

(1) $C^{1, \alpha^{\prime}}\left(D_{1}\right)$ for every $\alpha^{\prime} \in(0,2 s)$ in case (i);

(2) $C^{1, \alpha}\left(D_{1}\right)$ in case (ii); 
and for $x \in D_{1}$,

$$
\begin{aligned}
\partial_{i} G(x)=\int_{D_{1}} \frac{\left(\partial_{i} f(x+y)-\partial_{i} f(x)\right) \cdot(g(x+y)-g(x))}{|y|^{n+2 s}} \zeta(y) \mathrm{d} y \\
+\int_{D_{1}} \frac{(f(x+y)-f(x)) \cdot\left(\partial_{i} g(x+y)-\partial_{i} g(x)\right)}{|y|^{n+2 s}} \zeta(y) \mathrm{d} y,
\end{aligned}
$$

for $i=1, \ldots, n$.

Proof. We keep using notation (6.2). First we fix an arbitrary point $x \in D_{1}$ and we claim that $G$ admits a partial derivative $\partial_{i} G$ at $x$. Indeed, for $t>0$ small enough, we have

$$
\left|\Gamma\left(x+t e_{i}, y\right)-\Gamma(x, y)\right| \leqslant C_{f, g}|y| t \quad \forall y \in D_{1},
$$

since $f$ and $g$ are $C^{1}$ over $D_{3}$. Hence,

$$
\frac{\left|\Gamma\left(x+t e_{i}, y\right)-\Gamma(x, y)\right|}{|y|^{n+2 s} t} \leqslant C_{f, g}|y|^{1-2 s-n} \in L^{1}\left(D_{1}\right),
$$

and it follows from the dominated convergence theorem that $G$ admits a partial derivative $\partial_{i} G$ at $x$ given by formula (6.12).

Next we apply Lemma 6.3 to the right-hand side of (6.12) to deduce that $\partial_{i} G$ is Hölder continuous, and the conclusion follows.

Proof of Proposition 6.2 completed. We consider the function $G_{\widetilde{u}}: D_{1} \rightarrow \mathbb{R}$ as defined in (6.7) with $\tilde{u}$ in place of $u$. By Lemma 6.4 (applied to $f=g=\widetilde{u}$ ), $G_{\widetilde{u}} \in C^{1, \alpha}\left(D_{1}\right)$ for every $\alpha \in(0,2 s)$. On the other hand, the function $H_{\widetilde{u}}: D_{1} \rightarrow \mathbb{R}$ as defined in (6.9) clearly belongs to $C^{1, \alpha}\left(D_{1}\right)$ for every $\alpha \in(0,2 s)$. Consequently, the map $F_{\widetilde{u}}: D_{1} \rightarrow \mathbb{R}^{d}$ as defined in (6.10) also belongs to $C^{1, \alpha}\left(D_{1}\right)$ for every $\alpha \in(0,2 s)$. Since $\widetilde{u}$ is a rescaling of $u$, it is also $s$-harmonic in $D_{1}$, and thus $(-\Delta)^{s} \widetilde{u}=F_{\widetilde{u}}$ in $\mathscr{D}^{\prime}\left(D_{1}\right)$. Next, we keep arguing as in Step 2, and we consider the bounded map $\widetilde{u}_{0}:=\zeta \widetilde{u}$. Applying Lemma 6.4 again, we argue as in Step 2 to infer that $(-\Delta)^{s} \widetilde{u}_{0}=\widetilde{F}_{0}$ in $H^{-s}\left(D_{1} ; \mathbb{R}^{d}\right)$, for a right-hand side $\widetilde{F}_{0} \in C^{1, \alpha}\left(D_{1}\right)$ for every $\alpha \in(0,2 s)$. By the results in [41], we have $\widetilde{u}_{0} \in C^{1, \alpha}\left(D_{1 / 2}\right)$ for every $\alpha \in(0,4 s)$ if $4 s<1$, and $\widetilde{u}_{0} \in C^{1, \alpha}\left(D_{1 / 2}\right)$ for every $\alpha \in(0,1)$ if $4 s \geqslant 1$. Once again, since $\widetilde{u}_{0}=\widetilde{u}$ in $D_{1 / 2}$, we have $\widetilde{u} \in C^{1, \alpha}\left(D_{1 / 2}\right)$ for every $\alpha \in(0,4 s)$ if $4 s<1$, and $\widetilde{u} \in C^{1, \alpha}\left(D_{1 / 2}\right)$ for every $\alpha \in(0,1)$ if $4 s \geqslant 1$.

In the case $s \in[1 / 4,1 / 2)$, we have thus proved that $u \in C^{1, \alpha}\left(D_{1 / 12}\right)$ for every $\alpha \in(0,1)$. Hence it remains to consider the case $s<1 / 4$. In that case, we repeat the preceding argument considering the rescaling $\widehat{u}(x):=\widetilde{u}(x / 6)$. Following the same notation as above, Lemma 6.4 tells us that $G_{\widehat{u}}$ belongs to $C^{1, \alpha}\left(D_{1}\right)$ for every $\alpha \in(0,4 s)$, and hence also $F_{\widehat{u}}$. Then, applying the results of [41] to $\widehat{u}_{0}$, we conclude that $\widehat{u} \in C^{1, \alpha}\left(D_{1 / 2}\right)$ for every $\alpha \in(0,6 s)$ if $6 s<1$, and $\widehat{u} \in C^{1, \alpha}\left(D_{1 / 12}\right)$ for every $\alpha \in(0,1)$ if $6 s \geqslant 1$. Therefore, if $s \geqslant 1 / 6$, then $u \in C^{1, \alpha}\left(D_{1 / 72}\right)$ for every $\alpha \in(0,1)$, which is the announced regularity. On the other hand, if $s \in(0,1 / 6)$, then we repeat the argument. It is now clear that repeating a finite number $\ell$ of times this argument, one reaches the conclusion that $u \in C^{1, \alpha}\left(D_{(6)^{-\ell} / 2}\right)$ for every $\alpha \in(0,1)$, and $\ell$ is essentially the integer part of $1 / 2 s$.

Before closing this subsection, we provide an analogue of Lemma 6.4 in the case $s \in[1 / 2,1)$.

Lemma 6.5. Assume that $s \in[1 / 2,1)$. Let $f: D_{3} \rightarrow \mathbb{R}^{d}$ and $g: D_{3} \rightarrow \mathbb{R}^{d}$ be two $C^{1}$-functions, and $\zeta: D_{1} \rightarrow[0,1]$ a measurable function. If $f$ and $g$ belongs to $C^{1, \alpha}\left(D_{3}\right)$ for every $\alpha \in(0,1)$, then the function $G: D_{1} \rightarrow \mathbb{R}$ given by (6.1) belongs to $C^{1, \alpha^{\prime}}\left(D_{1}\right)$ for every $\alpha^{\prime} \in(0,2-2 s)$, and (6.12) holds.

Proof. We proceed as in the proof of Lemma 6.4 using notation (6.2). We fix an arbitrary point $x \in D_{1}$ and we want to show that $G$ admits a partial derivative $\partial_{i} G$ at $x$. For $t>0$ small, we have

$$
\begin{aligned}
\Gamma\left(x+t e_{i}, y\right)-\Gamma(x, y)= & \left(\int_{0}^{t}\left(\partial_{i} f\left(x+y+\rho e_{i}\right)-\partial_{i} f\left(x+\rho e_{i}\right)\right) \mathrm{d} \rho\right) \cdot\left(g\left(x+y+t e_{i}\right)-g\left(x+t e_{i}\right)\right) \\
& +(f(x+y)-f(x)) \cdot\left(\int_{0}^{t}\left(\partial_{i} g\left(x+y+\rho e_{i}\right)-\partial_{i} g\left(x+\rho e_{i}\right)\right) \mathrm{d} \rho\right)
\end{aligned}
$$


for every $y \in D_{1}$. Fixing an exponent $\alpha \in(2 s-1,1)$, we deduce that

$$
\left|\Gamma\left(x+t e_{i}, y\right)-\Gamma(x, y)\right| \leqslant C_{f, g, \alpha}|y|^{1+\alpha} t \quad \forall y \in D_{1} .
$$

Consequently,

$$
\frac{\left|\Gamma\left(x+t e_{i}, y\right)-\Gamma(x, y)\right|}{|y|^{n+2 s} t} \leqslant C_{f, g, \alpha}|y|^{1+\alpha-n-2 s} \in L^{1}\left(D_{1}\right) .
$$

As in the proof of Lemma 6.4, it now follows that $G$ admits a partial derivative $\partial_{i} G$ at $x$ given by (6.12), and the Hölder continuity of the partial derivatives of $G$ is a consequence of Lemma 6.3 .

\subsection{Hölder continuity of higher order derivatives.}

Proposition 6.6. Let $u \in \widehat{H}^{s}\left(D_{3} ; \mathbb{S}^{d-1}\right)$ be a weakly s-harmonic map in $D_{3}$. If $u \in C^{k, \alpha}\left(D_{3}\right)$ for some integer $k \geqslant 1$ and every $\alpha \in(0,1)$, then $u \in C^{k+1, \alpha}\left(D_{r_{*}}\right)$ for every $\alpha \in(0,1)$, where the radius $r_{*} \in(0,1 / 2)$ is given by Proposition 6.2.

Proof. We proceed as in Step 1 in the proof of Proposition 6.2, and we consider the function $G_{u}: D_{1} \rightarrow \mathbb{R}$ given by (6.7). We claim that $G_{u} \in C^{k, \alpha}\left(D_{1}\right)$ for every $\alpha \in(0,1)$ if $s \in(0,1 / 2)$, and that $G_{u} \in C^{k, \alpha}\left(D_{1}\right)$ for every $\alpha \in(0,2-2 s)$ if $s \in[1 / 2,1)$, together with the formula

$$
\partial^{\beta} G_{u}(x)=\sum_{\nu \leqslant \beta}\left(\begin{array}{l}
\beta \\
\nu
\end{array}\right) \int_{D_{1}} \frac{\left(\partial^{\nu} u(x+y)-\partial^{\nu} u(x)\right) \cdot\left(\partial^{\beta-\nu} u(x+y)-\partial^{\beta-\nu} u(x)\right)}{|y|^{n+2 s}} \zeta(y) \mathrm{d} y
$$

for every multi-index $\beta \in \mathbb{N}^{n}$ of length $|\beta| \leqslant k$. To prove this claim, we distinguish the case $s \in(0,1 / 2)$ from the case $s \in[1 / 2,1)$.

Case $s \in(0,1 / 2)$. We proceed by induction. First notice that the fact that $G_{u} \in C^{1, \alpha}\left(D_{1}\right)$ for every $\alpha \in(0,1)$ follows from Lemma 6.4 , as well as $(6.13)$ with $|\beta|=1$. Next we assume that $G_{u} \in C^{\ell, \alpha}\left(D_{1}\right)$ for every $\alpha \in(0,1)$ for some integer $\ell<k$, and that $(6.13)$ holds for every multi-index $\beta$ satisfying $|\beta|=\ell$. Applying Lemma 6.4 to each term in the right hand side of (6.13), we infer that $\partial^{\beta} G_{u} \in C^{1, \alpha}\left(D_{1}\right)$ for every $\alpha \in(0,1)$ and each $\beta$ satisfying $|\beta|=\ell$, and that (6.13) holds for multi-indices $\beta^{\prime}$ in place of $\beta$ of length $\left|\beta^{\prime}\right|=|\beta|+1$. The claim is thus proved for $s \in(0,1 / 2)$.

Case $s \in[1 / 2,1)$. We proceed exactly as in the previous case but using Lemma 6.5 instead of Lemma 6.4.

Now we consider the function $H_{u}: D_{1} \rightarrow \mathbb{R}$ given by (6.9) which clearly belongs to $C^{k, \alpha}\left(D_{1}\right)$ for every $\alpha \in(0,1)$ by our assumption on $u$. Consequently, the map $F_{u}: D_{1} \rightarrow \mathbb{R}^{d}$ belongs to $C^{k, \alpha}\left(D_{1}\right)$ for every $\alpha \in(0,1)$ if $s \in(0,1 / 2)$, and to $C^{k, \alpha}\left(D_{1}\right)$ for every $\alpha \in(0,2-2 s)$ if $s \in[1 / 2,1)$. By the results in [41] (together with Lemma 6.4 and Lemma 6.5), it implies that the map $u_{0}:=\zeta u$ as defined in Step 2, proof of Proposition 6.2, belongs to $C^{k+1, \alpha}\left(D_{1 / 2}\right)$ for every $\alpha \in(0,2 s)$ if $s \in(0,1 / 2)$, and to $C^{k+1, \alpha}\left(D_{1 / 2}\right)$ for every $\alpha \in(0,1)$ if $s \in[1 / 2,1)$. Since $u_{0}=u$ in $D_{1 / 2}$, the proof is thus complete for $s \in[1 / 2,1)$. In the case $s \in(0,1 / 2)$, we argue as in the proof of Proposition 6.2, Step 3, applying (inductively) Lemma 6.4 to formula (6.13) with $|\beta|=k$. It leads to the fact that $u \in C^{k+1, \alpha}\left(D_{r_{*}}\right)$ for every $\alpha \in(0,1)$, and hence concludes the proof.

\section{PARTIAL REgUlarity FOR STATIONARY AND MINIMIZING $s$-HARMONIC MAPS}

In this section, we complete the proof of Theorems 1.1, 1.2, and 1.3. For $n>2 s$, we need to prove compactness of stationary / minimizing $s$-harmonic map to apply Federer's dimension reduction principle. This is the object of the first subsection.

\subsection{Compactness properties of $s$-harmonic maps.}

Theorem 7.1. Assume that $s \in(0,1) \backslash\{1 / 2\}$ and $n>2 s$. Let $\Omega \subseteq \mathbb{R}^{n}$ be a bounded open set. Let $\left\{u_{k}\right\} \subseteq \widehat{H}^{s}\left(\Omega ; \mathbb{S}^{d-1}\right)$ be a sequence of stationary weakly s-harmonic maps in $\Omega$. Assume that $\sup _{k} \overline{\mathcal{E}}_{s}\left(u_{k}, \Omega\right)<+\infty$, and that $u_{k} \rightarrow$ u a.e. in $\mathbb{R}^{n}$. Then $u \in \widehat{H}^{s}\left(\Omega ; \mathbb{S}^{d-1}\right), u_{k} \rightarrow u$ weakly in $\widehat{H}^{s}\left(\Omega ; \mathbb{R}^{d}\right)$, and $u$ is a stationary weakly s-harmonic map in $\Omega$. In addition, for every open subset $\omega \subseteq \Omega$ and every bounded admissible open set $G \subseteq \mathbb{R}_{+}^{n+1}$ satisfying $\bar{\omega} \subseteq \Omega$ and $\overline{\partial^{0} G} \subseteq \Omega$,

(i) $u_{k} \rightarrow u$ strongly in $\widehat{H}^{s}\left(\omega ; \mathbb{R}^{d}\right)$; 
(ii) $u_{k}^{\mathrm{e}} \rightarrow u^{\mathrm{e}}$ strongly in $H^{1}\left(G ; \mathbb{R}^{d},|z|^{a} \mathrm{~d} \mathbf{x}\right)$.

Theorem 7.2. Assume that $s \in(0,1 / 2)$. In addition to Theorem 7.1 , if each $u_{k}$ is assumed to be a minimizing s-harmonic map in $\Omega$, then the limit $u$ is a minimizing s-harmonic map in $\Omega$.

Theorem 7.3. Let $\Omega \subseteq \mathbb{R}^{n}$ be a bounded open set and $\left\{u_{k}\right\} \subseteq \widehat{H}^{1 / 2}\left(\Omega ; \mathbb{S}^{d-1}\right)$ be a sequence of minimizing $1 / 2$-harmonic maps in $\Omega$. Assume that $\sup _{k} \mathcal{E}_{\frac{1}{2}}\left(u_{k}, \Omega\right)<+\infty$, and that $u_{k} \rightarrow$ u a.e. in $\mathbb{R}^{n}$. Then the conclusion of Theorem 7.1 holds and the limit $u$ is a minimizing $1 / 2$-harmonic map in $\Omega$.

Remark 7.4. In the case $s \in(1 / 2,1)$, we do not know if minimality of the sequence $\left\{u_{k}\right\}$ implies minimality of the limit. We believe this is indeed the case, but we won't need this fact.

Remark 7.5. In the case $n=1$ and $s \in(1 / 2,1)$, sequences of (arbitrary) weakly $s$-harmonic maps with uniformly bounded energy are relatively compact, i.e., the conclusion of Theorem 7.1 holds. This fact is a consequence of the Lipschitz estimate established in Theorem 5.1 together with Remark 4.3. Since we shall not need this, we leave the details to the reader.

Remark 7.6. In the case $s=1 / 2$, sequences of (stationary or not) $1 / 2$-harmonic maps are not compact in general, see e.g. $[6,31,35,30]$. The prototypical example is the following sequence of smooth $1 / 2$-harmonic maps from $\mathbb{R}^{n}$ into $\mathbb{S}^{1} \subseteq \mathbb{C}$ given by

$$
u_{k}(x)=u_{k}\left(x_{1}\right):=\frac{k x_{1}-i}{k x_{1}+i}, \quad k \in \mathbb{N},
$$

which is converging weakly but not strongly to the constant map 1 in $\widehat{H}^{1 / 2}\left(D_{r}\right)$ for every $r>0$. (Recall that $u_{k}$ being smooth, it is stationary, see Remark 3.7.)

Proof of Theorem 7.1. Step 1. We fix two arbitrary admissible bounded open $\operatorname{sets} G, G^{\prime} \subseteq \mathbb{R}_{+}^{n+1}$ such that $\bar{G} \subseteq G^{\prime} \cup \partial^{0} G^{\prime}$ and satisfying $\overline{\partial^{0} G^{\prime}} \subseteq \Omega$. Since $u_{k} \rightarrow u$ a.e. in $\mathbb{R}^{n}$ and $\left|u_{k}\right|=1$, we first deduce that $|u|=1$ and $u_{k} \rightarrow u$ strongly in $L_{\text {loc }}^{2}\left(\mathbb{R}^{2} ; \mathbb{R}^{d}\right)$. It then follows from our assumption that $\left\{u_{k}\right\}$ is bounded in $\widehat{H}^{s}\left(\Omega ; \mathbb{R}^{d}\right)$. Next we derive from Remark 2.2 that $u \in \widehat{H}^{s}\left(\Omega ; \mathbb{S}^{d-1}\right)$ and $u_{k} \rightarrow u$ weakly in $\widehat{H}^{s}\left(\Omega ; \mathbb{R}^{d}\right)$. In view of Corollary $2.10, u_{k}^{\mathrm{e}} \rightarrow u^{\mathrm{e}}$ weakly in $H^{1}\left(G^{\prime} ; \mathbb{R}^{d},|z|^{a} \mathrm{~d} \mathbf{x}\right)$. Since $\left|u_{k}\right| \leqslant 1$, we have $u_{k}^{\mathrm{e}}(\mathbf{x}) \rightarrow u^{\mathrm{e}}(\mathbf{x})$ for every $\mathbf{x} \in G^{\prime}$ by dominated convergence. In turn, we have $\left|u_{k}^{\mathrm{e}}-u^{\mathrm{e}}\right| \leqslant 2$, and it follows by dominated convergence again that $u_{k}^{\mathrm{e}} \rightarrow u^{\mathrm{e}}$ strongly in $L^{2}\left(G^{\prime} ; \mathbb{R}^{d},|z|^{a} \mathrm{~d} \mathbf{x}\right)$. Recalling that $\operatorname{div}\left(z^{a} \nabla u_{k}^{\mathrm{e}}\right)=0$ in $G^{\prime}$, we infer from standard elliptic regularity that $u_{k}^{\mathrm{e}} \rightarrow u^{\mathrm{e}}$ in $C_{\mathrm{loc}}^{1}\left(G^{\prime}\right)$. In particular,

$$
u_{k}^{\mathrm{e}} \rightarrow u^{\mathrm{e}} \quad \text { strongly in } H_{\mathrm{loc}}^{1}\left(G^{\prime} ; \mathbb{R}^{d}\right) \text {. }
$$

We aim to show that $u_{k}^{\mathrm{e}} \rightarrow u^{\mathrm{e}}$ strongly in $H^{1}\left(G ; \mathbb{R}^{d},|z|^{a} \mathrm{~d} \mathbf{x}\right)$. To prove this strong convergence, we consider the finite measures on $G^{\prime} \cup \partial^{0} G^{\prime}$ given by

$$
\mu_{k}:=\frac{\boldsymbol{\delta}_{s}}{2} z^{a}\left|\nabla u_{k}^{\mathrm{e}}\right|^{2} \mathscr{L}^{n+1}\left\llcorner G^{\prime} .\right.
$$

Since $\sup _{k} \mu_{k}\left(G^{\prime} \cup \partial^{0} G^{\prime}\right)<+\infty$, we can find a further (not relabeled) subsequence such that

$$
\mu_{k} \rightarrow \frac{\boldsymbol{\delta}_{s}}{2} z^{a}\left|\nabla u^{\mathrm{e}}\right|^{2} \mathscr{L}^{n+1}\left\llcorner G^{\prime}+\mu_{\text {sing }} \quad \text { as } k \rightarrow \infty,\right.
$$

weakly* as Radon measures on $G^{\prime} \cup \partial^{0} G^{\prime}$ for some finite nonnegative measure $\mu_{\text {sing. }}$. In view of (7.1), the defect measure $\mu_{\text {sing }}$ is supported by $\partial^{0} G^{\prime}$.

Since $u_{k}$ is stationary in $\Omega$, it satisfies the monotonicity formula in Proposition 2.17, and thus

$$
\frac{1}{\rho^{n-2 s}} \mu_{k}\left(B_{\rho}(\mathbf{x})\right) \leqslant \frac{1}{r^{n-2 s}} \mu_{k}\left(B_{r}(\mathbf{x})\right)
$$

for every $\mathbf{x} \in \partial^{0} G^{\prime}$ and $0<\rho<r<\operatorname{dist}\left(\mathbf{x}, \partial^{+} G^{\prime}\right)$. From the weak* convergence of $\mu_{k}$ towards $\mu$, we then infer that

$$
\frac{1}{\rho^{n-2 s}} \mu\left(B_{\rho}(\mathbf{x})\right) \leqslant \frac{1}{r^{n-2 s}} \mu\left(B_{r}(\mathbf{x})\right)
$$

for every $\mathbf{x} \in \partial^{0} G^{\prime}$ and $0<\rho<r<\operatorname{dist}\left(\mathbf{x}, \partial^{+} G^{\prime}\right)$. As a consequence, the $(n-2 s)$-dimensional density

$$
\Theta^{n-2 s}(\mu, \mathbf{x}):=\lim _{r \rightarrow 0} \frac{\mu\left(B_{r}(\mathbf{x})\right)}{r^{n-2 s}}
$$


exists and is finite at every point $\mathbf{x} \in \partial^{0} G^{\prime}$. More precisely, (7.3) implies that

$$
\Theta^{n-2 s}(\mu, \mathbf{x}) \leqslant\left(\operatorname{dist}\left(\mathbf{x}, \partial^{+} G^{\prime}\right)\right)^{2 s-n} \sup _{k} \mathbf{E}_{s}\left(u_{k}, G^{\prime}\right)<+\infty \quad \forall \mathbf{x} \in \partial^{0} G^{\prime} .
$$

We now consider the "concentration set"

$$
\Sigma:=\left\{\mathbf{x} \in \partial^{0} G^{\prime}: \inf _{r}\left\{\liminf _{k \rightarrow \infty} r^{2 s-n} \mu_{k}\left(B_{r}(\mathbf{x})\right): 0<r<\operatorname{dist}\left(\mathbf{x}, \partial^{+} G^{\prime}\right)\right\} \geqslant \varepsilon_{1}\right\},
$$

where the constant $\varepsilon_{1}>0$ is given by Corollary 4.2. From the monotonicity of $\mu_{k}$ and $\mu$ together with (7.2), we deduce that

$$
\begin{aligned}
\Sigma=\left\{\mathbf{x} \in \partial^{0} G^{\prime}: \lim _{r \rightarrow 0} \liminf _{k \rightarrow \infty} r^{2 s-n} \mu_{k}\left(B_{r}(\mathbf{x})\right) \geqslant\right. & \left.\varepsilon_{1}\right\} \\
& =\left\{\mathbf{x} \in \partial^{0} G^{\prime}: \lim _{r \rightarrow 0} r^{2 s-n} \mu\left(B_{r}(\mathbf{x})\right) \geqslant \varepsilon_{1}\right\},
\end{aligned}
$$

that is

$$
\Sigma=\left\{\mathbf{x} \in \partial^{0} G^{\prime}: \Theta^{n-2 s}(\mu, \mathbf{x}) \geqslant \varepsilon_{1}\right\} .
$$

Observing that $\mathbf{x} \in \partial^{0} G^{\prime} \mapsto \Theta^{n-2 s}(\mu, \mathbf{x})$ is upper semi-continuous, the set $\Sigma$ is a relatively closed subset of $\partial^{0} G^{\prime}$.

We claim that $\operatorname{spt}\left(\mu_{\text {sing }}\right) \subseteq \Sigma$. To prove this inclusion, we fix an arbitrary point $\mathbf{x}_{0}=\left(x_{0}, 0\right) \in$ $\partial^{0} G^{\prime} \backslash \Sigma$. Then we can find a radius $0<r<\operatorname{dist}\left(\mathbf{x}_{0}, \partial^{+} G^{\prime}\right)$ such that $r^{2 s-n} \mu\left(B_{r}\left(\mathbf{x}_{0}\right)\right)<\varepsilon_{1}$ and $\mu\left(\partial B_{r}\left(\mathbf{x}_{0}\right)\right)=0$. By (7.2) and our choice of $r$, we have $\lim _{k} \mu_{k}\left(B_{r}\left(\mathbf{x}_{0}\right)\right)=\mu\left(B_{r}\left(\mathbf{x}_{0}\right)\right)$. Therefore, $r^{2 s-n} \mu_{k}\left(B_{r}\left(\mathbf{x}_{0}\right)\right)<\varepsilon_{1}$ for $k$ large enough, and we derive from Theorem 5.1 that for $k$ large enough, $u_{k}$ is bounded in $C^{0,1}\left(D_{\kappa_{2} r}\left(x_{0}\right)\right)$ (and $u \in C^{0,1}\left(D_{\boldsymbol{\kappa}_{2} r}\left(x_{0}\right)\right)$ ), where the constant $\boldsymbol{\kappa}_{2} \in(0,1)$ only depends on $n$ and $s$. It then follows by dominated convergence that

$$
\left[u_{k}-u\right]_{H^{s}\left(D_{\kappa_{2} r}\left(x_{0}\right)\right)}^{2} \underset{k \rightarrow \infty}{\longrightarrow} 0 .
$$

Setting $w_{k}:=u_{k}-u$, we now estimate

$$
\begin{aligned}
& \mathcal{E}_{s}\left(w_{k}, D_{2 \kappa_{2} r / 3}\left(x_{0}\right)\right) \leqslant C\left(\left[u_{k}-u\right]_{H^{s}\left(D_{\kappa_{2} r}\left(x_{0}\right)\right)}^{2}\right. \\
& \left.\quad+\iint_{D_{2 \kappa_{2} r / 3}\left(x_{0}\right) \times D_{\kappa_{2} r}^{c}\left(x_{0}\right)} \frac{\left|w_{k}(x)-w_{k}(y)\right|^{2}}{|x-y|^{n+2 s}} \mathrm{~d} x \mathrm{~d} y\right) .
\end{aligned}
$$

Since $\left|w_{k}\right| \leqslant 2$ and $w_{k} \rightarrow 0$ a.e. in $\mathbb{R}^{n}$, by dominated convergence we have

$$
\iint_{D_{2 \kappa_{2} r / 3}\left(x_{0}\right) \times D_{\kappa_{2} r}^{c}\left(x_{0}\right)} \frac{\left|w_{k}(x)-w_{k}(y)\right|^{2}}{|x-y|^{n+2 s}} \mathrm{~d} x \mathrm{~d} y \underset{k \rightarrow \infty}{\longrightarrow} 0 .
$$

Hence $\mathcal{E}_{s}\left(w_{k}, D_{2 \kappa_{2} r / 3}\left(x_{0}\right)\right) \rightarrow 0$, and it follows from Lemma 2.9 that

$$
\mathbf{E}_{s}\left(u_{k}^{\mathrm{e}}-u^{\mathrm{e}}, B_{\boldsymbol{\kappa}_{2} r / 3}^{+}\left(\mathbf{x}_{0}\right)\right) \leqslant C \mathcal{E}_{s}\left(u_{k}-u, D_{2 \boldsymbol{\kappa}_{2} r / 3}\left(x_{0}\right)\right) \rightarrow 0 .
$$

Hence, $u_{k}^{\mathrm{e}} \rightarrow u^{\mathrm{e}}$ strongly in $H^{1}\left(B_{\boldsymbol{\kappa}_{2} r / 3}^{+}\left(\mathbf{x}_{0}\right),|z|^{a} \mathrm{~d} \mathbf{x}\right)$, and thus $\mu_{\text {sing }}\left(B_{\boldsymbol{\kappa}_{2} r / 3}\left(\mathbf{x}_{0}\right)\right)=0$. This shows that $\mathbf{x}_{0} \notin \operatorname{spt}\left(\mu_{\text {sing }}\right)$, and the claim is proved.

Next we claim that $\mu(\Sigma)=0$. Indeed, assume by contradiction that $\mu(\Sigma)>0$. Then the density $\Theta^{n-2 s}(\mu, \mathbf{x})$ exists, it is positive (greater than $\varepsilon_{1}$ ) and finite, at every point $\mathbf{x} \in \Sigma$. By Marstrand's theorem (see e.g. [28, Theorem 14.10]), it implies that $n-2 s$ is an integer, a contradiction.

Knowing that $\mu(\Sigma)=0$, we now deduce that $\mu_{\text {sing }}(\Sigma)=0$. But $\mu_{\text {sing }}$ being supported by $\Sigma$, it implies that $\mu_{\text {sing }} \equiv 0$. As a consequence, $\mathbf{E}_{s}\left(u_{k}^{\mathrm{e}}, G\right) \rightarrow \mathbf{E}_{s}\left(u^{\mathrm{e}}, G\right)$, which combined with the weak convergence in $H^{1}\left(G ; \mathbb{R}^{d},|z|^{a} \mathrm{~d} \mathbf{x}\right)$ implies that $\mathbf{E}_{s}\left(u_{k}^{\mathrm{e}}-u^{\mathrm{e}}, G\right) \rightarrow 0$. We have thus proved that $u_{k}^{\mathrm{e}} \rightarrow u^{\mathrm{e}}$ strongly in $H^{1}\left(G ; \mathbb{R}^{d},|z|^{a} \mathrm{~d} \mathbf{x}\right)$.

Step 2. We consider in this step an open subset $\omega \subseteq \Omega$ such that $\bar{\omega} \subseteq \Omega$, and our goal is to prove that $u_{k} \rightarrow u$ strongly in $\widehat{H}^{s}\left(\omega ; \mathbb{R}^{d}\right)$. Set $\delta:=\frac{1}{8} \operatorname{dist}\left(\omega, \Omega^{c}\right)$, and consider a finite covering of $\omega$ by balls $\left(D_{\delta}\left(x_{i}\right)\right)_{i \in I}$ with $x_{i} \in \bar{\omega}$. By Lemma 2.8 and Step 1, we have for each $i \in I$,

$$
\left[u_{k}-u\right]_{H^{s}\left(D_{2 \delta}\left(x_{i}\right)\right)}^{2} \leqslant C \mathbf{E}_{s}\left(u_{k}^{\mathrm{e}}-u^{\mathrm{e}}, B_{4 \delta}^{+}\left(\mathbf{x}_{i}\right)\right) \underset{k \rightarrow \infty}{\longrightarrow} 0,
$$


where $\mathbf{x}_{i}:=\left(x_{i}, 0\right)$. Writing again $w_{k}:=u_{k}-u$, we now estimate

$$
\begin{aligned}
\mathcal{E}_{s}\left(w_{k}, \omega\right) & \leqslant C \iint_{\omega \times \mathbb{R}^{n}} \frac{\left|w_{k}(x)-w_{k}(y)\right|^{2}}{|x-y|^{n+2 s}} \mathrm{~d} x \mathrm{~d} y \\
& \leqslant C \sum_{i \in I} \iint_{D_{\delta}\left(x_{i}\right) \times \mathbb{R}^{n}} \frac{\left|w_{k}(x)-w_{k}(y)\right|^{2}}{|x-y|^{n+2 s}} \mathrm{~d} x \mathrm{~d} y \\
& \leqslant C \sum_{i \in I}\left(\left[w_{k}\right]_{H^{s}\left(D_{2 \delta}\left(x_{i}\right)\right)}^{2}+\iint_{D_{\delta}\left(x_{i}\right) \times D_{2 \delta}^{c}\left(x_{i}\right)} \frac{\left|w_{k}(x)-w_{k}(y)\right|^{2}}{|x-y|^{n+2 s}} \mathrm{~d} x \mathrm{~d} y\right) .
\end{aligned}
$$

As in (7.4), by dominated convergence we have

$$
\iint_{D_{\delta}\left(x_{i}\right) \times D_{2 \delta}^{c}\left(x_{i}\right)} \frac{\left|w_{k}(x)-w_{k}(y)\right|^{2}}{|x-y|^{n+2 s}} \mathrm{~d} x \mathrm{~d} y \underset{k \rightarrow \infty}{\longrightarrow} 0 \quad \forall i \in I .
$$

Combining (7.5), (7.6), and (7.7) leads to $\mathcal{E}_{s}\left(w_{k}, \omega\right) \rightarrow 0$, and thus $u_{k} \rightarrow u$ strongly in $\widehat{H}^{s}\left(\omega ; \mathbb{R}^{d}\right)$.

Step 3. Our aim in this step is to show that $u$ is a weakly $s$-harmonic map in $\Omega$, i.e., $u$ satisfies equation (3.2), or equivalently (3.4), by Proposition 3.5. To this purpose, we fix an arbitrary $\varphi \in \mathscr{D}\left(\Omega ; \mathbb{R}^{d}\right)$, and we choose an open subset $\omega \subseteq \Omega$ such that $\operatorname{spt}(\varphi) \subseteq \omega$ and $\bar{\omega} \subseteq \Omega$. Writing again $w_{k}:=u_{k}-u$, we have proved in Step 2 that $\mathcal{E}_{s}\left(w_{k}, \omega\right) \rightarrow 0$.

Recalling our notations from Subsection 2.2, we observe that

$$
\left|\mathrm{d}_{s} u_{k}\right|^{2}-\left|\mathrm{d}_{s} u\right|^{2}=\left|\mathrm{d}_{s} w_{k}\right|^{2}+2 \mathrm{~d}_{s} w_{k} \odot \mathrm{d}_{s} u
$$

and then estimate

$$
\begin{aligned}
\left\|\left|\mathrm{d}_{s} u_{k}\right|^{2}-\left|\mathrm{d}_{s} u\right|^{2}\right\|_{L^{1}(\omega)} & \leqslant\left\|\left|\mathrm{d}_{s} w_{k}\right|^{2}\right\|_{L^{1}(\omega)}+2\left\|\mathrm{~d}_{s} w_{k} \odot \mathrm{d}_{s} u\right\|_{L^{1}(\omega)} \\
& \leqslant 2 \mathcal{E}_{s}\left(w_{k}, \omega\right)+2\left\|\mathrm{~d}_{s} w_{k}\right\|_{L_{\text {od }}^{2}(\omega)}\left\|\mathrm{d}_{s} u\right\|_{L_{\text {od }}^{2}(\omega)} \\
& \leqslant 2 \mathcal{E}_{s}\left(w_{k}, \omega\right)+2 \sqrt{2}\left\|\mathrm{~d}_{s} u\right\|_{L_{\text {od }}^{2}(\omega)} \sqrt{\mathcal{E}_{s}\left(w_{k}, \omega\right)} .
\end{aligned}
$$

Therefore $\left|\mathrm{d}_{s} u_{k}\right|^{2} \rightarrow\left|\mathrm{d}_{s} u\right|^{2}$ in $L^{1}(\omega)$, and we can find a further (not relabeled) subsequence and $h \in L^{1}(\omega)$ such that

$$
\left|\mathrm{d}_{s} u_{k}\right|^{2}(x) \rightarrow\left|\mathrm{d}_{s} u\right|^{2}(x) \text { for a.e. } x \in \omega, \text { and }\left|\mathrm{d}_{s} u_{k}\right|^{2}(x) \leqslant h(x) \text { for a.e. } x \in \omega .
$$

Since $\left|u_{k}\right|=1$ and $u_{k} \rightarrow u$ a.e. in $\omega$, it follows by dominated convergence that $\left|\mathrm{d}_{s} u_{k}\right|^{2} u_{k} \rightarrow$ $\left|\mathrm{d}_{s} u\right|^{2} u$ in $L^{1}(\omega)$. Consequently,

$$
\int_{\Omega}\left|\mathrm{d}_{s} u_{k}\right|^{2} u_{k} \cdot \varphi \mathrm{d} x \underset{k \rightarrow \infty}{\longrightarrow} \int_{\Omega}\left|\mathrm{d}_{s} u\right|^{2} u \cdot \varphi \mathrm{d} x .
$$

On the other hand, the weak convergence of $u_{k}$ to $u$ in $\widehat{H}^{s}\left(\Omega ; \mathbb{R}^{d}\right)$ implies that $\left\langle(-\Delta)^{s} u_{k}, \varphi\right\rangle_{\Omega}$ converges to $\left\langle(-\Delta)^{s} u, \varphi\right\rangle_{\Omega}$. Hence,

$$
\left\langle(-\Delta)^{s} u, \varphi\right\rangle_{\Omega}=\lim _{k \rightarrow \infty}\left\langle(-\Delta)^{s} u_{k}, \varphi\right\rangle_{\Omega}=\lim _{k \rightarrow \infty} \int_{\Omega}\left|\mathrm{d}_{s} u_{k}\right|^{2} u_{k} \cdot \varphi \mathrm{d} x=\int_{\Omega}\left|\mathrm{d}_{s} u\right|^{2} u \cdot \varphi \mathrm{d} x,
$$

so that $u$ is indeed weakly $s$-harmonic in $\Omega$ (see (3.4)).

Step 4. It now only remains to prove that $u$ is stationary in $\Omega$. This is in fact an easy consequence of the strong convergence of $u^{\mathrm{e}}$ established in Step 1. Indeed, let us fix an arbitrary vector field $X \in C^{1}\left(\mathbb{R}^{n} ; \mathbb{R}^{n}\right)$ compactly supported in $\Omega$. Combining the strong convergence of $u_{k}^{\text {e }}$ established in Step 1 together with the representation of the first variation $\delta \mathcal{E}_{s}$ stated in Proposition 2.15, we obtain that $\delta \mathcal{E}_{s}\left(u_{k}, \Omega\right)[X] \rightarrow \delta \mathcal{E}_{s}(u, \Omega)[X]$, whence $\delta \mathcal{E}_{s}(u, \Omega)=0$.

Proof of Theorem 7.2. In view of Remark 3.3 and Theorem 7.1, it only remains to prove that the limiting map $u$ is a minimizing $s$-harmonic map in $\Omega$. We follow here the argument in [34, Theorem 4.1]

Let us now consider an arbitrary $\widetilde{u} \in \widehat{H}^{s}\left(\Omega ; \mathbb{S}^{d-1}\right) \operatorname{such}$ that $\operatorname{spt}(u-\widetilde{u}) \subseteq \Omega$. We select an open subset $\omega \subseteq \Omega$ with Lipschitz boundary such that $\operatorname{spt}(u-\widetilde{u}) \subseteq \omega$ and $\bar{\omega} \subseteq \Omega$. Define

$$
\widetilde{u}_{k}(x):= \begin{cases}\widetilde{u}(x) & \text { if } x \in \omega \\ u_{k}(x) & \text { otherwise }\end{cases}
$$


Since $s \in(0,1 / 2)$ and $\partial \omega$ is Lipschitz regular, it turns out that $\widetilde{u}_{k} \in \widehat{H}^{s}\left(\Omega ; \mathbb{S}^{d-1}\right)$ (see e.g. [33, Section 2.1]), and $\operatorname{spt}\left(u_{k}-\widetilde{u}_{k}\right) \subseteq \Omega$. By minimality of $u_{k}$, we have $\mathcal{E}_{s}\left(u_{k}, \Omega\right) \leqslant \mathcal{E}_{s}\left(\widetilde{u}_{k}, \Omega\right)$. Since $\widetilde{u}_{k}=u_{k}$ in $\mathbb{R}^{n} \backslash \omega$, it reduces to

$$
\mathcal{E}_{s}\left(u_{k}, \omega\right) \leqslant \mathcal{E}_{s}\left(\widetilde{u}_{k}, \omega\right)=\frac{\gamma_{n, s}}{4} \iint_{\omega \times \omega} \frac{|\widetilde{u}(x)-\widetilde{u}(y)|^{2}}{|x-y|^{n+2 s}} \mathrm{~d} x \mathrm{~d} y+\frac{\gamma_{n, s}}{2} \iint_{\omega \times \omega^{c}} \frac{\left|\widetilde{u}(x)-u_{k}(y)\right|^{2}}{|x-y|^{n+2 s}} \mathrm{~d} x \mathrm{~d} y .
$$

On the other hand,

$$
\frac{\left|\widetilde{u}(x)-u_{k}(y)\right|^{2}}{|x-y|^{n+2 s}} \leqslant \frac{4}{|x-y|^{n+2 s}} \in L^{1}\left(\omega \times \omega^{c}\right),
$$

since $\omega$ has Lipschitz boundary. Hence, $\mathcal{E}_{s}\left(\widetilde{u}_{k}, \omega\right) \rightarrow \mathcal{E}_{s}(\widetilde{u}, \omega)$ by dominated convergence and the fact that $\widetilde{u}=u$ in $\mathbb{R}^{n} \backslash \omega$. By Fatou's Lemma, we have $\liminf _{k} \mathcal{E}_{s}\left(u_{k}, \omega\right) \geqslant \mathcal{E}_{s}(u, \omega)$, and we reach the conclusion that $\mathcal{E}_{s}(u, \omega) \leqslant \mathcal{E}_{s}(\widetilde{u}, \omega)$. Once again, the fact that $\widetilde{u}=u$ in $\mathbb{R}^{n} \backslash \omega$ then implies that $\mathcal{E}_{s}(u, \Omega) \leqslant \mathcal{E}_{s}(\widetilde{u}, \Omega)$. By arbitrariness of $\widetilde{u}$, we conclude that $u$ is indeed a minimizing $s$-harmonic map in $\Omega$.

Proof of Theorem 7.3. Step 1. Once again, we fix two arbitrary admissible bounded open sets $G, G^{\prime} \subseteq \mathbb{R}_{+}^{n+1}$ such that $\bar{G} \subseteq G^{\prime} \cup \partial^{0} G^{\prime}$ and satisfying $\overline{\partial^{0} G^{\prime}} \subseteq \Omega$. Exactly as in the beginning of the proof of Theorem 7.1, Step 1, we have: (i) $u_{k} \rightarrow u$ strongly in $L_{\text {loc }}^{2}\left(\mathbb{R}^{2} ; \mathbb{R}^{d}\right.$ ); (ii) $u_{k} \rightarrow u$ weakly in $\widehat{H}^{1 / 2}\left(\Omega ; \mathbb{R}^{d}\right)$; (iii) $u_{k}^{\mathrm{e}} \rightarrow u^{\mathrm{e}}$ strongly in $L^{2}\left(G^{\prime} ; \mathbb{R}^{d}\right) ;($ iv $) u_{k}^{\mathrm{e}} \rightarrow u^{\mathrm{e}}$ weakly in $H^{1}\left(G^{\prime} ; \mathbb{R}^{d}\right)$.

Since each $u_{k}$ is minimizing, we infer from [30, Theorem 3.6] that each $u_{k}^{\mathrm{e}}$ is a minimizing harmonic map with (partially) free boundary in $G^{\prime}$, i.e.,

$$
\mathbf{E}_{\frac{1}{2}}\left(u_{k}^{\mathrm{e}}, G^{\prime}\right) \leqslant \mathbf{E}_{\frac{1}{2}}\left(v, G^{\prime}\right)
$$

for every $v \in H^{1}\left(G^{\prime} ; \mathbb{R}^{d}\right)$ satisfying $v(\mathbf{x}) \in \mathbb{S}^{d-1}$ a.e. on $\partial^{0} G^{\prime}$, and $\operatorname{spt}\left(v-u_{k}^{\mathrm{e}}\right) \subseteq G^{\prime} \cup \partial^{0} G^{\prime}$ (see e.g. [30, Definition 3.1]). Applying [30, Theorem 3.5] (which is based on [13, 14]), we conclude that $u_{k}^{\mathrm{e}} \rightarrow u^{\mathrm{e}}$ strongly in $H_{\mathrm{loc}}^{1}\left(G^{\prime} \cup \partial^{0} G^{\prime} ; \mathbb{R}^{d}\right)$, and that $u^{\mathrm{e}}$ is a minimizing harmonic map with (partially) free boundary in $G^{\prime}$. In particular, $u_{k}^{\mathrm{e}} \rightarrow u^{\mathrm{e}}$ strongly in $H^{1}\left(G ; \mathbb{R}^{d}\right)$.

Step 2. In view of the arbitrariness of $G^{\prime}$, we first deduce that $u^{\mathrm{e}}$ is a minimizing harmonic map with (partially) free boundary in every admissible bounded open set $G^{\prime} \subseteq \mathbb{R}_{+}^{n+1}$ such that $\overline{\partial^{0} G^{\prime}} \subseteq \Omega$. By [30, Theorem 3.6], it implies that $u$ is a minimizing $1 / 2$-harmonic map in $\Omega$.

It now only remains to show that $u_{k} \rightarrow u$ strongly in $\widehat{H}^{1 / 2}\left(\omega ; \mathbb{R}^{d}\right)$, where $\omega \subseteq \Omega$ is an open subset satisfying $\bar{\omega} \subseteq \Omega$. Applying Lemma 2.8 and Step 1, the strong convergence in $\widehat{H}^{1 / 2}\left(\omega ; \mathbb{R}^{d}\right)$ can be achieved exactly as in the proof of Theorem 7.1 , Step 2 .

We now close this subsection with an easy consequence of Theorem 7.1 and Theorem 7.3 in terms of the pointwise density function $\boldsymbol{\Xi}_{s}(u, \cdot)$ defined in $(2.25)$.

Corollary 7.7. Assume that $n>2 s$. In addition to Theorem 7.1 and Theorem 7.3, if $\left\{x_{k}\right\} \subseteq \Omega$ is a sequence converging to $x_{*} \in \Omega$, then

$$
\limsup _{k \rightarrow \infty} \boldsymbol{\Xi}_{s}\left(u_{k}, x_{k}\right) \leqslant \boldsymbol{\Xi}_{s}\left(u, x_{*}\right) .
$$

Proof. Without loss of generality, we can assume that $x_{*}=0$. Applying Corollary 2.18, we obtain for $r>0$ small enough and $r_{k}:=\left|x_{k}\right|$,

$$
\boldsymbol{\Xi}_{s}\left(u_{k}, x_{k}\right) \leqslant \boldsymbol{\Theta}\left(u_{k}^{\mathrm{e}}, \mathbf{x}_{k}, r\right) \leqslant \frac{1}{r^{n-2 s}} \mathbf{E}_{s}\left(u_{k}^{\mathrm{e}}, B_{r+r_{k}}^{+}\right)
$$

where $\mathbf{x}_{k}:=\left(x_{k}, 0\right)$. By Theorem 7.1 (in the case $s \neq 1 / 2$ ) and Theorem 7.3 (in the case $s=1 / 2), u_{k}^{\mathrm{e}} \rightarrow u^{\mathrm{e}}$ strongly in $H^{1}\left(B_{2 r}^{+},|z|^{a} \mathrm{~d} \mathbf{x}\right)$. Since $r_{k} \rightarrow 0$, we deduce from (7.8) that

$$
\limsup _{k \rightarrow \infty} \boldsymbol{\Xi}_{s}\left(u_{k}, x_{k}\right) \leqslant \boldsymbol{\Theta}\left(u^{\mathrm{e}}, 0, r\right),
$$

and the conclusion follows letting $r \rightarrow 0$. 
7.2. Tangent maps. We assume throughout this subsection that $s \in(0,1)$ and $n>2 s$. We consider a bounded open set $\Omega \subseteq \mathbb{R}^{n}$ and a map $u \in \widehat{H}^{s}\left(\Omega ; \mathbb{S}^{d-1}\right)$ that we assume to be

- a stationary weakly $s$-harmonic map in $\Omega$ for $s \neq 1 / 2$;

- a minimizing $1 / 2$-harmonic map in $\Omega$ for $s=1 / 2$.

We shall apply the results of Subsection 7.1 to define the so-called tangent maps of $u$ at a given point. To this purpose, we fix a point of study $x_{0} \in \Omega$ and a reference radius $\rho_{0}>0$ such that $D_{3 \rho_{0}}\left(x_{0}\right) \subseteq \Omega$. We introduce the rescaled function

$$
u_{x_{0}, \rho}(x):=u\left(x_{0}+\rho x\right),
$$

and we observe that $\left(u_{x_{0}, \rho}\right)^{\mathrm{e}}(\mathbf{x})=u^{\mathrm{e}}\left(\mathbf{x}_{0}+\rho \mathbf{x}\right)=u_{x_{0}, \rho}^{\mathrm{e}}(\mathbf{x})$ with $\mathbf{x}_{0}=\left(x_{0}, 0\right)$. Rescaling variables, $u_{x_{0}, \rho}$ is a stationary weakly $s$-harmonic map in $\left(\Omega-x_{0}\right) / \rho$ for $s \neq 1 / 2$, or a minimizing $1 / 2$ harmonic map in $\left(\Omega-x_{0}\right) / \rho$ for $s=1 / 2$. In addition,

$$
\boldsymbol{\Theta}_{s}\left(u_{x_{0}, \rho}^{\mathrm{e}}, 0, r\right)=\boldsymbol{\Theta}_{s}\left(u^{\mathrm{e}}, \mathbf{x}_{0}, \rho r\right) \quad \forall r \in\left(0, \rho_{0} / \rho\right] .
$$

This identity together with the monotonicity formula in Proposition 2.17 and Lemma 2.9 yields

$$
\boldsymbol{\Theta}_{s}\left(u_{x_{0}, \rho}^{\mathrm{e}}, 0, r\right) \leqslant \boldsymbol{\Theta}_{s}\left(u^{\mathrm{e}}, \mathbf{x}_{0}, \rho_{0}\right) \leqslant C \rho_{0}^{2 s-n} \mathcal{E}_{s}(u, \Omega) \quad \forall r \in\left(0, \rho_{0} / \rho\right],
$$

for a constant $C$ depending only on $n$ and $s$. In turn, Lemma 2.8 implies that

$$
\left[u_{x_{0}, \rho}\right]_{H^{s}\left(D_{2 r}\right)}^{2} \leqslant C \rho_{0}^{2 s-n} r^{n-2 s} \mathcal{E}_{s}(u, \Omega) \quad \forall r \in\left(0, \rho_{0} /(4 \rho)\right] .
$$

Using $\left|u_{x_{0}, \rho}\right|=1$, we can now estimate for $r \in\left(0, \rho_{0} /(4 \rho)\right]$,

$$
\mathcal{E}_{s}\left(u_{x_{0}, \rho}, D_{r}\right) \leqslant C\left(\left[u_{x_{0}, \rho}\right]_{H^{s}\left(D_{2 r}\right)}^{2}+\iint_{D_{r} \times D_{2 r}^{c}} \frac{\mathrm{d} x \mathrm{~d} y}{|x-y|^{n+2 s}}\right) \leqslant C r^{n-2 s}\left(\rho_{0}^{2 s-n} \mathcal{E}_{s}(u, \Omega)+1\right) .
$$

Given a sequence $\rho_{k} \rightarrow 0$, we deduce from the above estimate that

$$
\limsup _{k \rightarrow \infty} \mathcal{E}_{s}\left(u_{x_{0}, \rho_{k}}, D_{r}\right)<+\infty \quad \forall r>0 .
$$

Applying Theorem 7.1, Theorem 7.2, and Theorem 7.3, we can now find a subsequence $\left\{\rho_{k}^{\prime}\right\}$ and $\varphi \in H_{\mathrm{loc}}^{s}\left(\mathbb{R}^{n} ; \mathbb{S}^{d-1}\right)$ such that

$u_{x_{0}, \rho_{k}^{\prime}} \rightarrow \varphi$ strongly in $\widehat{H}^{s}\left(D_{r}\right)$, and $u_{x_{0}, \rho_{k}^{\prime}}^{\mathrm{e}} \rightarrow \varphi^{\mathrm{e}}$ strongly in $H^{1}\left(B_{r}^{+},|z|^{a} \mathrm{~d} \mathbf{x}\right)$ for all $r>0$, where

(i) if $s \neq 1 / 2: \varphi$ is a stationary weakly $s$-harmonic map in $D_{r}$ for all $r>0$;

(ii) if $s \leqslant 1 / 2$ and $u$ minimizing: $\varphi$ is a minimizing $s$-harmonic map in $D_{r}$ for all $r>0$.

Definition 7.8. Every function $\varphi$ obtained by this process will be referred to as a tangent map to $u$ at the point $x_{0}$. The family of all tangent maps to $u$ at $x_{0}$ is denoted by $T_{x_{0}}(u)$.

We now present some classical properties of tangent maps following e.g. [53] or [33, Section 6].

Lemma 7.9. If $\varphi \in T_{x_{0}}(u)$, then

$$
\boldsymbol{\Theta}_{s}\left(\varphi^{\mathrm{e}}, 0, r\right)=\boldsymbol{\Xi}_{s}(\varphi, 0)=\boldsymbol{\Xi}_{s}\left(u, x_{0}\right) \quad \forall r>0,
$$

and $\varphi$ is positively 0 -homogeneous, i.e., $\varphi(\lambda x)=\varphi(x)$ for every $\lambda>0$ and $x \in \mathbb{R}^{n}$. In particular,

$$
\boldsymbol{\Xi}_{s}(\varphi, \lambda x)=\boldsymbol{\Xi}_{s}(\varphi, x) \text { for every } x \in \mathbb{R}^{n} \backslash\{0\} \text { and } \lambda>0 .
$$

Proof. From the strong convergence of $u_{x_{0}, \rho_{k}^{\prime}}^{\mathrm{e}}$ to $\varphi^{\mathrm{e}}$ in $H^{1}\left(B_{r}^{+},|z|^{a} \mathrm{dx}\right)$ and (7.9), we first deduce that

$$
\boldsymbol{\Theta}_{s}\left(\varphi^{\mathrm{e}}, 0, r\right)=\lim _{k \rightarrow \infty} \boldsymbol{\Theta}_{s}\left(u^{\mathrm{e}}, \mathbf{x}_{0}, \rho_{k}^{\prime} r\right)=\boldsymbol{\Xi}_{s}\left(u, x_{0}\right) \quad \forall r>0 .
$$

Then, the constancy of $r \mapsto \boldsymbol{\Theta}_{s}\left(\varphi^{\mathrm{e}}, 0, r\right)$ together with the monotonicity formula in Proposition 2.17 implies that $\mathbf{x} \cdot \nabla \varphi^{\mathrm{e}}(\mathbf{x})=0$ for every $\mathbf{x} \in \mathbb{R}_{+}^{n+1}$. Hence, $\varphi^{\mathrm{e}}$ is positively 0-homogeneous, and the homogeneity of $\varphi$ follows. As a consequence, for $x \in \mathbb{R}^{n} \backslash\{0\}$ and $\lambda>0$,

$$
\boldsymbol{\Theta}_{s}\left(\varphi^{\mathrm{e}}, \lambda \mathbf{x}, r\right)=\boldsymbol{\Theta}_{s}\left(\varphi^{\mathrm{e}}, \mathbf{x}, r / \lambda\right),
$$

where $\mathbf{x}:=(x, 0)$. Letting now $r \rightarrow 0$ yields (7.10). 
Lemma 7.10. If $\varphi \in T_{x_{0}}(u)$, then

$$
\mathbf{\Xi}_{s}(\varphi, y) \leqslant \boldsymbol{\Xi}_{s}(\varphi, 0) \quad \forall y \in \mathbb{R}^{n} .
$$

In addition, the set

$$
S(\varphi):=\left\{y \in \mathbb{R}^{n}: \boldsymbol{\Xi}_{s}(\varphi, y)=\mathbf{\Xi}_{s}(\varphi, 0)\right\}
$$

is a linear subspace of $\mathbb{R}^{n}$, and $\varphi(x+y)=\varphi(x)$ for every $y \in S(\varphi)$ and every $x \in \mathbb{R}^{n}$.

Proof. Step 1. By Corollary 2.18, we have have for every $y \in \mathbb{R}^{n}$ and $\rho>0$,

$$
\boldsymbol{\Xi}_{s}(\varphi, y)+\boldsymbol{\delta}_{s} \int_{B_{\rho}^{+}(\mathbf{y})} z^{a} \frac{\left|(\mathbf{x}-\mathbf{y}) \cdot \nabla \varphi^{\mathrm{e}}\right|^{2}}{|\mathbf{x}-\mathbf{y}|^{n+2-2 s}} \mathrm{~d} \mathbf{x}=\boldsymbol{\Theta}_{s}\left(\varphi^{\mathrm{e}}, \mathbf{y}, \rho\right)
$$

where $\mathbf{y}=(y, 0)$. On the other hand, by homogeneity of $\varphi$,

$$
\boldsymbol{\Theta}_{s}\left(\varphi^{\mathrm{e}}, \mathbf{y}, \rho\right) \leqslant \frac{(\rho+|y|)^{n-2 s}}{\rho^{n-2 s}} \boldsymbol{\Theta}_{s}\left(\varphi^{\mathrm{e}}, 0, \rho+|y|\right)=\frac{(\rho+|y|)^{n-2 s}}{\rho^{n-2 s}} \boldsymbol{\Xi}_{s}(\varphi, 0) .
$$

Combining this inequality with (7.11) and letting $\rho \rightarrow \infty$ yields

$$
\boldsymbol{\Xi}_{s}(\varphi, y)+\boldsymbol{\delta}_{s} \int_{\mathbb{R}_{+}^{n+1}} z^{a} \frac{\left|(\mathbf{x}-\mathbf{y}) \cdot \nabla \varphi^{\mathrm{e}}(\mathbf{x})\right|^{2}}{|\mathbf{x}-\mathbf{y}|^{n+2-2 s}} \mathrm{~d} \mathbf{x} \leqslant \boldsymbol{\Xi}_{s}(\varphi, 0) .
$$

Step 2. Next, assume that $\boldsymbol{\Xi}_{s}(\varphi, y)=\boldsymbol{\Xi}_{s}(\varphi, 0)$ for some $y \neq 0$. Then $(\mathbf{x}-\mathbf{y}) \cdot \nabla \varphi^{\mathrm{e}}(\mathbf{x})=0$ for all $\mathbf{x} \in \mathbb{R}_{+}^{n+1}$. By 0 -homogeneity of $\varphi^{\mathrm{e}}$, we then have $\mathbf{y} \cdot \nabla \varphi^{\mathrm{e}}(\mathbf{x})=0$ for all $\mathbf{x} \in \mathbb{R}_{+}^{n+1}$, and thus

$$
\varphi(x+y)=\varphi(x) \quad \forall x \in \mathbb{R}^{n} .
$$

The other way around, if (7.12) holds for some $y \neq 0$, then $(\mathbf{x}-\mathbf{y}) \cdot \nabla \varphi^{\mathrm{e}}(\mathbf{x})=0$ for all $\mathbf{x} \in \mathbb{R}_{+}^{n+1}$ (again by homogeneity). We then infer from (7.11) and (7.12) that for $\rho>0$,

$$
\boldsymbol{\Xi}_{s}(\varphi, y)=\boldsymbol{\Theta}_{s}\left(\varphi^{\mathrm{e}}, \mathbf{y}, \rho\right)=\boldsymbol{\Theta}_{s}\left(\varphi^{\mathrm{e}}, 0, \rho\right)=\boldsymbol{\Xi}_{s}(\varphi, 0),
$$

i.e., $y \in S(\varphi)$. Hence, (7.12) caracterizes $S(\varphi)$, and the linearity of $S(\varphi)$ follows.

Remark 7.11. If there exists $\varphi \in T_{x_{0}}(u)$ such that $\operatorname{dim} S(\varphi)=n$, then $\varphi$ is clearly constant, and thus $\boldsymbol{\Xi}_{s}\left(u, x_{0}\right)=\boldsymbol{\Xi}_{s}(\varphi, 0)=0$. By Theorem 5.1, $u$ is continuous in a neighborhood of $x_{0}$, so that $\varphi=u\left(x_{0}\right)$. In other words, $T_{x_{0}}(u)=\left\{u\left(x_{0}\right)\right\}$.

As a consequence, if on the contrary $\boldsymbol{\Xi}_{s}\left(u, x_{0}\right)>0$, then all tangent maps $\varphi \in T_{x_{0}}(u)$ must be non constant, and hence satisfy $\operatorname{dim} S(\varphi) \leqslant n-1$.

Lemma 7.12. Assume that $s \in[1 / 2,1)$. If $\varphi \in T_{x_{0}}(u)$ is not constant, then

$$
\operatorname{dim} S(\varphi) \leqslant n-2 .
$$

Proof. We proceed by contradiction assuming that there exists a non constant tangent map $\varphi \in T_{x_{0}}(u)$ such that $\operatorname{dim} S(\varphi)=n-1$. Rotating coordinates if necessary, we can assume that $S(\varphi)=\{0\} \times \mathbb{R}^{n-1}$. By Lemma 7.10, the map $\varphi$ only depends on the $x_{1}$-variable, that is $\varphi(x)=: \psi\left(x_{1}\right)$ where $\psi \in H_{\text {loc }}^{s}\left(\mathbb{R} ; \mathbb{S}^{d-1}\right)$. Since $\varphi$ is positively 0-homogeneous and non constant, the map $\psi$ is of the form

$$
\psi\left(x_{1}\right)=\left\{\begin{array}{ll}
\mathrm{a} & \text { if } x_{1}>0 \\
\mathrm{~b} & \text { if } x_{1}<0
\end{array},\right.
$$

for some points a, $\mathrm{b} \in \mathbb{S}^{d-1}, \mathrm{a} \neq \mathrm{b}$. However, a direct computation shows that $[\psi]_{H^{s}(-1,1)}=\infty$, i.e. $\psi \notin H^{s}(-1,1)$, a contradiction.

Lemma 7.13. Assume that $n \geqslant 2, s \in(0,1 / 2)$, and that $u$ is a minimizing s-harmonic map in $\Omega$. If $\varphi \in T_{x_{0}}(u)$ is not constant, then

$$
\operatorname{dim} S(\varphi) \leqslant n-2 .
$$

To prove Lemma 7.13, we shall make use of the following pleasant computation. 
Remark 7.14. For $n \geqslant 2$, we have

$$
\alpha_{n, s}:=\int_{\mathbb{R}^{n-1}} \frac{\mathrm{d} x^{\prime}}{\left(1+\left|x^{\prime}\right|^{2}\right)^{\frac{n+2 s}{2}}}=\frac{\gamma_{1, s}}{\gamma_{n, s}} .
$$

Indeed, we easily compute in polar coordinates and setting $t:=r^{2}$,

$$
\int_{\mathbb{R}^{n-1}} \frac{\mathrm{d} x^{\prime}}{\left(1+\left|x^{\prime}\right|^{2}\right)^{\frac{n+2 s}{2}}}=\left|\mathbb{S}^{n-2}\right| \int_{0}^{+\infty} \frac{r^{n-2}}{\left(1+r^{2}\right)^{\frac{n+2 s}{2}}} \mathrm{~d} r=\frac{\left|\mathbb{S}^{n-2}\right|}{2} \int_{0}^{+\infty} \frac{t^{\frac{n-1}{2}-1}}{(1+t)^{\frac{n+2 s}{2}}} \mathrm{~d} t .
$$

Recalling the value of $\gamma_{n, s}$ given in (2.1), we thus have

$$
\begin{aligned}
\int_{\mathbb{R}^{n-1}} \frac{\mathrm{d} x^{\prime}}{\left(1+\left|x^{\prime}\right|^{2}\right)^{\frac{n+2 s}{2}}}=\frac{\left|\mathbb{S}^{n-2}\right|}{2} \mathrm{~B}( & \left.\frac{n-1}{2}, \frac{1+2 s}{2}\right) \\
& =\frac{\left|\mathbb{S}^{n-2}\right|}{2} \frac{\Gamma\left(\frac{n-1}{2}\right) \Gamma\left(\frac{1+2 s}{2}\right)}{\Gamma\left(\frac{n+2 s}{2}\right)}=\pi^{\frac{n-1}{2}} \frac{\Gamma\left(\frac{1+2 s}{2}\right)}{\Gamma\left(\frac{n+2 s}{2}\right)}=\frac{\gamma_{1, s}}{\gamma_{n, s}}
\end{aligned}
$$

where $\mathrm{B}(\cdot, \cdot)$ denotes the Euler Beta function.

Proof of Lemma 7.13. Step 1. We proceed again by contradiction assuming that there exists a non constant tangent map $\varphi \in T_{x_{0}}(u)$ such that $\operatorname{dim} S(\varphi)=n-1$. Rotating coordinates if necessary, we can proceed as in the proof of Lemma 7.12 to infer that $\varphi(x)=: \psi\left(x_{1}\right)$ where $\psi \in H_{\text {loc }}^{s}\left(\mathbb{R} ; \mathbb{S}^{d-1}\right)$ is of the form $(7.13)$ for some points $\mathrm{a}, \mathrm{b} \in \mathbb{S}^{d-1}, \mathrm{a} \neq \mathrm{b}$. We claim that $\psi$ is a minimizing $s$-harmonic map in the interval $(-1,1)$. Once the claim is proved (which is the object of the next step), we can infer from the regularity result [34, Theorem 1.2] that $\psi$ is continuous in $(-1,1)$, which again enforces $\mathrm{a}=\mathrm{b}$, a contradiction.

Step 2. We now prove that $\psi$ is a minimizing $s$-harmonic map in $(-1,1)$. To this purpose, we fix an arbitrary competitor $v \in \widehat{H}^{s}\left((-1,1)\right.$; $\left.\mathbb{S}^{d-1}\right)$ such that $\operatorname{spt}(v-\psi) \subseteq(-1,1)$. Given $r>1$, we consider the open set $Q_{r} \subseteq \mathbb{R}^{n}$ defined by $Q_{r}:=(-1,1) \times D_{r}^{\prime}$ where $D_{r}^{\prime}$ denotes the open ball in $\mathbb{R}^{n-1}$ centered at the origin of radius $r$. We define a map $\widetilde{v}_{r} \in \widehat{H}^{s}\left(Q_{r} ; \mathbb{S}^{d-1}\right)$ by setting for $x=\left(x_{1}, x^{\prime}\right) \in \mathbb{R}^{n}$,

$$
\widetilde{v}_{r}(x):= \begin{cases}v\left(x_{1}\right) & \text { if }\left|x^{\prime}\right|<r \\ \psi\left(x_{1}\right) & \text { if }\left|x^{\prime}\right| \geqslant r .\end{cases}
$$

Recalling that $u$ is assumed to be minimizing, $\varphi$ is minimizing in every ball. Since $\operatorname{spt}\left(\widetilde{v}_{r}-\varphi\right) \subseteq$ $Q_{r+1}$, we thus have

$$
\mathcal{E}_{s}\left(\varphi, Q_{r+1}\right) \leqslant \mathcal{E}_{s}\left(\widetilde{v}_{r}, Q_{r+1}\right) .
$$

Since $\widetilde{v}_{r}=\varphi$ in $\mathbb{R}^{n} \backslash Q_{r}$, it reduces to

$$
\mathcal{E}_{s}\left(\varphi, Q_{r}\right) \leqslant \mathcal{E}_{s}\left(\widetilde{v}_{r}, Q_{r}\right)
$$

We claim that

$$
\frac{1}{\left|D_{r}^{\prime}\right|} \mathcal{E}_{s}\left(\widetilde{v}_{r}, Q_{r}\right) \underset{r \rightarrow \infty}{\longrightarrow} \mathcal{E}_{s}(v,(-1,1))
$$

where $\left|D_{r}^{\prime}\right|$ denotes the volume of $D_{r}^{\prime}$ in $\mathbb{R}^{n-1}$. Since we could have taken $v$ to be equal to $\psi$, (7.17) also holds with $\varphi$ in place of $\widetilde{v}_{r}$ and $\psi$ in place of $v$. Therefore, dividing both sides of (7.16) by $\left|D_{r}^{\prime}\right|$ and letting $r \rightarrow \infty$ leads to

$$
\mathcal{E}_{s}(\psi,(-1,1)) \leqslant \mathcal{E}_{s}(v,(-1,1)),
$$

which proves that $\psi$ is indeed minimizing in $(-1,1)$.

Let us now compute $\mathcal{E}_{s}\left(\widetilde{v}_{r}, Q_{r}\right)$ to prove (7.17). First, by Fubini's theorem we have

$$
\begin{aligned}
& \iint_{Q_{r} \times Q_{r}} \frac{\left|\widetilde{v}_{r}(x)-\widetilde{v}_{r}(y)\right|^{2}}{|x-y|^{n+2 s}} \mathrm{~d} x \mathrm{~d} y \\
& \quad=\iint_{(-1,1)^{2}}\left|v\left(x_{1}\right)-v\left(y_{1}\right)\right|^{2}\left(\iint_{D_{r}^{\prime} \times D_{r}^{\prime}} \frac{\mathrm{d} x^{\prime} \mathrm{d} y^{\prime}}{\left(\left|x_{1}-y_{1}\right|^{2}+\left|x^{\prime}-y^{\prime}\right|^{2}\right)^{\frac{n+2 s}{2}}}\right) \mathrm{d} x_{1} \mathrm{~d} y_{1} .
\end{aligned}
$$


Then we observe that a change of variables yields

$$
\begin{aligned}
\iint_{D_{r}^{\prime} \times D_{r}^{\prime}} \frac{\mathrm{d} x^{\prime} \mathrm{d} y^{\prime}}{\left(\left|x_{1}-y_{1}\right|^{2}+\left|x^{\prime}-y^{\prime}\right|^{2}\right)^{\frac{n+2 s}{2}}} & \\
=\iint_{D_{r}^{\prime} \times \mathbb{R}^{n-1}} \frac{\mathrm{d} x^{\prime} \mathrm{d} y^{\prime}}{\left(\left|x_{1}-y_{1}\right|^{2}+\left|x^{\prime}-y^{\prime}\right|^{2}\right)^{\frac{n+2 s}{2}}} & -A_{r}\left(\left|x_{1}-y_{1}\right|\right) \\
& =\frac{\alpha_{n, s}\left|D_{r}^{\prime}\right|}{\left|x_{1}-y_{1}\right|^{1+2 s}}-A_{r}\left(\left|x_{1}-y_{1}\right|\right),
\end{aligned}
$$

where $\alpha_{n, s}$ is given by $(7.14)$, and $A_{r}(t)$ is defined for $t>0$ by

$$
A_{r}(t):=\iint_{D_{r}^{\prime} \times\left(D_{r}^{\prime}\right)^{c}} \frac{\mathrm{d} x^{\prime} \mathrm{d} y^{\prime}}{\left(t^{2}+\left|x^{\prime}-y^{\prime}\right|^{2}\right)^{\frac{n+2 s}{2}}} .
$$

Therefore,

$$
\begin{aligned}
\iint_{Q_{r} \times Q_{r}} \frac{\left|\widetilde{v}_{r}(x)-\widetilde{v}_{r}(y)\right|^{2}}{|x-y|^{n+2 s}} \mathrm{~d} x \mathrm{~d} y= & \alpha_{n, s}\left|D_{r}^{\prime}\right| \iint_{(-1,1)^{2}} \frac{\left|v\left(x_{1}\right)-v\left(y_{1}\right)\right|^{2}}{\left|x_{1}-y_{1}\right|^{1+2 s}} \mathrm{~d} x_{1} \mathrm{~d} y_{1} \\
& -\iint_{(-1,1)^{2}}\left|v\left(x_{1}\right)-v\left(y_{1}\right)\right|^{2} A_{r}\left(\left|x_{1}-y_{1}\right|\right) \mathrm{d} x_{1} \mathrm{~d} y_{1} .
\end{aligned}
$$

Similarly, we compute

$$
\begin{aligned}
& \iint_{Q_{r} \times\left(Q_{r}\right)^{c}} \frac{\left|\widetilde{v}_{r}(x)-\widetilde{v}_{r}(y)\right|^{2}}{|x-y|^{n+2 s}} \mathrm{~d} x \mathrm{~d} y \\
& =\iint_{(-1,1) \times(-1,1)^{c}}\left|v\left(x_{1}\right)-v\left(y_{1}\right)\right|^{2}\left(\iint_{D_{r}^{\prime} \times \mathbb{R}^{n-1}} \frac{\mathrm{d} x^{\prime} \mathrm{d} y^{\prime}}{\left.\left(\left|x_{1}-y_{1}\right|^{2}+\left|x^{\prime}-y^{\prime}\right|^{2}\right)^{\frac{n+2 s}{2}}\right) \mathrm{d} x_{1} \mathrm{~d} y_{1}}\right. \\
& \quad+\iint_{(-1,1)^{2}}\left|v\left(x_{1}\right)-\psi\left(y_{1}\right)\right|^{2} A_{r}\left(\left|x_{1}-y_{1}\right|\right) \mathrm{d} x_{1} \mathrm{~d} y_{1},
\end{aligned}
$$

so that

$$
\begin{aligned}
\iint_{Q_{r} \times\left(Q_{r}\right)^{c}} \frac{\left|\widetilde{v}_{r}(x)-\widetilde{v}_{r}(y)\right|^{2}}{|x-y|^{n+2 s}} \mathrm{~d} x \mathrm{~d} y & =\alpha_{n, s}\left|D_{r}^{\prime}\right| \iint_{(-1,1) \times(-1,1)^{c}} \frac{\left|v\left(x_{1}\right)-v\left(y_{1}\right)\right|^{2}}{\left|x_{1}-y_{1}\right|^{1+2 s}} \mathrm{~d} x_{1} \mathrm{~d} y_{1} \\
& +\iint_{(-1,1)^{2}}\left|v\left(x_{1}\right)-\psi\left(y_{1}\right)\right|^{2} A_{r}\left(\left|x_{1}-y_{1}\right|\right) \mathrm{d} x_{1} \mathrm{~d} y_{1}
\end{aligned}
$$

Combining (7.18) and (7.19) leads to

$$
\frac{1}{\left|D_{r}^{\prime}\right|} \mathcal{E}_{s}\left(\widetilde{v}_{r}, Q_{r}\right)=\mathcal{E}_{s}(v,(-1,1))-I_{r}+I I_{r},
$$

where

$$
I_{r}:=\frac{\gamma_{1, s}}{4\left|D_{r}^{\prime}\right|} \iint_{(-1,1)^{2}}\left|v\left(x_{1}\right)-v\left(y_{1}\right)\right|^{2} A_{r}\left(\left|x_{1}-y_{1}\right|\right) \mathrm{d} x_{1} \mathrm{~d} y_{1},
$$

and

$$
I I_{r}:=\frac{\gamma_{1, s}}{2\left|D_{r}^{\prime}\right|} \iint_{(-1,1)^{2}}\left|v\left(x_{1}\right)-\psi\left(y_{1}\right)\right|^{2} A_{r}\left(\left|x_{1}-y_{1}\right|\right) \mathrm{d} x_{1} \mathrm{~d} y_{1} .
$$

Since $|v|=|\psi|=1$, we have

$$
I_{r}+I I_{r} \leqslant C r^{1-n} \iint_{(-1,1)^{2}} A_{r}\left(\left|x_{1}-y_{1}\right|\right) \mathrm{d} x_{1} \mathrm{~d} y_{1}
$$


and using Fubini's theorem again, we estimate

$$
\begin{aligned}
\iint_{(-1,1)^{2}} A_{r}\left(\left|x_{1}-y_{1}\right|\right) & \mathrm{d} x_{1} \mathrm{~d} y_{1} \\
& \leqslant \iint_{D_{r}^{\prime} \times\left(D_{r}^{\prime}\right)^{c}}\left(\iint_{(-1,1) \times \mathbb{R}} \frac{\mathrm{d} x_{1} \mathrm{~d} y_{1}}{\left(\left|x_{1}-y_{1}\right|^{2}+\left|x^{\prime}-y^{\prime}\right|^{2}\right)^{\frac{n+2 s}{2}}}\right) \mathrm{d} x^{\prime} \mathrm{d} y^{\prime} \\
& \leqslant C \iint_{D_{r}^{\prime} \times\left(D_{r}^{\prime}\right)^{c}} \frac{\mathrm{d} x^{\prime} \mathrm{d} y^{\prime}}{\left|x^{\prime}-y^{\prime}\right|^{n-1+2 s}} \\
& \leqslant C r^{n-1-2 s} .
\end{aligned}
$$

Therefore,

$$
\frac{1}{\left|D_{r}^{\prime}\right|} \mathcal{E}_{s}\left(\widetilde{v}_{r}, Q_{r}\right)=\mathcal{E}_{s}(v,(-1,1))+O\left(r^{-2 s}\right),
$$

and the proof is complete.

\subsection{Proof of Theorem 1.1, Theorem 1.2, and Theorem 1.3.}

Proof of Theorem 1.1. Let us fix an arbitrary point $x_{0} \in \Omega$, and set $r_{0}:=\frac{1}{2} \operatorname{dist}\left(x_{0}, \Omega^{c}\right)$. Without loss of generality, we can assume that $x_{0}=0$, so that our aim is to show that $u$ is smooth in a neighborhood of $x_{0}=0$. As noticed in Remark 4.3, the function $r \in\left(0,2 r_{0}-|\mathbf{x}|\right) \mapsto \boldsymbol{\Theta}_{s}\left(u^{\mathrm{e}}, \mathbf{x}, r\right)$ is nondecreasing for every $\mathbf{x} \in \partial^{0} B_{2 r_{0}}^{+}$. Moreover, since $2 s-n=2 s-1 \geqslant 0$, we have

$$
\lim _{r \rightarrow 0} \boldsymbol{\theta}_{s}(u, 0, r)=0 .
$$

Then we deduce from Corollary 2.20 that

$$
\lim _{r \rightarrow 0} \Theta_{s}\left(u^{\mathrm{e}}, 0, r\right)=0 .
$$

As a consequence, we can find $r_{1} \in\left(0, r_{0}\right)$ such that $\boldsymbol{\Theta}_{s}\left(u^{\mathrm{e}}, 0, r_{1}\right) \leqslant \varepsilon_{1}$, where the constant $\varepsilon_{1}$ is given by Corollary 4.2. From Theorem 5.1, we infer that $u \in C^{0,1}\left(D_{\kappa_{2}} r_{1}\right)$ for a constant $\kappa_{2} \in(0,1)$ depending only on $s$. In turn, Theorem 6.1 tells us that $u \in C^{\infty}\left(D_{\kappa_{2} r_{1} / 2}\right)$.

Proof of Theorem 1.2, case $s=1 / 2$. Considering the constant $\varepsilon_{1}>0$ given by Corollary 4.2 , we define

$$
\Sigma:=\left\{x \in \Omega: \boldsymbol{\Xi}_{s}(u, x) \geqslant \varepsilon_{1}\right\}
$$

By Corollary $2.18, \Sigma$ is relatively closed subset of $\Omega$. On the other hand, it is well known that $\mathcal{H}^{n-1}(\Sigma)=0$, see e.g. [58, Corollary 3.2.3].

We claim that $u \in C^{\infty}(\Omega \backslash \Sigma)$. Indeed, if $x_{0} \in \Omega \backslash \Sigma$, then we can find a radius $r \in$ $\left(0, \frac{1}{2} \operatorname{dist}\left(x_{0}, \Omega^{c}\right)\right)$ such that $\Theta_{s}\left(u^{\mathrm{e}}, 0, r\right) \leqslant \varepsilon_{1}$. Applying Theorem 5.1 and Theorem 6.1, we conclude that $u \in C^{\infty}\left(D_{\kappa_{2} r / 2}\right)$, and the claim is proved.

Obviously, $\operatorname{sing}(u) \subseteq \Sigma$, and it now only remains to show that $\operatorname{sing}(u)=\Sigma$. This is in fact a direct consequence of the regularity result in [21, Theorem 4.1]. Indeed, assume by contradiction that there is a point $x_{0} \in \Sigma \backslash \operatorname{sing}(u)$. Since $\operatorname{sing}(u)$ is a relatively closed subset of $\Omega$, we can find $r>0$ such that $D_{2 r}\left(x_{0}\right) \subseteq \Omega \backslash \operatorname{sing}(u)$, i.e., $u$ is continuous in $D_{2 r}\left(x_{0}\right)$. Consequently, $u^{\mathrm{e}}$ is continuous in $B_{r}^{+}\left(\mathbf{x}_{0}\right) \cup \partial^{0} B_{r}^{+}\left(\mathbf{x}_{0}\right)$, where $\mathbf{x}_{0}=\left(x_{0}, 0\right)$. However, by Proposition 3.13 (with $s=1 / 2), u^{\mathrm{e}} \in H^{1}\left(B_{r}^{+}\left(\mathbf{x}_{0}\right) ; \mathbb{R}^{d}\right)$ also solves

$$
\int_{B_{r}^{+}\left(x_{0}\right)} \nabla u^{\mathrm{e}} \cdot \nabla \Phi \mathrm{d} \mathbf{x}=0
$$

for every $\Phi \in H^{1}\left(B_{r}^{+}\left(\mathbf{x}_{0}\right) ; \mathbb{R}^{d}\right)$ such that $\Phi=0$ on $\partial^{+} B_{r}\left(\mathbf{x}_{0}\right)$ and $u \cdot \Phi=0$ on $\partial^{0} B_{r}\left(\mathbf{x}_{0}\right)$. Then [21, Theorem 4.1] tells us that $u^{\mathrm{e}} \in C^{1, \alpha}\left(B_{r / 2}^{+}\left(x_{0}\right)\right)$ for every $\alpha \in(0,1)$. Consequently, $\Xi_{s}\left(u, x_{0}\right)=0$, i.e., $x_{0} \notin \Sigma$, a contradiction.

Proof of Theorem 1.2, case $s \neq 1 / 2$. We still consider the relatively closed subset $\Sigma$ of $\Omega$ defined in (7.20). As in the case $s=1 / 2$, it follows from Theorem 5.1 and Theorem 6.1 that $u \in$ $C^{\infty}(\Omega \backslash \Sigma)$. In particular, $\operatorname{sing}(u) \subseteq \Sigma$. On the other hand, if $u$ is continuous in a neighborhood 
of a point $x_{0} \in \Omega$, then $T_{x_{0}}(u)=\left\{u\left(x_{0}\right)\right\}$, and thus $\boldsymbol{\Xi}_{s}\left(u, x_{0}\right)=0$. Hence, $x_{0} \notin \Sigma$, and we conclude that $\operatorname{sing}(u)=\Sigma$. In view of Remark 7.11 and Lemma 7.12, we have

$$
\Sigma= \begin{cases}\left\{x \in \Omega: \operatorname{dim} S(\varphi) \leqslant n-1 \forall \varphi \in T_{x}(u)\right\} & \text { if } s \in(0,1 / 2) ; \\ \left\{x \in \Omega: \operatorname{dim} S(\varphi) \leqslant n-2 \forall \varphi \in T_{x}(u)\right\} & \text { if } s \in(1 / 2,1) .\end{cases}
$$

We can now apply e.g. [53, Chapter 3.4, proof of Lemma 1] (which only relies on the upper semicontinuity of $\boldsymbol{\Xi}_{s}$ stated in Corollary 7.7, the strong convergence of blow-ups to tangent maps, and the structure results on tangent maps established in Subection 7.2) to conclude that $\operatorname{dim}_{\mathcal{H}} \Sigma \leqslant n-1$ for $s \in(0,1 / 2), \operatorname{dim}_{\mathcal{H}} \Sigma \leqslant n-2$ for $s \in(1 / 2,1)$, and that $\Sigma$ is locally finite in $\Omega$ if $n=1$ with $s \in(0,1 / 2)$ or $n=2$ with $s \in(1 / 2,1)$.

Proof of Theorem 1.3. For $s \in(1 / 2,1)$, we simply apply Theorem 1.2 (recalling that minimality implies stationarity). We thus assume that $s \in(0,1 / 2]$. Since $u$ is minimizing in $\Omega$, the results in Subsection 7.2 apply. Hence, we can repeat the proof of Theorem 1.2 to derive that $u \in C^{\infty}(\Omega \backslash \Sigma), \operatorname{sing}(u)=\Sigma$, where $\Sigma$ is still given by (7.20). In view of Lemma 7.12 and Lemma 7.13, we now have

$$
\Sigma=\left\{x \in \Omega: \operatorname{dim} S(\varphi) \leqslant n-2 \forall \varphi \in T_{x}(u)\right\} .
$$

Once again, [53, Chapter 3.4, proof of Lemma 1] shows that $\operatorname{dim}_{\mathcal{H}} \Sigma \leqslant n-2$, and that $\Sigma$ is locally finite in $\Omega$ if $n=2$.

We complete this section with an example showing that Theorem 1.3 is sharp in every space dimension for $s=1 / 2$.

Example 7.15. In [30, Theorem 1.4], it has been proved that the map $u_{2 \mathrm{D}}: \mathbb{R}^{2} \rightarrow \mathbb{S}^{1}$ given by

$$
u_{2 \mathrm{D}}(x):=\frac{x}{|x|},
$$

is a (0-homogeneous) minimizing 1/2-harmonic map. Obviously, the origin belongs to $\operatorname{sing}\left(u_{2 \mathrm{D}}\right)$.

In arbitrary dimension $n>2$, we consider the 0-homogeneous map $u_{\star}: \mathbb{R}^{n} \rightarrow \mathbb{S}^{1}$ defined as

$$
u_{\star}(x):=u_{2 \mathrm{D}}\left(x^{\prime}\right),
$$

with $x^{\prime}:=\left(x_{1}, x_{2}\right) \in \mathbb{R}^{2}$ and $x=\left(x^{\prime}, x^{\prime \prime}\right)$. Then $u_{\star}$ is a minimizing $1 / 2$-harmonic map into $\mathbb{S}^{1}$ with $\operatorname{sing}\left(u_{\star}\right)=\{(0,0)\} \times \mathbb{R}^{n-2}$.

To prove that $u_{\star}$ is minimizing, we proceed as follows. First notice that $u_{\star} \in \widehat{H}^{1 / 2}\left(D_{R} ; \mathbb{R}^{2}\right) \cap$ $H_{\text {loc }}^{1 / 2}\left(\mathbb{R}^{n}\right)$ for every $R>0$. According to [30, Definition 3.1 and Theorem 3.6], it is enough to show that $u_{\star}^{\mathrm{e}}$ is a minimizing harmonic map with (partially) free boundary in every admissible bounded open set $G \subseteq \mathbb{R}_{+}^{n+1}$. In turn, by 0-homogeneity of $u_{\star}^{\mathrm{e}}$, it is enough to show that $u_{\star}^{\mathrm{e}}$ is a minimizing harmonic map with (partially) free boundary in a given admissible bounded open set $G \subseteq \mathbb{R}_{+}^{n+1}$, that we choose to be

$$
G:=D_{1}^{(2)} \times(-1,1)^{n-2} \times(0,1),
$$

where $D_{1}^{(2)}$ denotes the open unit disc in $\mathbb{R}^{2}$ centered at the origin. Applying Fubini's theorem and changing variables, we start computing for $z>0$,

$$
\begin{aligned}
u_{\star}^{\mathrm{e}}(x, z) & =\gamma_{n, \frac{1}{2}} \int_{\mathbb{R}^{2}} z u_{2 \mathrm{D}}\left(y^{\prime}\right)\left(\int_{\mathbb{R}^{n-2}} \frac{\mathrm{d} y^{\prime \prime}}{\left(\left|x^{\prime}-y^{\prime}\right|^{2}+\left|x^{\prime \prime}-y^{\prime \prime}\right|^{2}+z^{2}\right)^{\frac{n+1}{2}}}\right) \mathrm{d} y^{\prime} \\
& =\gamma_{n, \frac{1}{2}}\left(\int_{\mathbb{R}^{n-2}} \frac{\mathrm{d} y^{\prime \prime}}{\left(\left|y^{\prime \prime}\right|^{2}+1\right)^{\frac{n+1}{2}}}\right) \int_{\mathbb{R}^{2}} \frac{z u_{2 \mathrm{D}}\left(y^{\prime}\right)}{\left(\left|x^{\prime}-y^{\prime}\right|^{2}+z^{2}\right)^{\frac{3}{2}}} \mathrm{~d} y^{\prime},
\end{aligned}
$$

where we have used that $\sigma_{n, \frac{1}{2}}=\gamma_{n, \frac{1}{2}}$. As in Remark 7.14, we have

Hence,

$$
\int_{\mathbb{R}^{n-2}} \frac{\mathrm{d} y^{\prime \prime}}{\left(\left|y^{\prime \prime}\right|^{2}+1\right)^{\frac{n+1}{2}}}=\pi^{\frac{n-2}{2}} \frac{\Gamma(3 / 2)}{\Gamma\left(\frac{n+1}{2}\right)}=\frac{\gamma_{2, \frac{1}{2}}}{\gamma_{n, \frac{1}{2}}} .
$$

$$
u_{\star}^{\mathrm{e}}(x, z)=u_{2 \mathrm{D}}^{\mathrm{e}}\left(x^{\prime}, z\right) .
$$

Now we consider a competitor $v \in H^{1}\left(G ; \mathbb{R}^{2}\right)$ satisfying $v(\mathbf{x}) \in \mathbb{S}^{1}$ for a.e. $\mathbf{x} \in \partial^{0} G$ and $\operatorname{spt}\left(v-u_{\star}^{\mathrm{e}}\right) \subseteq G \cup \partial^{0} G$. By a classical slicing property for Sobolev functions, for a.e. $x^{\prime \prime} \in$ 
$(-1,1)^{n-2}$, we have: (i) $v\left(\cdot, x^{\prime \prime}, \cdot\right) \in H^{1}\left(D_{1}^{(2)} \times(0,1)\right.$; $\left.\mathbb{R}^{2}\right)$; (ii) $v\left(x^{\prime}, x^{\prime \prime}, 0\right) \in \mathbb{S}^{1}$ for a.e. $x^{\prime} \in D_{1}^{(2)}$; (iii) $\operatorname{spt}\left(v\left(\cdot, x^{\prime \prime}, \cdot\right)-u_{2 \mathrm{D}}\right) \subseteq D_{1}^{(2)} \times[0,1)$. By minimality of $u_{2 \mathrm{D}}$ and [30, Theorem 3.6], we infer that

$\int_{D_{1}^{(2)} \times(0,1)}\left|\nabla_{x^{\prime}, z} v\left(x^{\prime}, x^{\prime \prime}, z\right)\right|^{2} \mathrm{~d} x^{\prime} \mathrm{d} z \geqslant \int_{D_{1}^{(2)} \times(0,1)}\left|\nabla u_{2 \mathrm{D}}^{\mathrm{e}}\left(x^{\prime}, z\right)\right|^{2} \mathrm{~d} x^{\prime} \mathrm{d} z$ for a.e. $x^{\prime \prime} \in(-1,1)^{n-2}$.

It now follows from Fubini's theorem and (7.21) that

$$
\begin{aligned}
\mathbf{E}_{\frac{1}{2}}(v, G) \geqslant \frac{1}{2} \int_{(-1,1)^{n-2}} & \left(\int_{D_{1}^{(2)} \times(0,1)}\left|\nabla_{x^{\prime}, z} v\left(x^{\prime}, x^{\prime \prime}, z\right)\right|^{2} \mathrm{~d} x^{\prime} \mathrm{d} z\right) \mathrm{d} x^{\prime \prime} \\
\geqslant & \frac{1}{2} \int_{(-1,1)^{n-2}}\left(\int_{D_{1}^{(2)} \times(0,1)}\left|\nabla u_{2 \mathrm{D}}^{\mathrm{e}}\left(x^{\prime}, z\right)\right|^{2} \mathrm{~d} x^{\prime} \mathrm{d} z\right) \mathrm{d} x^{\prime \prime}=\mathbf{E}_{\frac{1}{2}}\left(u_{\star}^{\mathrm{e}}, G\right),
\end{aligned}
$$

and the minimality of $u_{\star}^{\mathrm{e}}$ is proved.

\section{Appendix A. On the Degenerate Laplace equation}

In this first appendix, our aim is to recall some of the properties satisfied by weak solutions of the (scalar) degenerate linear elliptic equation

$$
\operatorname{div}\left(|z|^{a} \nabla w\right)=0 \quad \text { in } B_{R}\left(\mathbf{x}_{\mathbf{0}}\right),
$$

with $\mathbf{x}_{0}=\left(x_{0}, z_{0}\right) \in \mathbb{R}^{n+1}$. Those properties are essentially taken from [40], and we reproduce here the statements for convenience of the reader. The notion of weak solution to this equation corresponds to the variational formulation. In other words, we say that $w \in H^{1}\left(B_{R}\left(\mathbf{x}_{0}\right),|z|^{a} \mathrm{~d} \mathbf{x}\right)$ is a weak solution of (A.1) if

$$
\int_{B_{R}\left(\mathbf{x}_{0}\right)}|z|^{a} \nabla w \cdot \nabla \Phi \mathrm{d} \mathbf{x}=0
$$

for every $\Phi \in H^{1}\left(B_{R}\left(\mathbf{x}_{0}\right),|z|^{a} \mathrm{dx}\right)$ such that $\Phi=0$ on $\partial B_{R}\left(\mathbf{x}_{\mathbf{0}}\right)$.

One may complement (A.1) with a boundary condition of the form $w=v$ on $\partial B_{R}\left(\mathbf{x}_{0}\right)$ for a given $v \in H^{1}\left(B_{R}\left(\mathbf{x}_{0}\right),|z|^{a} \mathrm{~d} \mathbf{x}\right)$. This boundary condition is thus interpreted in the sense of traces. Classically, such a boundary condition uniquely determines the solution of (A.1) which can be characterized by energy minimality.

Lemma A.1. Let $v \in H^{1}\left(B_{R}\left(\mathbf{x}_{0}\right),|z|^{a} \mathrm{~d} \mathbf{x}\right)$. The equation

$$
\begin{cases}\operatorname{div}\left(|z|^{a} \nabla w\right)=0 & \text { in } B_{R}\left(\mathbf{x}_{\mathbf{0}}\right), \\ w=v & \text { on } \partial B_{R}\left(\mathbf{x}_{0}\right),\end{cases}
$$

admits a unique weak solution which is characterized by

$$
\int_{B_{R}\left(\mathbf{x}_{0}\right)}|z|^{a}|\nabla w|^{2} \mathrm{~d} \mathbf{x} \leqslant \int_{B_{R}\left(\mathbf{x}_{0}\right)}|z|^{a}|\nabla \Phi|^{2} \mathrm{~d} \mathbf{x}
$$

for every $\Phi \in H^{1}\left(B_{R}\left(\mathbf{x}_{0}\right),|z|^{a} \mathrm{~d} \mathbf{x}\right)$ satisfying $\Phi=v$ on $\partial B_{R}\left(\mathbf{x}_{\mathbf{0}}\right)$.

As for the usual Laplace equation, energy minimality can be used to prove that $w$ inherits symmetries from the boundary condition. In our case, we make use of the following lemma.

Lemma A.2. Let $\mathbf{x}_{0} \in \mathbb{R}^{n} \times\{0\}$ and $v \in H^{1}\left(B_{R},|z|^{a} \mathrm{~d} \mathbf{x}\right)$. If $v$ is symmetric with respect to $\{z=0\}$, then the weak solution $w$ of (A.2) is also symmetric with respect to $\{z=0\}$.

Concerning interior regularity of weak solutions, the issue is of course near the hyperplane $\{z=0\}$. Indeed, if the ball $B_{R}\left(\mathbf{x}_{0}\right)$ is away from $\{z=0\}$, then the operator becomes uniformly elliptic with smooth coefficients, and the classical elliptic theory tells us that weak solutions are $C^{\infty}$ in the interior. For an arbitrary ball, the general results of [18] about degenerate elliptic equations apply, and they provide at least local Hölder continuity in the interior. Using the invariance of the equation with respect to the $x$-variables, the regularity can be further improved (see e.g. [40, Corollary 2.13]). Some boundary regularity and related maximum principles are also known from the general theory in [23]. We reproduce here the statement in [40, Lemma 2.18]. 
Lemma A.3. Let $v \in H^{1}\left(B_{R}\left(\mathbf{x}_{0}\right),|z|^{a} \mathrm{~d} \mathbf{x}\right) \cap C^{0}\left(\bar{B}_{R}\left(\mathbf{x}_{0}\right)\right)$. The weak solution $w$ of (A.2) belongs to $C^{0}\left(\bar{B}_{R}\left(\mathbf{x}_{0}\right)\right)$. Moreover,

$$
\min _{\bar{B}_{R}\left(\mathbf{x}_{0}\right)} w=\min _{\partial B_{R}\left(\mathbf{x}_{0}\right)} v \text { and } \max _{\bar{B}_{R}\left(\mathbf{x}_{0}\right)} w=\max _{\partial B_{R}\left(\mathbf{x}_{0}\right)} v .
$$

A further fundamental property of weak solutions of (A.1) is an energy monotonicity in which one has to distinguish balls centered at a point of $\{z=0\}$ from balls lying away from $\{z=0\}$. The two following lemmas are taken from [40, Lemma 2.8] and [40, Lemma 2.17], respectively.

Lemma A.4. Let $\mathbf{x}_{0} \in \mathbb{R}^{n} \times\{0\}$ and $w \in H^{1}\left(B_{R}\left(\mathbf{x}_{0}\right),|z|^{a} \mathrm{~d} \mathbf{x}\right)$ a weak solution of (A.1). Assume that either $s \geqslant 1 / 2$, or that $s<1 / 2$ and $w$ is symmetric with respect to the hyperplane $\{z=0\}$. Then,

for every $0<\rho \leqslant r \leqslant R$

$$
\frac{1}{\rho^{n+2-2 s}} \int_{B_{\rho}\left(\mathbf{x}_{0}\right)}|z|^{a}|\nabla w|^{2} \mathrm{~d} \mathbf{x} \leqslant \frac{1}{r^{n+2-2 s}} \int_{B_{r}\left(\mathbf{x}_{0}\right)}|z|^{a}|\nabla w|^{2} \mathrm{~d} \mathbf{x}
$$

Lemma A.5. Let $w \in H^{1}\left(B_{R}\left(\mathbf{x}_{0}\right),|z|^{a} \mathrm{~d} \mathbf{x}\right)$ be a weak solution of $(\mathrm{A} .1)$. If $\mathbf{x}_{0}=\left(x_{0}, z_{0}\right) \in \mathbb{R}_{+}^{n+1}$ and $R>0$ are such that $B_{R}\left(\mathbf{x}_{0}\right) \subseteq \mathbb{R}_{+}^{n+1}$ and $z_{0} \geqslant \theta R$ for some $\theta \geqslant 2$, then

$$
\left(\frac{2}{R}\right)^{n+1} \int_{B_{R / 2}\left(\mathbf{x}_{0}\right)}|z|^{a}|\nabla w|^{2} \mathrm{~d} \mathbf{x} \leqslant\left(1+\frac{C}{\theta-1}\right) \frac{1}{R^{n+1}} \int_{B_{R}\left(\mathbf{x}_{0}\right)}|z|^{a}|\nabla w|^{2} \mathrm{~d} \mathbf{x},
$$

for a constant $C=C(n)$.

\section{ApPendix B. A Lipschitz ESTIMATE FOR $s$-HARMONIC FUnCTIONS}

The purpose of this appendix is to provide an interior Lipschitz estimate for weak solutions $w \in \widehat{H}^{s}\left(D_{1}\right)$ of the fractional Laplace equation

$$
(-\Delta)^{s} w=0 \quad \text { in } H^{-s}\left(D_{1}\right) .
$$

The notion of weak solution is understood here according to the weak formulation of the $s$ Laplacian operator, see (2.3). Interior regularity for weak solutions is known, and it tells us that $w$ is locally $C^{\infty}$ in $D_{1}$. The following estimate is probably also well known, but we give a proof for convenience of the reader.

Lemma B.1. If $w \in \widehat{H}^{s}\left(D_{1}\right)$ is a weak solution of (B.1), then $w \in C^{\infty}\left(D_{1 / 2}\right)$, and

$$
\|w\|_{L^{\infty}\left(D_{1 / 2}\right)}^{2}+\|\nabla w\|_{L^{\infty}\left(D_{1 / 2}\right)}^{2} \leqslant C\left(\mathcal{E}_{s}\left(w, D_{1}\right)+\|w\|_{L^{2}\left(D_{1}\right)}^{2}\right),
$$

for a constant $C=C(n, s)$.

Proof. As we already mentioned, the regularity theory is already known, and we take advantage of this to only derive estimate (B.2). Let us fix an arbitrary point $x_{0} \in D_{1 / 2}$. We consider the extension $w^{\mathrm{e}}$ which belongs to $H^{1}\left(B_{1 / 4}^{+}\left(\mathbf{x}_{0}\right),|z|^{a} \mathrm{~d} \mathbf{x}\right)$ with $\mathbf{x}_{0}:=\left(x_{0}, 0\right)$ by Lemma 2.9. In view of Lemma 2.12, it satisfies

$$
\int_{B_{1 / 4}^{+}\left(\mathbf{x}_{0}\right)} z^{a} \nabla w^{\mathrm{e}} \cdot \nabla \Phi \mathrm{d} x=0
$$

for every $\Phi \in H^{1}\left(B_{1 / 4}^{+}\left(\mathbf{x}_{0}\right),|z|^{a} \mathrm{~d} \mathbf{x}\right)$ such that $\Phi=0$ on $\partial^{+} B_{1 / 4}^{+}\left(\mathbf{x}_{0}\right)$. Then we consider the even extension of $w^{\mathrm{e}}$ to the whole ball $B_{1 / 4}\left(\mathbf{x}_{0}\right)$ that we still denote by $w^{\mathrm{e}}$ (i.e. $w^{\mathrm{e}}(x, z)=w^{\mathrm{e}}(x,-z)$ ). Then $w^{\mathrm{e}} \in H^{1}\left(B_{1 / 4}\left(\mathbf{x}_{0}\right),|z|^{a} \mathrm{~d} \mathbf{x}\right)$, and arguing as in the proof of Corollary 5.4, we infer that $w^{\mathrm{e}}$ is a weak solution of (A.1) with $R=1 / 4$. According to [40, Corollary 2.13], the weak derivatives $\partial_{i} w^{\mathrm{e}}$ belongs to $H^{1}\left(B_{1 / 8}\left(\mathbf{x}_{0}\right),|z|^{a} \mathrm{~d} \mathbf{x}\right)$ for $i=1, \ldots, n$, and they are weak solutions of (A.1) with $R=1 / 8$. Now, applying [18, Theorem 2.3.12] to $w^{\mathrm{e}}$ and $\partial_{i} w^{\mathrm{e}}$, we infer that $w^{\mathrm{e}} \in C^{1, \alpha}\left(B_{1 / 16}\left(\mathbf{x}_{0}\right)\right)$ for some exponent $\alpha=\alpha(n, s) \in(0,1)$,

$$
\left[w^{\mathrm{e}}\right]_{C^{0, \alpha}\left(B_{1 / 16}\left(\mathbf{x}_{0}\right)\right)} \leqslant C\left\|w^{\mathrm{e}}\right\|_{L^{2}\left(B_{1 / 8}\left(\mathbf{x}_{0}\right),|z|^{a} \mathrm{~d} \mathbf{x}\right)},
$$

and

$$
\left[\nabla_{x} w^{\mathrm{e}}\right]_{C^{0, \alpha}\left(B_{1 / 16}\left(\mathbf{x}_{0}\right)\right)} \leqslant C\left\|\nabla_{x} w^{\mathrm{e}}\right\|_{L^{2}\left(B_{1 / 8}\left(\mathbf{x}_{0}\right),|z|^{a} \mathrm{~d} \mathbf{x}\right)},
$$

for a constant $C=C(n, s)$. 
On the other hand, for every $\mathbf{x} \in B_{1 / 16}\left(\mathbf{x}_{0}\right)$, we have (recall our notation in (5.11))

$$
\begin{array}{r}
\left|w^{\mathrm{e}}(\mathbf{x})\right| \leqslant\left.\left.\left|w^{\mathrm{e}}(\mathbf{x})-\frac{1}{\left|B_{1 / 16}\right|_{a}} \int_{B_{1 / 16}\left(x_{0}\right)}\right| z\right|^{a} w^{\mathrm{e}}(\mathbf{y}) \mathrm{d} \mathbf{y}\left|+\frac{1}{\left|B_{1 / 16}\right|_{a}} \int_{B_{1 / 16}\left(x_{0}\right)}\right| z\right|^{a}\left|w^{\mathrm{e}}(\mathbf{y})\right| \mathrm{d} \mathbf{y} \\
\leqslant C\left(\left[w^{\mathrm{e}}\right]_{C^{0, \alpha}\left(B_{1 / 16}\left(\mathbf{x}_{0}\right)\right)}+\left\|w^{\mathrm{e}}\right\|_{L^{2}\left(B_{1 / 16}\left(\mathbf{x}_{0}\right),|z|^{a} \mathrm{~d} \mathbf{x}\right)}\right) .
\end{array}
$$

Combining this estimate with (B.3) and Lemma 2.9 leads to

$$
\left\|w^{\mathrm{e}}\right\|_{L^{\infty}\left(B_{1 / 16}\left(x_{0}\right)\right)}^{2} \leqslant C\left(\mathcal{E}_{s}\left(w, D_{1}\right)+\|w\|_{L^{2}\left(D_{1}\right)}^{2}\right) .
$$

The same argument applied to $\nabla_{x} w^{\mathrm{e}}$ and using (B.4) instead of (B.3) yields

$$
\left\|\nabla_{x} w^{\mathrm{e}}\right\|_{L^{\infty}\left(B_{1 / 16}\left(x_{0}\right)\right)}^{2} \leqslant C\left\|\nabla_{x} w^{\mathrm{e}}\right\|_{L^{2}\left(B_{1 / 8}\left(\mathbf{x}_{0}\right),|z|^{a} \mathrm{~d} \mathbf{x}\right)}^{2} \leqslant C \mathcal{E}_{s}\left(w, D_{1}\right),
$$

thanks to Lemma 2.9 again. Now the conclusion follows from the fact that $w^{\mathrm{e}}=w$ and $\nabla_{x} w^{\mathrm{e}}=\nabla w$ on $\partial^{0} B_{1 / 16}^{+}\left(\mathbf{x}_{0}\right)$.

\section{Appendix C. An embedding theorem Between generalized $\mathcal{Q}_{\alpha}$-SPACES}

In this appendix, our goal is to prove one of the crucial estimates used in the proof of Theorem 4.1, Corollary C.6 below. In turns out that this estimate does not explicitly appear in the existing literature (to the best of our knowledge), but it can be shortly derived from recent results in harmonic analysis. The purpose of this appendix is thus to explain how to combine those results to reach our goal. First, we need to recall some definitions and notations.

The space $\mathscr{S}_{\infty}\left(\mathbb{R}^{n}\right)$ can be defined as the topological subspace of the Schwartz class $\mathscr{S}\left(\mathbb{R}^{n}\right)$ made of all functions $\varphi$ such that the semi-norm

$$
\|\varphi\|_{M}:=\sup _{|\gamma| \leqslant M} \sup _{\xi \in \mathbb{R}^{n}}\left|\partial^{\gamma} \widehat{\varphi}(\xi)\right|\left(|\xi|^{M}+|\xi|^{-M}\right)
$$

is finite for every $M \in \mathbb{N}$, where $\gamma=\left(\gamma_{1}, \ldots, \gamma_{n}\right) \in \mathbb{N}^{n},|\gamma|:=\gamma_{1}+\ldots+\gamma_{n}$, and $\partial^{\gamma}:=\partial_{1}^{\gamma_{1}} \ldots \partial_{n}^{\gamma_{n}}$. Its topological dual is denoted by $\mathscr{S}_{\infty}^{\prime}\left(\mathbb{R}^{n}\right)$, and it is endowed with the weak $*$-topology, see e.g. $[55,57]$.

The following $\mathcal{Q}_{p}^{\alpha, q}$-spaces were introduced in [5, 57], generalizing the notion of $\mathcal{Q}_{\alpha}$-space (see [51, Section 1.2.4] and references therein), in the sense that $\mathcal{Q}_{\alpha}\left(\mathbb{R}^{n}\right)=\mathcal{Q}_{n / \alpha}^{\alpha, 2}\left(\mathbb{R}^{n}\right)$.

Definition C.1 ([5, 57]). Given $\alpha \in(0,1), p \in(0, \infty]$ and $q \in[1, \infty)$, define $\mathcal{Q}_{p}^{\alpha, q}\left(\mathbb{R}^{n}\right)$ as the space made of elements $f \in \mathscr{S}_{\infty}^{\prime}\left(\mathbb{R}^{n}\right)$ such that $f(x)-f(y)$ is a measurable function on $\mathbb{R}^{n} \times \mathbb{R}^{n}$ and

$$
\|f\|_{\mathcal{Q}_{p}^{\alpha, q}\left(\mathbb{R}^{n}\right)}:=\sup _{Q}|Q|^{\frac{1}{p}-\frac{1}{q}}\left(\iint_{Q \times Q} \frac{|f(x)-f(y)|^{q}}{|x-y|^{n+\alpha q}} \mathrm{~d} x \mathrm{~d} y\right)^{1 / q}<+\infty,
$$

where $Q$ ranges over all cubes of dyadic edge lengths in $\mathbb{R}^{n}$.

Remark C.2. Endowed with $\|\cdot\|_{\mathcal{Q}_{p}^{\alpha, q}\left(\mathbb{R}^{n}\right)}$, the space $\mathcal{Q}_{p}^{\alpha, q}\left(\mathbb{R}^{n}\right)$ is a semi-normed vector space, and

$$
N_{\alpha, p, q}(f):=\sup _{D_{r}\left(x_{0}\right) \subseteq \mathbb{R}^{n}} r^{\frac{n}{p}-\frac{n}{q}}\left(\iint_{D_{r}\left(x_{0}\right) \times D_{r}\left(x_{0}\right)} \frac{|f(x)-f(y)|^{q}}{|x-y|^{n+\alpha q}} \mathrm{~d} x \mathrm{~d} y\right)^{1 / q}
$$

provides an equivalent semi-norm.

The following embeddings between $\mathcal{Q}_{p}^{\alpha, q}$-spaces hold.

Theorem C.3. If $0<\alpha_{1}<\alpha_{2}<1,1 \leqslant q_{2}<q_{1}<\infty$, and $0<\lambda \leqslant n$ are such that

$$
\alpha_{1}-\frac{\lambda}{q_{1}}=\alpha_{2}-\frac{\lambda}{q_{2}}
$$

then $\mathcal{Q}_{\frac{n q_{2}}{\lambda}}^{\alpha_{2}, q_{2}}\left(\mathbb{R}^{n}\right) \hookrightarrow \mathcal{Q}_{\frac{n q_{1}}{\lambda}}^{\alpha_{1}, q_{1}}\left(\mathbb{R}^{n}\right)$ continuously.

As we briefly mentioned at the beginning of this appendix, this theorem actually follows quite directly from a more general embedding result between some homogeneous Triebel-LizorkinMorrey-Lorentz spaces [26] together with an identification result between various definitions of homogeneous Triebel-Lizorkin-Morrey type spaces [42], and a characterization of the $\mathcal{Q}_{p}^{\alpha, q_{-}}$ spaces within this scale of spaces [57]. We refer to the monograph [51] for what concerns the 
spaces involved here, and we limit ourselves to their basic definition. To this purpose, we consider a reference bump function $\psi \in \mathscr{S}\left(\mathbb{R}^{n}\right)$ such that

$$
\operatorname{spt} \widehat{\psi} \subseteq\left\{\xi \in \mathbb{R}^{n}: \frac{1}{2} \leqslant|\xi| \leqslant 2\right\} \quad \text { and }|\widehat{\psi}(\xi)| \geqslant C>0 \text { for } \frac{3}{5} \leqslant|\xi| \leqslant \frac{5}{3} .
$$

(In particular, $\psi \in \mathscr{S}_{\infty}\left(\mathbb{R}^{n}\right)$.) For $j \in \mathbb{Z}$, we denote by $\psi_{j}$ the function defined by

$$
\psi_{j}(x):=2^{j n} \psi\left(2^{j} x\right) .
$$

Definition C.4. Given $p, q \in(0, \infty), s \in \mathbb{R}$, and $\tau \in[0, \infty)$, the homogeneous Triebel-Lizorkin space $\dot{F}_{p, q}^{s, \tau}\left(\mathbb{R}^{n}\right)$ is defined to be the set of all $f \in \mathscr{S}_{\infty}^{\prime}\left(\mathbb{R}^{n}\right)$ such that

$$
\|f\|_{\dot{F}_{p, q}^{s, \tau}\left(\mathbb{R}^{n}\right)}:=\sup _{Q} \frac{1}{|Q|^{\tau}}\left(\int_{Q}\left(\sum_{j=j_{Q}}^{\infty}\left(2^{j s}\left|\psi_{j} * f(x)\right|\right)^{q}\right)^{p / q} \mathrm{~d} x\right)^{1 / p}<+\infty,
$$

where $Q$ ranges over all cubes of dyadic edge lengths in $\mathbb{R}^{n}$, and $j_{Q}:=-\log _{2} \ell(Q)$ with $\ell(Q)$ the edge length of $Q$.

Definition C.5. Given $0<p \leqslant u<\infty, 0<q<\infty$, and $s \in \mathbb{R}$, the homogeneous TriebelLizorkin-Morrey space $\dot{\mathcal{E}}_{p, q, u}^{s}\left(\mathbb{R}^{n}\right)$ is defined to be the set of all $f \in \mathscr{S}_{\infty}^{\prime}\left(\mathbb{R}^{n}\right)$ such that

$$
\|f\|_{\dot{\mathcal{E}}_{p, q, u}^{s}\left(\mathbb{R}^{n}\right)}:=\sup _{Q}|Q|^{\frac{1}{u}-\frac{1}{p}}\left(\int_{Q}\left(\sum_{j \in \mathbb{Z}}\left(2^{j s}\left|\psi_{j} * f(x)\right|\right)^{p}\right)^{q / p} \mathrm{~d} x\right)^{1 / q}<+\infty,
$$

where $Q$ ranges over all cubes of dyadic edge lengths in $\mathbb{R}^{n}$.

Proof of Theorem C.3. In [26], the author introduced a more refined scale of homogeneous Triebel-Lizorkin spaces of Morrey-Lorentz type, denoted by $\dot{F}_{M_{p, q \lambda}}^{s, u}\left(\mathbb{R}^{n}\right)$. In the case $u=p=q$, those spaces coincide with the homogeneous Triebel-Lizorkin-Morrey spaces above, namely

$$
\dot{F}_{M_{p, p, \lambda}}^{s, p}\left(\mathbb{R}^{n}\right)=\dot{\mathcal{E}}_{p, p, \frac{n p}{\lambda}}^{s}\left(\mathbb{R}^{n}\right)
$$

for every $p \in(0, \infty), \lambda \in(0, n]$, and $s \in \mathbb{R}$. More precisely, their defining semi-norms are equivalent (in one case the supremum is taken over all dyadic cubes, while in the other it is taken over balls). By [26, Theorem 4.1], under condition (C.1) the space $\dot{F}_{M_{q_{2}, q_{2}, \lambda}}^{\alpha_{2}, q_{2}}\left(\mathbb{R}^{n}\right)$ embeds continuously into $\dot{F}_{M_{q_{1}, q_{1}, \lambda}}^{\alpha_{1}, q_{1}}\left(\mathbb{R}^{n}\right)$. In other words,

$$
\dot{\mathcal{E}}_{q_{2}, q_{2}, \frac{n q_{2}}{\lambda}}^{\alpha_{2}}\left(\mathbb{R}^{n}\right) \hookrightarrow \dot{\mathcal{E}}_{q_{1}, q_{1}, \frac{n q_{1}}{\lambda}}^{\alpha_{1}}\left(\mathbb{R}^{n}\right)
$$

continuously. On the other hand, [42, Theorem 1.1] tells us that

$$
\dot{\mathcal{E}}_{q_{1}, q_{1}, \frac{n q_{1}}{\lambda}}^{\alpha_{1}}\left(\mathbb{R}^{n}\right)=\dot{F}_{q_{1}, q_{1}}^{\alpha_{1} \frac{n-\lambda}{q_{1}}}\left(\mathbb{R}^{n}\right) \quad \text { and } \quad \dot{\mathcal{E}}_{q_{2}, q_{2}, \frac{n q_{2}}{\lambda}}^{\alpha_{2}}\left(\mathbb{R}^{n}\right)=\dot{F}_{q_{2}, q_{2}}^{\alpha_{2}, \frac{n-\lambda}{q_{2}}}\left(\mathbb{R}^{n}\right),
$$

with equivalent semi-norms. Finally, by [57, Theorem 3.1] we have

$$
\dot{F}_{q_{1}, q_{1}}^{\alpha_{1}, \frac{n-\lambda}{q_{1}}}\left(\mathbb{R}^{n}\right)=\mathcal{Q}_{\frac{n q_{1}}{\lambda}}^{\alpha_{1}, q_{1}}\left(\mathbb{R}^{n}\right) \quad \text { and } \quad \dot{F}_{q_{2}, q_{2}}^{\alpha_{2}, \frac{n-\lambda}{q_{2}}}\left(\mathbb{R}^{n}\right)=\mathcal{Q}_{\frac{n q_{2}}{\lambda}}^{\alpha_{2}, q_{2}}\left(\mathbb{R}^{n}\right),
$$

with equivalent semi-norms. Hence, the conclusion follows from (C.2).

We are now ready to state the important corollary of Theorem C.3 used in the proof of Theorem 4.1. Given $s \in(0,1), p \in[1, \infty)$, and an open set $\Omega \subseteq \mathbb{R}^{n}$, we recall that the Sobolev-Slobodeckij $W^{s, p}(\Omega)$-semi-norm of a measurable function $f$ is given by

$$
[f]_{W^{s, p}(\Omega)}:=\left(\iint_{\Omega \times \Omega} \frac{|f(x)-f(y)|^{p}}{|x-y|^{n+s p}} \mathrm{~d} x \mathrm{~d} y\right)^{1 / p} .
$$

Corollary C.6. Let $s \in(0,1)$ and $f \in L^{1}\left(\mathbb{R}^{n}\right)$. If

$$
\sup _{D_{r}(x) \subseteq \mathbb{R}^{n}} r^{2 s-n}[f]_{H^{s}\left(D_{r}(x)\right)}^{2}<+\infty,
$$

then,

$$
\sup _{D_{r}(x) \subseteq \mathbb{R}^{n}} r^{\frac{2 s-n}{3}}[f]_{W^{s / 3,6}\left(D_{r}(x)\right)}^{2} \leqslant C \sup _{D_{r}(x) \subseteq \mathbb{R}^{n}} r^{2 s-n}[f]_{H^{s}\left(D_{r}(x)\right)}^{2},
$$

for a constant $C=C(n, s)$. 
Proof. Since $f \in L^{1}\left(\mathbb{R}^{n}\right)$, it belongs to $\mathscr{S}^{\prime}\left(\mathbb{R}^{n}\right)$, and thus to $\mathscr{S}_{\infty}^{\prime}\left(\mathbb{R}^{n}\right)$ (see [55, Sec 5.1.2, p. 237]). Then, condition (C.4) implies that $f \in \mathcal{Q}_{n / s}^{s, 2}\left(\mathbb{R}^{n}\right)$. On the other hand, $\mathcal{Q}_{n / s}^{s, 2}\left(\mathbb{R}^{n}\right) \hookrightarrow \mathcal{Q}_{3 n / s}^{s / 3,6}\left(\mathbb{R}^{n}\right)$ continuously by Theorem C.3. Then the conclusion follows from the definition of $\mathcal{Q}_{3 n / s}^{s / 3,6}\left(\mathbb{R}^{n}\right)$ together with Remark C.2.

Acknowledgements. V.M. is supported by the Agence Nationale de la Recherche through the project ANR-14-CE25-0009-01 (MAToS). A.S. is supported by the Simons Fondation through the grant no. 579261.

\section{REFERENCES}

[1] F. Bethuel : On the singular set of stationary harmonic maps, Manuscripta Math. 78 (1993), $417-443$.

[2] L.A. Caffarelli, J.M. Roquejoffre, O. Savin : Nonlocal minimal surfaces, Comm. Pure Appl. Math. 63 (2010), 1111-1144.

[3] L.A. Caffarelli, L. Silvestre : An extension problem related to the fractional Laplacian, Comm. Partial Differential Equations 32 (2007), 1245-1260.

[4] R. Coifman, P.L. Lions, Y. Meyer, S. Semmes : Compensated compactness and Hardy spaces, J.Math. Pures Appl. 72 (1993), 247-286.

[5] L. CuI, Q. YAng : On the generalized Morrey spaces, Siberian Math. J. 46 (2005) 133-141.

[6] F. DA Lio : Compactness and bubble analysis for 1/2-harmonic maps, Ann. Inst. H. Poincaré Anal. Non Linéaire 32 (2015), 201-224.

[7] F. DA Lio : Fractional harmonic maps into manifolds in odd dimension $n>1$, Calc. Var. Partial Differential Equations 48 (2013), 421-445.

[8] F. DA Lio : Fractional harmonic maps, Recent Developments in Nonlocal Theory, De Gruyter (2018).

[9] F. DA Lio, T. Rivière : Three-term commutator estimates and the regularity of 1/2-harmonic maps into spheres, Anal. PDE 4 (2011), 149-190.

[10] F. DA Lio, T. Rivière : Sub-criticality of non-local Schrödinger systems with antisymmetric potentials and applications to half-harmonic maps, Adv. Math. 227 (2011), 1300-1348.

[11] F. Da Lio, A. Schikorra : $n / p$-harmonic maps: regularity for the sphere case, Adv. Calc. Var. 7 (2014), $1-26$.

[12] F. DA Lio, A. Schikorra : On regularity theory for $n / p$-harmonic maps into manifolds, Nonlinear Anal. 165 (2017), 182-197.

[13] F. Duzana, J.F. Grotowski : Energy minimizing harmonic maps with an obstacle at the free boundary, Manuscripta Math. 83 (1994), 291-314.

[14] F. Duzanr, J.F. Grotowski : A mixed boundary value problem for energy minimizing harmonic maps, Math. Z. 221(1996), 153-167.

[15] F. Duzana, K. Steffen : A partial regularity theorem for harmonic maps at a free boundary, Asymptotic Anal. 2 (1989), 299-343.

[16] F. Duzana, K. Steffen : An optimal estimate for the singular set of a harmonic map in the free boundary, J. Reine Angew. Math. 401 (1989), 157-187.

[17] L.C. Evans : Partial regularity for stationary harmonic maps into spheres, Arch. Ration. Mech. Anal. 116 (1991), 101-113.

[18] E.B. Fabes, C.E. Kenig, R.P. Serapioni : The local regularity of solutions of degenerate elliptic equations, Comm. Partial Differential Equations 7 (1982), 77-116.

[19] M. Giaquinta, L. Martinazzi : An introduction to the regularity theory for elliptic systems, harmonic maps and minimal graphs, Lectures Notes. Scuola Normale Superiore di Pisa 11, Edizioni della Normale, Pisa (2012).

[20] P. Grisvard : Elliptic problems in nonsmooth domains, Monographs and Studies in Mathematics, Pitman, Boston MA (1985).

[21] R. Gulliver, J. Jost : Harmonic maps which solve a free-boundary problem, J. Reine Angew. Math. 381 (1987), 61-89.

[22] R. HARdT, F.H. Lin : Partially constrained boundary conditions with energy minimizing mappings, Commun. Pure Appl. Math. 42 (1989), 309-334.

[23] J. Heinonen, T. Kilpeläinen, O. MARTio : Nonlinear potential theory of degenerate elliptic equations, Oxford Mathematical Monographs. The Clarendon Press, Oxford University Press, New York (1993).

[24] F. HÉLEIN : Régularité des applications faiblement harmoniques entre une surface et une sphère, C.R.Acad. Sci. Paris Série I 311 (1990), 519-524. 
[25] F. HÉLEIN : Régularité des applications faiblement harmoniques entre une surface et une variété riemannienne, C. R. Acad. Sci. Paris Sér. I Math. 312 (1991), 591-596.

[26] K.P. Ho : Sobolev-Jawerth embedding of Triebel-Lizorkin-Morrey-Lorentz spaces and fractional integral operator on Hardy type spaces, Math. Nachr. 287 (2014), 1674-1686.

[27] F. MAGGi : Sets of finite perimeter and geometric variational problems, An introduction to Geometric Measure Theory, Cambridge Studies in Advanced Mathematics 135, Cambridge University Press (2012).

[28] P. Mattila : Geometry of sets and measures in Euclidean spaces, Cambridge Studies in Advanced Mathematics 44, Cambridge University Press (1995).

[29] K. Mazowiecka, A. Schikorra : Fractional div-curl quantities and applications to nonlocal geometric equations, J. Funct. Anal. 275 (2018), 1-44.

[30] V. Millot, M. Pegon : Minimizing 1/2-harmonic maps into spheres, Calc. Var. and Partial Differential Equations 59 (2020), Art. 55, 37pp.

[31] V. Millot, A. Pisante : Relaxed energies for $H^{1 / 2}$-maps with values into the circle and measurable weights, Indiana Univ. Math. J. 58 (2009), 49-136.

[32] V. Millot, Y. Sire : On a fractional Ginzburg-Landau equation and 1/2-harmonic maps into spheres, Arch. Ration. Mech. Anal. 215 (2015), 125-210.

[33] V. Millot, Y. Sire, K. WANG : Asymptotics for the fractional Allen-Cahn equation and stationary nonlocal minimal surfaces, Arch. Ration. Mech. Anal. 231 (2019), 1129-1216.

[34] V. Millot, Y. Sire, H. Yu : Minimizing fractional harmonic maps on the real line in the supercritical regime, Discrete Contin. Dyn. Syst. Ser. A 38 (2018), 6195-6214.

[35] P. Mironescu, A. Pisante : A variational problem with lack of compactness for $H^{1 / 2}\left(S^{1} ; S^{1}\right)$ maps of prescribed degree, J. Funct. Anal. 217 (2004), 249-279.

[36] R. Moser : Intrinsic semiharmonic maps, J. Geom. Anal. 21 (2011), 588-598.

[37] S.A. Molchanov, E. Ostrovskit : Symmetric stable processes as traces of degenerate diffusion processes, Theory Probab. Appl. 14 (1969), 128-131.

[38] T. Rivière : Everywhere discontinuous harmonic maps into spheres, Acta Math. 175 (1995), $197-226$.

[39] T. Rivière : Conservation laws for conformally invariant variational problems, Invent. Math. 168 (2007), $1-22$.

[40] J. Roberts : A regularity theory for intrinsic minimising fractional harmonic maps, Calc. Var. Partial Differential Equations 57 (2018), Art. 109, 68pp.

[41] X. Ros-Oton, J. Serra : The Dirichlet problem for the fractional Laplacian: regularity up to the boundary, J. Math. Pures Appl. 101 (2014), 275-302.

[42] Y. Sawano, D. YAng, W. YuAn : New applications of Besov-type and Triebel-Lizorkin-type spaces, J. Math. Anal. Appl. 363 (2010), 73-85.

[43] C. Scheven : Partial regularity for stationary harmonic maps at a free boundary, Math. Z. 253 (2006), $135-157$.

[44] A. SchikorRA : Regularity of $n / 2$-harmonic maps into spheres, J. Differential Equations 252 (2012),18621911.

[45] A. SChikorra : Integro-differential harmonic maps into spheres, Comm. Partial Differential Equations 40 (2015), 506-539.

[46] A. Schikorra : $\varepsilon$-regularity for systems involving non-local, antisymmetric operators, Calc. Var. Partial Differential Equations 54 (2015), 3531-3570.

[47] R. M. Schoen : Analytic aspects of the harmonic map problem, Seminar on nonlinear partial differential equations (Berkeley, 1983), Math. Sci. Res. Inst. Publ. 2, 321-358, Springer, New York (1984).

[48] R.M. Schoen, K. Uhlenbeck : A regularity theory for harmonic maps, J. Diff. Geom. 17 (1982), $307-335$.

[49] R.M. Schoen, K. Uhlenbeck : Regularity of minimizing harmonic maps into the sphere, Invent. Math. 78 (1984), 89-100.

[50] J. Sнатан : Weak solutions and development of singularities of the $S U(2) \sigma$-model, Comm. Pure Appl. Math. 41 (1998), 459-469.

[51] W. Sickel, D. YAng, W. Yuan : Morrey and Campanato Meet Besov, Lizorkin and Triebel, Lecture Notes in Mathematics, Springer, Berlin Heidelberg (2010).

[52] L. Silvestre : Regularity of the obstacle problem for a fractional power of the Laplace operator, Comm. Pure Appl. Math. 60 (2007), 67-112.

[53] L. Simon : Theorems on regularity and singularity of energy minimizing maps, Lectures in Mathematics ETH Zürich, Birkhaüser Verlag, Basel (1996).

[54] E.M. Stein : Singular integrals and differentiability properties of functions, Princeton Mathematical Series 30, Princeton University Press, Princeton (1970).

[55] H. Triebel : Theory of function spaces, Modern Birkhäuser Classics, Springer, Basel (2010). 
[56] B.O. Turesson : Nonlinear potential theory and weighted Sobolev spaces, Lecture Notes in Mathematics 1736, Springer, Berlin (2000).

[57] D. YAng, W. YUAN : A new class of function spaces connecting Triebel-Lizorkin spaces and Q spaces, J. Funct. Anal. 255 (2008), 276-2809.

[58] W.P. ZIEMER : Weakly differentiable functions, Graduate Texts in Mathematics, Springer-Verlag, New York (1989).

Lama, Univ Paris Est Creteil, Univ Gustave Eiffel, UPem, CNRS, F-94010, Créteil, France Email address: vincent.millot@u-pec.fr

Université de Paris, Laboratoire Jacques-Louis Lions (LJLL), F-75013 Paris, France Email address: mpegon@math.univ-paris-diderot.fr

University of Pittsburgh, Department of Mathematics, 301 Thackeray Hall, Pittsburgh, PA15260, USA

Email address: armin@pitt.edu 